JOURNAL OF THE

AMERICAN MATHEMATICAL SOCIETY

Volume 25, Number 2, April 2012, Pages 355-393

S 0894-0347(2011)00723-6

Article electronically published on November 18, 2011

\title{
GRAVITATIONAL INSTANTONS FROM RATIONAL ELLIPTIC SURFACES
}

\author{
HANS-JOACHIM HEIN
}

\section{Contents}

1. Introduction 355

2. Analysis for the complex Monge-Ampère equation 360

2.1. Poincaré and Sobolev inequalities 360

2.2. Existence and decay of bounded solutions 361

3. The new gravitational instantons 366

3.1. Rational elliptic surfaces and singular fibers $\quad 366$

3.2. Ricci-flat metrics on complex torus bundles $\quad 370$

3.3. Explicit hyper-Kähler metrics near a special fiber $\quad 373$

3.4. Existence and asymptotics $\quad 380$

4. Uniqueness and moduli in the cylindrical ends case 385

4.1. Hodge theory on ALH spaces and consequences

4.2. The kernel of the linearized Einstein operator $\quad 387$

Acknowledgments $\quad 390$

References 391

\section{INTRODUCTION}

Background. This paper is motivated by questions of compactness and singularity formation in sequences of Einstein metrics on 4-manifolds. A favorite toy model in this area is the Kummer construction of hyper-Kähler metrics on K3 [5, 13]: Divide a flat complex 2-torus by the involution $x \mapsto-x$. This yields a flat orbifold with 16 singularities of type $A_{1}$. The minimal resolution of $A_{1}$ supports an explicit family of complete hyper-Kähler metrics (Eguchi-Hanson) which are uniformly asymptotic to the flat cone $\mathbb{C}^{2} / \mathbb{Z}_{2}$, and which converge to $\mathbb{C}^{2} / \mathbb{Z}_{2}$ as the area $\varepsilon$ of the exceptional divisor tends to zero. Thus, replacing neighborhoods of the $A_{1}$ points by truncated Eguchi-Hanson spaces, we obtain approximately Ricci-flat metrics on $K 3$, and there exist actual hyper-Kähler metrics close by which limit back to the orbifold as $\varepsilon \rightarrow 0$. The energy density, $|\mathrm{Rm}|^{2}$, of these metrics satisfies $\int|\mathrm{Rm}|^{2}=192 \pi^{2}$ from ChernGauß-Bonnet, and concentrates as Dirac masses around the singularities. Zooming in recovers the Eguchi-Hanson spaces as bubbles or instantons in the limit.

This picture lies at the foundation of a vast body of research on the structure of Gromov-Hausdorff limits of Einstein manifolds and other classes of metrics solving canonical elliptic equations, with particular emphasis on the 4-dimensional Einstein

Received by the editors April 24, 2010 and, in revised form, August 25, 2010, September 30, 2011, October 19, 2011, and October 23, 2011.

2010 Mathematics Subject Classification. Primary 53C25, 14J27.

(C) 2011 American Mathematical Society Reverts to public domain 28 years from publication 
case with a uniform lower volume bound [1]. In this particular case, the potential bubbles have been largely classified: A complete, simply connected, Kähler, Ricciflat 4-manifold with $\left|B\left(x_{0}, r\right)\right|>\varepsilon r^{4}$ for some $x_{0}$ and $\varepsilon>0$ and all $r \gg 1$ converges to a flat cone $\mathbb{C}^{2} / \Gamma$ with $\Gamma<\mathrm{SU}(2)$ at a rate of $r^{-4}$ [7, 52], and hence belongs to one of a number of finite-dimensional families of ALE spaces, which can be constructed algebraically [36, 37. In addition, it is widely expected that Ricci-flat ALE spaces are automatically Kähler, or equivalently hyper-Kähler.

However, if we drop the lower volume bound and thus allow for collapsing, while there do exist results concerning the limit spaces [10, 46, very little is known about the bubbling process. Hitchin [24] suggested resolving $\left(\mathbb{R}^{k} \times T^{4-k}\right) / \mathbb{Z}_{2}, k=1,2,3$, to construct instantons with $|B(r)| \sim r^{k}$, and one can imagine such spaces to form as bubbles in the Kummer construction when collapsing $T^{4} / \mathbb{Z}_{2}$ in $4-k$ directions. A folklore conjecture claims that "most" complete hyper-Kähler 4-manifolds are in fact locally asymptotic to $\mathbb{R}^{k} \times T^{4-k}$; the cases $k=3,2,1$ have been dubbed ALF, ALG, ALH. Examples in each class are known [2, 5, 11, 24, 38, 49, 53, and recent work points towards a classification of ALF spaces [5, 42, 43. However, there seems to be no clear understanding as to precisely which finiteness conditions would need to be imposed in the folklore conjecture, and rigorous examples for the appearance of ALF, ALG, or ALH spaces as singularity dilations are still lacking.

In this paper, we construct several new families of gravitational instantons that may help to shed light on some of these issues. We describe sets of ALG and ALH spaces that we expect to be exhaustive up to certain specific deformations, and two new classes of metrics with volume growth, injectivity radius decay, and curvature decay rates $r^{4 / 3}, r^{-1 / 3}, r^{-2}$, and $r^{2},(\log r)^{-1 / 2}, r^{-2}(\log r)^{-1}$, respectively. In all of these cases, the underlying complex surfaces are complements of fibers in rational elliptic surfaces, and so our metrics form one connected moduli space.

Statement of results. We need two bits of terminology for the existence result.

Definition 1.1. A rational elliptic surface is the blowup of $\mathbb{P}^{2}$ in the base points of a pencil of cubics, i.e. a family $s F+t G=0,(s: t) \in \mathbb{P}^{1}$, where $F, G$ are smooth cubics intersecting in 9 points with multiplicity. Blowing up these points, if needed repeatedly, produces an elliptic fibration $f: X \rightarrow \mathbb{P}^{1}, X=\mathbb{P}^{2} \# 9 \overline{\mathbb{P}}^{2}$.

In fact, $f=\left|-K_{X}\right|$, so for each fiber $D=f^{-1}(p)$, to be understood as a divisor, there exists precisely one rational 2 -form $\Omega$ (up to a scale) with $\operatorname{div}(\Omega)=-D$, and all rational 2-forms on $X$ arise in this way. Define $M:=X \backslash D$ and fix a small disk $\Delta=\{|z|<1\} \subset \mathbb{P}^{1}$ with $z(p)=0$ such that all fibers of $f$ over $\Delta^{*}$ are smooth.

Definition 1.2. A bad 2-cycle in $M$ is one that arises from the following process up to orientation and isotopy. Consider the topological monodromy representation of $\pi_{1}\left(\Delta^{*}\right)=\mathbb{Z}$ in the mapping class group of any fiber $F$ over $\Delta^{*}$. Take a simple loop $\gamma \subset F$ such that $[\gamma] \in H_{1}(F, \mathbb{Z})$ is indivisible and invariant under the monodromy, and move $\gamma$ around the puncture by lifting a simple loop $\bar{\gamma} \subset \Delta^{*}$ up to every point in $\gamma$ such that the union of the translates of $\gamma$ is a $T^{2}$ imbedded in $f^{-1}(\bar{\gamma})$.

While proving Proposition 3.7. we will see that $H_{2}\left(\left.X\right|_{\Delta^{*}}, \mathbb{Z}\right)$ is generated by $[F]$ and the classes of the bad cycles. Up to orientation, there are two bad cycles if the monodromy is trivial, one if the generator of the monodromy is conjugate to $\left(\begin{array}{ll}1 & b \\ 0 & 1\end{array}\right)$, $b \in \mathbb{N}$, and none at all otherwise. Moreover, bad cycles are boundaries in $X$. 
Theorem 1.3 (Existence). Let $\omega$ be any Kähler metric on $M$ such that $\int_{M} \omega^{2}<\infty$ and $\int_{C} \omega=0$ for all bad 2-cycles $C$. For instance, $\omega$ could be the restriction to $M$ of a Kähler metric on $X$. Then there exists $\alpha_{0}>0$ possibly depending on $X, \Omega, \omega$ such that for all $\alpha>\alpha_{0}$ there exists a complete Calabi-Yau metric $\omega_{\mathrm{CY}}$ on $M$ with $\omega_{\mathrm{CY}}^{2}=\alpha \Omega \wedge \bar{\Omega}$ and such that $\omega_{\mathrm{CY}}-\omega$ is d-exact on $M$.

The existence of $\omega_{\mathrm{CY}}$ follows from a method due to Tian-Yau [53, 54, for solving the Monge-Ampère equation $\left(\omega_{0}+i \partial \bar{\partial} u\right)^{2}=\alpha \Omega \wedge \bar{\Omega}$ with a complete background metric $\omega_{0}$ on $M$, which has to satisfy $\omega_{0}^{2} \approx \alpha \Omega \wedge \bar{\Omega}$ in a certain sense. We construct $\omega_{0}$ by exploiting the elliptic fibration structure in a neighborhood of $D$, modifying an idea from Gross-Wilson [20]. From a more careful analysis of $\omega_{0}$ and the PDE, one can then deduce asymptotics for $\omega_{\mathrm{CY}}=\omega_{0}+i \partial \bar{\partial} u$.

Definition 1.4. Let $\varepsilon, \delta, \ell>0, \theta \in(0,1], \tau \in \mathfrak{H} / \operatorname{PSL}(2, \mathbb{Z})$, where $\mathfrak{H}$ is the upper half-plane. Let $g_{\varepsilon, \tau}$ denote the unique flat metric of area $\varepsilon$ and modulus $\tau$ on $T^{2}$. Let $g$ be a complete Riemannian metric on an open 4-manifold $N$.

(i) The metric $g$ is called $\operatorname{ALG}(\delta,[\theta, \varepsilon, \tau])$ if there exist $r_{0}>0$, a compact subset $K \subset N$, and an imbedding $\Phi: S\left(\theta, r_{0}\right) \times T^{2} \hookrightarrow N \backslash K$ with a dense image, where $S\left(\theta, r_{0}\right):=\left\{z \in \mathbb{C}:|z|>r_{0}, 0<\arg z<2 \pi \theta\right\}$, such that

$$
\left|\nabla_{g_{\text {flat }}}^{k}\left(\Phi^{*} g-g_{\text {flat }}\right)\right|_{g_{\text {flat }}} \leq C(k)|z|^{-\delta-k}
$$

for all $k \in \mathbb{N}_{0}$, where $g_{\text {flat }}:=g_{\mathbb{C}} \oplus g_{\varepsilon, \tau}$.

(ii) The metric $g$ is called ALH if there exist $\delta>0$, a compact subset $K \subset N$, and a diffeomorphism $\Phi: \mathbb{R}^{+} \times T^{3} \rightarrow N \backslash K$ such that

$$
\left|\nabla_{g_{\text {flat }}}^{k}\left(\Phi^{*} g-g_{\text {flat }}\right)\right|_{g_{\text {flat }}} \leq C(k) e^{-\delta t}
$$

for all $k \in \mathbb{N}_{0}$, where $g_{\text {flat }}:=d t^{2} \oplus h$ for some flat metric $h$ on $T^{3}$. More specifically, we say that $g$ is $\operatorname{ALH}(\ell, \varepsilon, \tau)$ if $g$ is ALH with $h=\ell^{2} d \phi^{2} \oplus g_{\varepsilon, \tau}$ with respect to some topological splitting $T^{3}=S^{1} \times T^{2}$, with $\phi \in S^{1}=\mathbb{R} / 2 \pi \mathbb{Z}$ and $g_{\varepsilon, \tau}$ as above.

Thus, ALG spaces are asymptotic to twisted products of flat cones and flat tori, where the cones are not necessarily of the form $\mathbb{R}^{2} / \Gamma$. As for ALH spaces, the ones coming from Theorem 1.3 all have an isometrically split cross section $S^{1} \times T^{2}$, the length $2 \pi \ell$ of the circle being an intrinsic modulus of the metric. However, we will see from Theorem 1.10 that this isometric splitting can be deformed away.

The case distinction in Theorem 1.5 is according to the order of the monodromy (see Definition 1.2) as a matrix in $\operatorname{SL}(2, \mathbb{Z})$. We label the types of the singular fibers according to Kodaira's classification; cf. Section 3.1. Also, $\zeta_{3}=\exp \left(\frac{2 \pi i}{3}\right)$.

Theorem 1.5 (Asymptotics). Let $X, f, D, M, \Omega, \Delta, \omega, \alpha, \omega_{\mathrm{CY}}$ be as above and let $\varepsilon$ denote the area of the fibers of $f$ with respect to $\omega$ or $\omega_{\mathrm{CY}}$.

(i) If $D$ is smooth, then $\omega_{\mathrm{CY}}$ is $\operatorname{ALH}(\ell, \varepsilon, \tau), \ell^{2} \varepsilon=i \alpha \int_{D} R \wedge \bar{R}$, where $R$ denotes the residue of $\Omega$ along $D$, defined by $z \Omega=d z \wedge R$, and $\tau$ is the modulus of $D$.

(ii) If $D$ is singular with finite monodromy, then $\omega_{\mathrm{CY}}$ is $\operatorname{ALG}(\delta,[\theta, \varepsilon, \tau])$ with

\begin{tabular}{cccccccc}
\hline type & $\mathrm{I}_{0}^{*}$ & II & II $^{*}$ & III & III $^{*}$ & IV & IV $^{*}$ \\
$\theta$ & $\frac{1}{2}$ & $\frac{1}{6}$ & $\frac{5}{6}$ & $\frac{1}{4}$ & $\frac{3}{4}$ & $\frac{1}{3}$ & $\frac{2}{3}$ \\
$\tau$ & any & $\zeta_{3}$ & $\zeta_{3}$ & $i$ & $i$ & $\zeta_{3}$ & $\zeta_{3}$ \\
\hline
\end{tabular}

Generically, the decay rate $\delta=2$ for $\mathrm{I}_{0}^{*}, \mathrm{II}, \mathrm{III}, \mathrm{IV}$, and $\delta=\frac{4}{5}, \frac{2}{3}, \frac{1}{2}$ for $\mathrm{II}^{*}, \mathrm{III}^{*}, \mathrm{IV}^{*}$, but nongeneric ALG spaces may exhibit faster decay. 
(iii) If $D$ is singular with monodromy of infinite order, there are two possibilities. Fix any $x_{0} \in M$ and define $r(x):=1+\operatorname{dist}\left(x_{0}, x\right)$ with respect to $\omega_{\mathrm{CY}}$.

- Kodaira type $\mathrm{I}_{b}$, with monodromy conjugate to $\left(\begin{array}{cc}1 & b \\ 0 & 1\end{array}\right)$, where $b \in\{1, \ldots, 9\}$ : Then $\omega_{\mathrm{CY}}$ satisfies $\left|B\left(x_{0}, s\right)\right| \sim s^{4 / 3}$ for $s \gg 1$, $\operatorname{inj}(x) \sim|B(x, 1)| \sim r(x)^{-1 / 3}$ for all $x$, $|\mathrm{Rm}| \sim r^{-2}$, and $\left|\nabla^{k} \mathrm{Rm}\right| \lesssim_{k} r^{-2-k}$ for all $k \in \mathbb{N}$. Moreover, $\omega_{\mathrm{CY}}$ has a unique tangent cone at infinity given by the half-line, $\mathbb{R}^{+}$.

- Kodaira type $\mathrm{I}_{b}^{*}$, with monodromy conjugate to $-\left(\begin{array}{cc}1 & b \\ 0 & 1\end{array}\right)$, where $b \in\{1, \ldots, 4\}$ : In this case, $\left|B\left(x_{0}, s\right)\right| \sim s^{2}$ for $s \gg 1$, $\operatorname{inj}(x) \sim|B(x, 1)| \sim(\log r(x))^{-1 / 2}$ for all $x$, $|\mathrm{Rm}| \sim r^{-2}(\log r)^{-1}$, and $\left|\nabla^{k} \mathrm{Rm}\right| \lesssim_{k} r^{-2-k}(\log r)^{-1}$ for all $k \in \mathbb{N}$. Moreover, $\omega_{\mathrm{CY}}$ has a unique tangent cone at infinity given by $\mathbb{R}^{2} / \mathbb{Z}_{2}$.

Remark 1.6. (i) In the ALG and $\mathrm{I}_{b}^{*}$ cases, the parameter $\alpha$ from Theorem 1.3 , while corresponding to an infinitesimal Einstein deformation, probably has no geometric significance and can be changed by flowing along the vector field $r \partial_{r}$ on the tangent cone. In the ALH and $\mathrm{I}_{b}$ cases, $\alpha$ measures an intrinsic length scale of $\omega_{\mathrm{CY}}$.

(ii) Elliptic fibrations are called isotrivial if all smooth fibers are isomorphic; cf. Example 3.1 for a careful discussion of isotrivial rational elliptic surfaces. Isotrivial ALG spaces with a type $I_{0}^{*}$, II, III, or IV fiber at infinity can also be obtained from crepant resolution constructions, as done in [5, 49] and heuristically in [17, 24]. The cones on the base are then honest quotients of $\mathbb{R}^{2}$ by cyclic group actions.

(iii) The $\mathrm{I}_{b}$ manifolds appear to be the first examples of complete Ricci-flat fourmanifolds to saturate the $r^{-2}$ curvature bound from Cheeger-Tian [10].

(iv) For certain $(X, D)$ with $D$ smooth, Biquard-Minerbe [5] found cyclic groups of (anti-)automorphisms of $X$ acting freely on $M$ and isometrically with respect to $g_{\text {CY }}$ for proper choices of $\omega$. This yields asymptotically cylindrical Ricci-flat spaces with any one of the five nontrivial orientable quotients of $T^{3}$ as the cross section.

Remark 1.7. Recall that $\omega_{\mathrm{CY}}=\omega_{0}+i \partial \bar{\partial} u$, where $u$ is well-behaved and $\omega_{0}$ coincides with a Gross-Wilson style semi-flat hyper-Kähler metric near infinity.

(i) Cherkis-Kapustin 12 gave arguments to the effect that certain moduli spaces of instantons appearing in physics are rational elliptic surfaces with an $\mathrm{I}_{b}^{*}$ removed, $b \in\{0, \ldots, 4\}$, the natural $L^{2}$-metric being hyper-Kähler and semi-flat at infinity.

(ii) In the ALG and ALH cases, the deviation of $\omega_{0}$ from being flat is typically at least as large as $\omega_{\mathrm{CY}}-\omega_{0}$. However, if the elliptic fibration is isotrivial, then $\omega_{0}$ is indeed flat and we can improve $\operatorname{ALG}(\delta)$ to $\operatorname{ALG}\left(2+\frac{1}{\theta}\right)$; see also [5].

We now discuss a partial uniqueness result for ALH spaces, similar to [27.

Definition 1.8. Let $(N, g)$ be a complete Riemannian four-manifold which is ALH as in Definition 1.4(ii). We say that a tensor field $T$ on $N$ is exponentially decaying, or ED for short, if $\left|\nabla^{k} T\right| \leq C(k) e^{-\varepsilon t}$ for some $\varepsilon>0$ and all $k \in \mathbb{N}_{0}$.

Theorem 1.9 (ALH Partial Uniqueness). Let $\left(M_{i}, g_{i}\right), i=1,2$, be Ricci-flat ALH 4-manifolds with parallel orthogonal almost-complex structures $J_{i}$ and Kähler forms $\omega_{i}$. Let $\Psi: M_{1} \rightarrow M_{2}$ be a diffeomorphism such that $\Psi^{*} J_{2}=J_{1}$ and $\Psi^{*}\left[\omega_{2}\right]=\left[\omega_{1}\right]$ in de Rham cohomology. If the $M_{i}$ are diffeomorphic to the complement of a smooth fiber in a rational elliptic surface, and if $\Psi^{*} g_{2}-g_{1}=\mathrm{ED}$, then $\Psi^{*} g_{2}=g_{1}$.

What is somewhat unsatisfactory about results of this type is the condition that $\Psi^{*} g_{2}$ and $g_{1}$ be asymptotic as tensor fields. It would be desirable to prove that this automatically holds (perhaps after composing $\Psi$ with an automorphism of $\left(M_{1}, J_{1}\right)$ ) if we merely assume that the asymptotic cylinders of $\left(M_{i}, g_{i}\right)$ are isometric. 
Theorem 1.9 tells us that if we fix the leading term, then the ALH metrics from Theorem 1.3 are uniquely determined by their Kähler classes and the isomorphism type of the pencil. This amounts to a total of 24 moduli ( 8 real, 8 complex), where we ignore an overall scale. To change the leading term, we can either change $\alpha$, or move $D$ in a given pencil, thus deforming the factors in the $S^{1} \times T^{2}$ splitting $(1+2$ real moduli). We now ask whether there are any degrees of freedom left.

Theorem 1.10 (ALH Moduli). Let $M$ be an open 4-manifold diffeomorphic to the complement of a smooth fiber in a rational elliptic surface, let $g$ be an ALH hyperKähler metric on $M$, and $\mathcal{H}:=\left\{h \in C^{\infty}\left(\operatorname{Sym}^{2} T^{*} M\right): h=\right.$ parallel + ED outside a compact set\}. The kernel inside $\mathcal{H}$ of the linearization at $g$ of the Ricci curvature has dimension 29 modulo arbitrary Lie derivatives and multiples of $g$. Its subspace of ED sections has dimension 24, and the quotient is naturally isomorphic to the deformation space $\mathfrak{s l}(3, \mathbb{R}) / \mathfrak{s o}(3)$ of the flat metric on the cross section. All kernel elements are tangent to some curve of ALH hyper-Kähler metrics on $M$.

Thus, in the setting of Theorem 1.3 , besides deforming the $S^{1}$ and $T^{2}$ separately, we have 2 more moduli to break the $S^{1} \times T^{2}$ splitting. In fact, these can be realized inside every fixed Kähler class with respect to the given complex structure.

Theorems 1.9 1.10 follow from Hodge theory on manifolds with cylindrical ends, which is of course a very well-understood subject. Indeed, Kovalev 35] has sketched a general deformation theory for asymptotically cylindrical Calabi-Yau manifolds, similar to Koiso's approach [33] in the compact case. However, his parameter count is not entirely accurate, and he needs to assume an appropriate analog of the TianTodorov theorem [51, 55, which seems to be still unknown; cf. Corollary 4.3.

One way of proving the unobstructedness in Theorem 1.10 is by taking products with $T^{3}$ and appealing to Nordström's work [4] on $G_{2}$-structures. Our parameter count is consistent with [47, Proposition 3.5], though our proof provides a specific bit of extra information concerning Lie derivatives; see also Remark 4.6 .

Further questions. There are various classification issues that we intend to discuss elsewhere. Here, we would like to propose a different question: Which gravitational instantons do in fact occur as singularity models for Einstein 4-manifolds?

Problem 1.11. Let $f: X \rightarrow \mathbb{P}^{1}$ be an elliptic fibration on a $K 3$. Let $\varpi \in H^{2}(X, \mathbb{Z})$ be the Poincaré dual of the fibers and let $\varpi_{t}, t \in[0,1]$, be a smooth path in $H^{2}(X, \mathbb{R})$ with $\varpi_{t}$ inside the Kähler cone for $t<1$ and $\varpi_{1}=\varpi$. The collapsed limit metric on $\mathbb{P}^{1}$ of the Ricci-flat metrics in $\varpi_{t}$ as $t \rightarrow 1$ was identified in [20, 50, 56], cf. (3.4), fitting in with the general picture that limits of compact Einstein 4-manifolds with bounded Euler numbers are Riemannian orbifolds off a finite number of points [46]. We conjecture that exactly the following instanton bubbles appear:

- For every singular fiber of finite monodromy, an isotrivial ALG space with the dual fiber type at infinity; cf. Remark 1.6(ii), Example 3.1. and Remark 3.6(i).

- For $\mathrm{I}_{b}$, one Taub-NUT at every fixed point of the canonical $S^{1}$-action [20, 38].

- For $\mathrm{I}_{b}^{*}, b$ copies of Taub-NUT as before, plus one copy of the crepant resolution of $\left(\mathbb{R}^{3} \times S^{1}\right) / \mathbb{Z}_{2}$, as in [5], for each tail of the dual Dynkin diagram $\tilde{D}_{b+4}$.

Plan of the paper. We will apply a noncompact version, due to Tian-Yau [53, 54, of Yau's solution of the Calabi conjecture. The setup is an open complex manifold $M$ together with a complete Kähler metric $\omega_{0}$ whose Ricci form satisfies $\rho\left(\omega_{0}\right)=i \partial \bar{\partial} f$ 
with $f \rightarrow 0$ at infinity. We may then hope to find a well-behaved solution $u$ for the complex Monge-Ampère equation $\left(\omega_{0}+i \partial \bar{\partial} u\right)^{m}=e^{f} \omega_{0}^{m}$, where $m=\operatorname{dim}_{\mathbb{C}} M$.

In Section 2.2, we will cite an appropriate existence result for bounded solutions, which suffices to deduce Theorem 1.3 once $\omega_{0}$ has been constructed. However, for the proof of Theorem 1.5, we also need to show that bounded solutions converge to constants at infinity at a suitable rate. This will be done in the rest of Section 2.2, based on certain Poincaré and Sobolev inequalities from Section 2.1.

This leaves us with writing down background metrics $\omega_{0}$ on the complement of a given fiber in a rational elliptic fibration. Rather than using separation of variables as in [53, 54, which does not seem to generalize to the case of singular divisors, we focus on the elliptic fibration structure in a neighborhood of $D$, constructing $\omega_{0}$ as a Riemannian submersion with flat metrics on the fibers as in [20].

Section 3.1 provides a detailed review of rational elliptic surfaces, with examples for the isotrivial case, and of Kodaira's theory of singular fibers. Section 3.2 deals with semi-flat Calabi-Yau metrics in general. We discuss torus bundles of arbitrary fiber dimension. Section 3.3 examines the geometry of the semi-flat metrics in the elliptic surface case, extracting formulas for the periods from [29]. Section 3.4 then shows how to glue the semi-flat metrics, which are defined in a neighborhood of $D$, with given Kähler metrics on $M$ such that the theory from Section 2 applies.

Section 4 gives the proofs of Theorems 1.9 and 1.10, after briefly reviewing the necessary Hodge-theoretic machinery on asymptotically cylindrical manifolds.

Notation. Generic constants are always $>1$ and may increase within the same line. We denote metric annuli by $A(x, r, s)=B(x, s)-\bar{B}(x, r)$. Our notation for Kähler manifolds is $(M, \omega), n=\operatorname{dim}_{\mathbb{R}} M=2 m, \omega(X, Y)=\langle J X, Y\rangle, \omega=i g_{j \bar{k}} d z^{j} \wedge d \bar{z}^{k}$, so $g_{j \bar{k}}=g\left(\partial_{j}, \partial_{\bar{k}}\right)=\frac{1}{2} \delta_{j k}$ on $\mathbb{C}^{m}$. For the Ricci form, $\rho=\rho(\omega), \rho(X, Y)=\operatorname{Ric}(J X, Y)$, $[\rho]=2 \pi c_{1}(M)$, and $d^{c}:=i(\bar{\partial}-\partial), d d^{c}=2 i \partial \bar{\partial}, \Delta:=-d^{*} d=2 g^{j \bar{k}} \partial_{j} \partial_{\bar{k}}$.

\section{Analysis for the COMPlex Monge-Ampère EQuation}

2.1. Poincaré and Sobolev inequalities. $(M, g)$ denotes a Riemannian manifold of dimension $n>2$, which we assume to be complete for simplicity.

The following is from Maheux and Saloff-Coste [41, but we assume a lower Ricci bound only in a neighborbood of $B$ rather than on the whole of $M$. This is fine by inspection of their proof, which ultimately only relies on volume comparison.

Lemma 2.1 ([41, Théorème 1.1]). Let $B=B(x, r)$. If Ric $\geq-\Lambda r^{-2}$ on $10 B$ with $\Lambda \geq 0$ and if $\alpha \in\left[1, \frac{n}{n-2}\right]$, then, for all $u \in C^{\infty}(B)$, denoting $u_{B}:=f_{B} u$,

$$
\left(f_{B}\left|u-u_{B}\right|^{2 \alpha}\right)^{\frac{1}{\alpha}} \leq C(n, \Lambda) r^{2} f_{B}|\nabla u|^{2} .
$$

The second tool needed for Section 2.2 is a rough Poincaré inequality on annuli. We will prove this using the $\alpha=1$ case of (2.1), which is originally due to Buser 6 , Lemma 5.1], but we could also work with a less precise Poincaré inequality derived from the Cheeger-Colding segment inequality [8, Theorem 2.11].

Lemma 2.2. Given $r_{1}<r_{2}$ and $s$, define $A(\kappa):=A\left(x, r_{1}-\kappa s, r_{2}+\kappa s\right)$ if $r_{1}>\kappa s$. Suppose that $A:=A(0)$ is connected, Ric $\geq-C s^{-2}$ on $A(100), \sup _{y \in A(3)}|B(y, s)| \leq$ 
$C \inf _{y \in A}|B(y, s)|$, and $|A(1)| \leq N \inf _{y \in A}|B(y, s)|$. Then

$$
\int_{A}\left|u-u_{A}\right|^{2} \leq C N^{2} s^{2} \int_{A(3)}|\nabla u|^{2}
$$

for all $u \in C^{\infty}(A)$, where $u_{A}:=f_{A} u$ as before.

Proof. Pick a maximal $\frac{s}{2}$-separated set $y_{1}, \ldots, y_{m}$ in $A$. Then the balls $B_{i}=B\left(y_{i}, s\right)$ cover $A$, but the $\frac{1}{2} B_{i}$ are disjoint. By Lemma 2.1 for all $i$ and all $1 \leq \lambda \leq 10$,

$$
\int_{\lambda B_{i}}\left|u-u_{\lambda B_{i}}\right|^{2} \leq C s^{2} \int_{\lambda B_{i}}|\nabla u|^{2} .
$$

Now observe that for any $\mu \in \mathbb{R}$ we have

$$
\int_{A}\left|u-u_{A}\right|^{2} \leq \int_{A}|u-\mu|^{2} \leq 2 \sum \int_{B_{i}}\left|u-u_{B_{i}}\right|^{2}+2\left(\max \left|B_{i}\right|\right) \sum\left|u_{B_{i}}-\mu\right|^{2} .
$$

We estimate the first sum by using (2.3) with $\lambda=1$, plus the fact that the covering $\left\{B_{i}\right\}$ has multiplicity at most $C$. For the second sum, fix $\mu$ to be the average of the $u_{B_{i}}, a_{i}:=u_{B_{i}}-\mu$, and note that $\sum a_{i}^{2}=\frac{1}{2 m} \sum\left|a_{i}-a_{j}\right|^{2}$ because $\sum a_{i}=0$. Then, for every $(i, j)$, connect $B_{i}$ and $B_{j}$ by a ball chain without loops, expand $a_{i}-a_{j}$ as a telescoping sum along that chain, and rearrange to obtain

$$
\sum\left|u_{B_{i}}-\mu\right|^{2} \leq C N^{2} \sum_{i} \sum_{B_{i} \cap B_{k} \neq \emptyset}\left|u_{B_{i}}-u_{B_{k}}\right|^{2} .
$$

Next, for any constant $\nu \in \mathbb{R}$, by Cauchy-Schwarz,

$$
\begin{aligned}
\left|u_{B_{i}}-u_{B_{k}}\right|^{2} & \leq \frac{1}{\left|B_{i}\right|\left|B_{k}\right|} \int_{B_{i} \times B_{k}}|u(x)-u(y)|^{2} d x d y \\
& \leq 4 \frac{\left|B_{i} \cup B_{k}\right|}{\left|B_{i}\right|\left|B_{k}\right|} \int_{B_{i} \cup B_{k}}|u(x)-\nu|^{2} d x .
\end{aligned}
$$

Now $B_{i} \cup B_{k} \subset 3 B_{i}$ since $B_{i} \cap B_{k} \neq \emptyset$, so put $\nu:=u_{3 B_{i}}$, apply (2.3) for $\lambda=3$, and use that the covering $\left\{3 B_{i}\right\}$ still has multiplicity bounded by $C$.

The method employed in the proof of Lemma 2.2 is due to Grigoryan and SaloffCoste [19] in much greater generality. As a corollary of the proof, it is worth noting that $N$ in (2.2) could be replaced by the largest number of balls in a shortest ball chain connecting any two points in $A$. In particular, this then recovers the optimal Neumann-Poincaré constant $r^{2}$ for annuli of the form $A(r, r+1)$ in $\mathbb{R}^{n}$ up to some bounded factor. Recall that the Dirichlet-Poincaré constant of $A(r, r+1)$ in $\mathbb{R}^{n}$ is uniformly bounded; this distinction is useful to keep in mind for Section 2.2.

2.2. Existence and decay of bounded solutions. We first quote an existence result for uniformly bounded solutions to the complex Monge-Ampère equation on certain complete noncompact Kähler manifolds. Tian-Yau [53, Theorem 1.1] would be sufficient for our purposes, but we will use a somewhat more precise result from [22, 23], which in particular makes it explicit that the solutions obtained in [53] are of finite Dirichlet energy. To state this result, we first require a definition.

Definition 2.3. $(M, g)$ is called $\operatorname{SOB}(\beta)$ if there exist $x_{0} \in M$ and $C \geq 1$ such that $A\left(x_{0}, r, r+s\right)$ is connected for all $r \geq C, s>0,\left|B\left(x_{0}, s\right)\right| \leq C s^{\beta}$ for all $s \geq C$, and $\left|B\left(x,\left(1-\frac{1}{C}\right) r(x)\right)\right| \geq \frac{1}{C} r(x)^{\beta}$ and $\operatorname{Ric}(x) \geq-C r(x)^{-2}$ if $r(x):=\operatorname{dist}\left(x_{0}, x\right) \geq C$. 
If a manifold satisfies condition $\operatorname{SOB}(\beta)$, then it admits certain natural weighted global Sobolev inequalities; see 23 . Then let $\left(M^{m}, \omega_{0}\right)$ denote a complete Kähler manifold with $|\mathrm{Rm}|+|\nabla \mathrm{Scal}|+\left|\nabla^{2} \mathrm{Scal}\right| \leq C$ such that $\operatorname{SOB}(\beta)$ holds.

Proposition 2.4. Let $f \in C^{2, \alpha}(M)$ satisfy $|f| \leq C r^{-\mu}$ for some $\mu>2$. If $\beta \leq 2$, then assume in addition that $\int\left(e^{f}-1\right) \omega_{0}^{m}=0$. Then there exists an $\bar{\alpha} \in(0, \alpha]$ and $u \in C^{4, \bar{\alpha}}(M)$ such that $\left(\omega_{0}+i \partial \bar{\partial} u\right)^{m}=e^{f} \omega_{0}^{m}$. If $\beta \leq 2$, then $\int|\nabla u|^{2} \omega_{0}^{m}<\infty$.

The Hölder spaces are to be understood with uniform global bounds on $M$, but we of course also get higher local regularity from standard elliptic theory.

From Li-Tam [39], $\operatorname{SOB}(\beta)$ Riemannian manifolds are nonparabolic if and only if $\beta>2$. Yau's method for solving the Monge-Ampère equation produces bounded solutions by default. Thus, if $\beta \leq 2$, the integrability condition $\int\left(e^{f}-1\right) \omega_{0}^{m}=0$ is necessary; intuitively, it cancels out the monopole term in the multipole expansion of $u$, which should be comparable to the Green's function, hence unbounded in this case. In our main application (Theorem[1.3),$f=\log \left((\alpha \Omega \wedge \bar{\Omega}) / \omega_{0}^{2}\right) \in C_{0}^{\infty}(M)$, but indeed $\beta \leq 2$. We will satisfy the integrability condition by replacing $\omega_{0}$ by $\omega_{0}+\gamma$ if necessary, where $\gamma$ is explicit, compactly supported, and in fact $\gamma=i \partial \bar{\partial} \phi$, where $\phi$ is indeed comparable to a Green's function on $M$; cf. Remark 3.8(i).

We now ask whether the solutions from Proposition 2.4 converge to constants at infinity, and if so, then at what rate. Proposition 2.9 collects what we need in this direction in order to prove the asymptotics in Theorem 1.5.

The main underlying idea works for $\beta \leq 2$ only and strongly relies on the fact that $\int|\nabla u|^{2}<\infty$ in this case. We write $M$ as a union of annuli $A_{i}=A\left(x_{0}, r_{i}, r_{i+1}\right)$ and use integration by parts to deduce $\int_{A_{i}}|\nabla u|^{2} \leq C e^{-\delta i}$ for some $\delta=\delta(u, f)>0$. The key condition needed to make this work is that the $A_{i}$ have their first Neumann eigenvalues bounded below by $\frac{1}{C}\left(r_{i+1}-r_{i}\right)^{-2}$. There are two different assumptions under which we are able to prove (by using Lemmas 2.12.2) that this is indeed the case: (1) $A_{i} \subset B\left(x_{i}, \rho_{i}\right)$ with $x_{i} \in A_{i}, \rho_{i} \sim r_{i+1}-r_{i}=o\left(r_{i}\right)$, and (2) $r_{i+1}-r_{i} \sim r_{i}$. Definition 2.5 gives the precise conditions needed for (1); the resulting bounds for the integral of $|\nabla u|^{2}$ over $B(x, 1)$ decay almost exponentially in terms of $r(x)$. For $(2)$, it suffices to assume $\operatorname{SOB}(\beta)$, but we only get polynomial energy decay.

In each case, if $|B(x, 1)|$ does not decay too fast, we then apply Moser iteration over $B(x, 1)$ to get $L^{\infty}$ decay, based on Lemma 2.1 with $\alpha>1$. Derivative bounds follow from Schauder theory, in the setting of Definitions 2.6 and 2.8 .

Definition 2.5. A complete Riemannian manifold $M$ with one end will be called $\operatorname{CYL}(\gamma)$ with $\gamma \in[0,1)$, indicating "cylindrical" asymptotic behavior, if there exist $x_{0} \in M$ and $C \geq 1$ such that $\left|B\left(x_{0}, s\right)\right| \leq C s^{2}$ for all $s \geq C$, and

$$
A\left(x_{0}, r(x)-\frac{1}{C} r(x)^{\gamma}, r(x)+\frac{1}{C} r(x)^{\gamma}\right) \subset B\left(x, C r(x)^{\gamma}\right)
$$

as well as $\operatorname{Ric}(x) \geq-C r(x)^{-2 \gamma}$ for all $x \in M$ with $r(x) \geq C$.

Definition 2.6. Let $\left(M^{n}, g\right)$ again be a complete Riemannian $n$-manifold, and let $B \subset \mathbb{R}^{n}$ denote the unit ball with respect to the Euclidean metric $g_{0}$.

(i) We say that $M$ is $\operatorname{HMG}(\lambda, k, \alpha)$ with $\lambda \in[0,1], k \in \mathbb{N}_{0}, \alpha \in(0,1)$ (to be read as " $C^{k, \alpha}$ homogeneous at scales $r^{\lambda "}$ ) if there exist $x_{0} \in M$ and $C \geq 1$ such that if $x \in M$ and $r(x) \geq C$, there exists a smooth map $\Phi_{x}: B \rightarrow M$ with maximal rank, $\Phi_{x}(0)=x$, and $\Phi_{x}(B) \supset B\left(x, \frac{1}{C} r(x)^{\lambda}\right)$, such that $h:=r(x)^{-2 \lambda} \Phi_{x}^{*} g$ satisfies

$$
\frac{1}{C} g_{0} \leq h \leq C g_{0},\left\|h-g_{0}\right\|_{C^{k, \alpha}\left(B, g_{0}\right)} \leq C .
$$


(ii) If $M$ is $\operatorname{HMG}(\lambda, k, \alpha)$, the following defines weighted Hölder seminorms for functions: For any smooth weight $\phi:[1, \infty) \rightarrow[1, \infty)$ and $l \in \mathbb{N}_{0}, \gamma \in(0,1)$, put

$$
[u]_{\phi, l, \gamma}:=\sup \left\{\phi(r(x))\left\|u \circ \Phi_{x}\right\|_{C^{l, \gamma}\left(B, g_{0}\right)}: r(x) \geq C\right\} .
$$

We then have a simple criterion for homogeneity in terms of curvature decay; we omit the proof because this is almost identical to [53, Proposition 1.2].

Lemma 2.7. A complete Kähler manifold with $|\mathrm{Rm}|+\sum_{i=1}^{k-1} r^{i \lambda}\left|\nabla^{i} \mathrm{Scal}\right| \leq C r^{-2 \lambda}$ for some $k \in \mathbb{N}$ and $\lambda \in[0,1]$ is $\operatorname{HMG}(\lambda, k, \alpha)$ for every $\alpha \in(0,1)$. Moreover, the maps $\Phi_{x}: \mathbb{C}^{m} \supset B \rightarrow M$ in Definition 2.6 can be taken to be holomorphic.

Our final definition specifies a notion of diameter growth.

Definition 2.8. A complete Riemannian manifold $M$ has at most linear diameter growth if there exist $x_{0} \in M$ and $C \geq 1$ such that for every $s \geq C$ and $x_{1}, x_{2} \in M$ with $r\left(x_{1}\right)=r\left(x_{2}\right)=s$, there exists a curve $\gamma$ of length at most $C s$ connecting $x_{1}$ and $x_{2}$ in $M$ such that $r \circ \gamma \geq s$.

With these preliminaries out of the way, we can state and prove our main result concerning the decay of bounded solutions, as sketched above.

Proposition 2.9. Let $\left(M^{m}, \omega_{0}\right)$ be a complete Kähler manifold. Let u, $f$ be smooth functions on $M$ such that $\sup \left|\nabla^{i} u\right|+\sup \left|\nabla^{i} f\right|<\infty$ for all $i \in \mathbb{N}_{0}$ and

$$
\left(\omega_{0}+i \partial \bar{\partial} u\right)^{m}=e^{f} \omega_{0}^{m} .
$$

(ia) Assume that $M$ is $\operatorname{CYL}(\gamma)$ with $\gamma \in[0,1)$ and $\exp \left(\kappa r(x)^{1-\gamma}\right)|B(x, 1)| \rightarrow \infty$ as $r(x) \rightarrow \infty$ for every fixed $\kappa>0$. If there exists $\varepsilon>0$ such that

$$
\int_{M}|\nabla u|^{2} \omega_{0}^{m}<\infty, \quad|f| \leq C \exp \left(-\varepsilon r^{1-\gamma}\right),
$$

then there exists $\delta>0$ such that for all $x \in M$,

$$
\sup _{B(x, 1)}\left|u-u_{B(x, 1)}\right| \leq C \exp \left(-\delta r(x)^{1-\gamma}\right) .
$$

(ib) Assume that $M$ satisfies $\operatorname{SOB}(\beta)$ with $\beta \in(0,2]$ and $r(x)^{\kappa}|B(x, 1)| \rightarrow \infty$ as $r(x) \rightarrow \infty$ for every fixed $\kappa>0$. If there exists $\varepsilon>0$ such that

$$
\int_{M}|\nabla u|^{2} \omega_{0}^{m}<\infty, \quad|f| \leq C r^{-\beta-\varepsilon},
$$

then there exists $\delta>0$ such that for all $x \in M$,

$$
\sup _{B(x, 1)}\left|u-u_{B(x, 1)}\right| \leq C r(x)^{-\delta} .
$$

(ii) Let $M$ be $\operatorname{HMG}(\lambda, k, \alpha)$, with all $\Phi_{x}$ holomorphic, of at most linear diameter growth. Let $\phi:[1, \infty) \rightarrow[1, \infty)$ be a smooth function with $\phi\left(t-\frac{1}{2} t^{\lambda}\right) \geq \frac{1}{C} \phi(t)$ for all $t \geq C$ and such that $t^{-\delta} \phi(t)$ is nondecreasing for some $\delta>0$. If

$$
\sup _{x \in M} r(x)^{1-\lambda} \phi(r(x)) \sup _{B(x, 1)}\left|u-u_{B(x, 1)}\right|<\infty, \quad[f]_{\psi, k, \alpha}<\infty,
$$

where $\psi(t):=t^{1-\lambda} \phi(t)$, then we have

$$
[u-\bar{u}]_{\phi, k+2, \alpha}<\infty
$$

for some constant $\bar{u} \in \mathbb{R}$. 
Proof. (i) Define $\omega:=\omega_{0}+i \partial \bar{\partial} u$ and $T:=\sum_{k=0}^{m-1}\left(\omega_{0}^{k} \wedge \omega^{m-1-k}\right)$, so $\omega^{m}-\omega_{0}^{m}=$ $\frac{1}{2} d d^{c} u \wedge T$. For $\zeta \in C_{0}^{\infty}(M), \mu \in \mathbb{R}$, multiply by $\zeta(u-\mu)$ and integrate by parts:

$$
\int \zeta|\nabla u|^{2} \omega_{0}^{m} \leq-m\left[\int(u-\mu) d \zeta \wedge d^{c} u \wedge T+2 \int \zeta(u-\mu)\left(e^{f}-1\right) \omega_{0}^{m}\right] .
$$

Given a sequence $C \leq r_{1}<r_{2}<\ldots$, define annuli $A_{i}:=\left\{r_{i} \leq r \leq r_{i+1}\right\}$ and ends $E_{i}:=\left\{r \geq r_{i}\right\}$. Construct cutoff functions $\zeta_{i}$ with $\zeta_{i} \equiv 1$ on $E_{i+1}, \operatorname{supp}\left(\zeta_{i}\right) \subset E_{i}$, and $\left(r_{i+1}-r_{i}\right)\left|\nabla \zeta_{i}\right| \leq C$, uniformly in $i$. Fix a bump function $\chi$ with $\chi(t)=1$ for $t<1$ and $\chi(t)=0$ for $t>2$, apply (2.4) with $\zeta:=\left(\chi \circ \frac{r}{R}\right) \zeta_{i}$, and let $R \rightarrow \infty$. The term with $\chi^{\prime}$ disappears by Cauchy-Schwarz since $|u| \leq C, \omega \sim \omega_{0}, \int|\nabla u|^{2}<\infty$, and $\left|B\left(x_{0}, s\right)\right| \leq C s^{\beta}(\beta \leq 2)$. Thus, if $|\mu| \leq C$, then, integrating against $\omega_{0}^{m}$,

$$
\begin{aligned}
& \int_{E_{i+1}}|\nabla u|^{2} \leq \int \zeta_{i}|\nabla u|^{2} \leq C \int\left|\nabla \zeta_{i}\right||u-\mu \| \nabla u|+C \int \zeta_{i}|u-\mu||f| \\
& \leq C\left(r_{i+1}-r_{i}\right)^{-1}\|u-\mu\|_{L^{2}\left(A_{i}\right)}\|\nabla u\|_{L^{2}\left(A_{i}\right)}+C \sum_{j=i}^{\infty} r_{j+1}^{\beta}\|f\|_{L^{\infty}\left(A_{j}\right)} .
\end{aligned}
$$

We can now explain the main idea: If $\|u-\mu\|_{L^{2}\left(A_{i}\right)} \leq C\left(r_{i+1}-r_{i}\right)\|\nabla u\|_{L^{2}\left(A_{i}\right)}$ and if $f \in C_{0}^{\infty}(M)$, then (2.5) implies that $\|\nabla u\|_{L^{2}\left(E_{i}\right)} \leq C \exp (-\delta i)$ by iteration, for some small $\delta=\delta(u, f)>0$. In the settings in (ia) and (ib), such a Poincaré bound is essentially always available, given an appropriate choice for $\left\{r_{i}\right\}$.

(ia) Pick $x_{i} \in A_{i}$ such that $\rho_{i}:=r\left(x_{i}\right)=\frac{1}{2}\left(r_{i}+r_{i+1}\right)$. Then, by assumption,

$$
A_{i}^{\prime}:=A\left(x_{0}, \rho_{i}-\frac{1}{C} \rho_{i}^{\gamma}, \rho_{i}+\frac{1}{C} \rho_{i}^{\gamma}\right) \subset B_{i}:=B\left(x_{i}, C \rho_{i}^{\gamma}\right) .
$$

Suppose the $r_{i}$ are such that $A_{i} \subset A_{i}^{\prime}$ and $B_{i} \subset A_{i}^{\prime \prime}:=A_{i-K} \cup A_{i-K+1} \cup \ldots \cup A_{i+K}$ for some $K \in \mathbb{N}, K \leq C$, and all $i \geq C$. If $\mu:=u_{B_{i}}$, then Lemma 2.1 yields

$$
\int_{A_{i}}|u-\mu|^{2} \leq \int_{B_{i}}|u-\mu|^{2} \leq C \rho_{i}^{2 \gamma} \int_{B_{i}}|\nabla u|^{2} \leq C \rho_{i}^{2 \gamma} \int_{A_{i}^{\prime \prime}}|\nabla u|^{2} .
$$

The essentially unique choice of radii which makes this work is $r_{i}:=\frac{1}{C} i^{\frac{1}{1-\gamma}}$. Define $Q_{i}:=\int_{E_{i}}|\nabla u|^{2}$. Then (2.5) and (2.6) imply that for all $i \geq C$,

$$
Q_{i+K} \leq Q_{i+1} \leq C\left(Q_{i-K}-Q_{i+K}\right)+C e^{-\frac{\varepsilon}{C} i},
$$

since $\rho_{i}^{\gamma} \leq C\left(r_{i+1}-r_{i}\right)$, and by the decay assumption for $f$. Hence, $Q_{2 K i} \leq C e^{-\delta i}$ for some $\delta>0$ by induction, and in particular, for all $x \in M$,

$$
\int_{B(x, 1)}|\nabla u|^{2} \leq C \exp \left(-\delta r(x)^{1-\gamma}\right) .
$$

Using Lemma 2.1 for $\alpha>1$ and the assumed lower bound on the decay of $|B(x, 1)|$ as $r(x) \rightarrow \infty$, we can now apply Moser iteration over $B(x, 1)$ to obtain the result.

(ib) The argument here is entirely analogous: If $r_{i}=2^{i} C$ and $\mu=u_{A_{i}}$, then we can replace (2.6) by Lemma 2.2, setting $s=\frac{1}{C} r_{i}$ there, and make $K=1$.

(ii) As in Kovalev [34, we define a linear differential operator $L_{u}$ by setting

$$
L_{u}(v) \omega_{0}^{m}:=i \partial \bar{\partial} v \wedge \sum_{k=0}^{m-1} \omega_{0}^{k} \wedge\left(\omega_{0}+i \partial \bar{\partial} u\right)^{m-1-k} .
$$


For any $x \in M$, put $u_{x}:=u-u_{B(x, 1)}$. Then $L_{u}\left(u_{x}\right)=e^{f}-1$. Now, for any given $\mu \in[0,1]$, we can consider the following statement, $S(\mu)$ :

$$
r(x) \geq C \Longrightarrow\left\|u_{x}\right\|_{C^{k+2, \alpha}\left(B\left(x, \frac{1}{C} r(x)^{\mu}\right), r(x)^{-2 \mu} \omega_{0}\right)} \leq C \phi(r(x))^{-1} r(x)^{\lambda-1} .
$$

Claim. $S(0)$ is true, and $S(\mu)$ implies $S(\nu)$ with $\nu:=\min \left\{\lambda, \mu+\frac{\delta}{k+2+\alpha}\right\}$.

Thus, $S(\lambda)$ is true, so $|\nabla u| \leq C(r \phi(r))^{-1}$ and hence $|u-\bar{u}| \leq C \phi(r)^{-1}$ for some constant $\bar{u} \in \mathbb{R}$, by our assumptions on $\phi$ and on the diameter growth of $M$. If we use $S(\lambda)$ again, this then proves (ii), and in fact slightly more if $\lambda<1$.

Proof of the claim. Given any $\kappa \in[0, \lambda]$, we define

$$
B_{x}(\kappa):=\Phi_{x}^{-1}\left(B\left(x, \frac{1}{C} r(x)^{\kappa}\right)\right), \omega_{x}(\kappa):=r(x)^{-2 \kappa} \Phi_{x}^{*} \omega_{0} .
$$

To show that $S(0)$ is true, we pull the equation $L_{u}\left(u_{x}\right)=e^{f}-1$ back by $\Phi_{x}$ and apply Schauder theory on $B_{x}(0)$, using the $\omega_{x}(0)$-Hölder norms. Notice that

$$
\left\|f \circ \Phi_{x}\right\|_{C^{k, \alpha}\left(B_{x}(0), \omega_{x}(0)\right)} \leq C \phi(r(x))^{-1} r(x)^{\lambda-1}
$$

because $[f]_{\psi, k, \alpha}<\infty$, which yields a suitable $C^{k, \alpha}$ bound on the larger ball $B_{x}(\lambda)$ with respect to the stronger $\omega_{x}(\lambda)$-norms. The coefficients of $L_{u}$ are appropriately bounded because $u$ has bounded derivatives by assumption, and Schauder theory holds with a uniform constant because of $\operatorname{HMG}(\lambda, k, \alpha)$, restricted to $B_{x}(0)$.

Now assume $S(\mu)$ and let $\nu$ be as given. We wish to apply Schauder theory with respect to the $\omega_{x}(\nu)$-Hölder norms on $B_{x}(\mu)$. The key point is that the coefficients of $L_{u}$ are indeed bounded in $C^{k, \alpha}\left(B_{x}(\mu), \omega_{x}(\nu)\right)$; this follows from $S(\mu), \phi(t) \geq t^{\delta}$, and $(k+2+\alpha) \nu \leq(k+2+\alpha) \mu+\delta$. We therefore obtain

$$
\left\|u_{x} \circ \Phi_{x}\right\|_{C^{k+2, \alpha}\left(B_{x}(\mu), \omega_{x}(\nu)\right)} \leq C \phi(r(x))^{-1} r(x)^{\lambda-1} .
$$

It remains to extend this from $B_{x}(\mu)$ to $B_{x}(\nu)$. But (2.7) still holds with $x$ replaced by any $y \in B\left(x, \frac{1}{C} r(x)^{\nu}\right)$, and $u_{x}, u_{y}$ differ by a constant. With $\phi\left(t-\frac{1}{2} t^{\lambda}\right) \geq \frac{1}{C} \phi(t)$, the derivative bounds therefore extend trivially. To then also extend the pointwise bound, we simply integrate the gradient bound along $\omega_{0}$-geodesics in $M$.

Example 2.10. Consider the background manifolds $\left(M, \omega_{0}\right)$ used in constructing the $\mathrm{I}_{b}$ spaces in Theorem 1.5(iii). We will see in Section 3.3 that these are $\operatorname{SOB}\left(\frac{4}{3}\right)$ and $\operatorname{CYL}\left(\frac{1}{3}\right)$, with $|B(x, 1)|$ decaying only polynomially. The relevant $f \in C_{0}^{\infty}(M)$. Proposition 2.9 gives $|u-\bar{u}| \leq C \exp \left(-\delta r^{-2 / 3}\right)$ with all derivatives. Moreover, it is easy to write down bounded harmonic functions outside a compact set that behave exactly like this, except for the precise value of $\delta$; see Remark 3.8.(ii).

On ALG spaces with cone angle $2 \pi \theta$, Proposition 2.9 yields $|u-\bar{u}| \leq C r^{-\delta}$ for some $\delta>0$, with an improvement of $r^{-1}$ for each derivative. Once this much decay is known, one can then use linear analysis to push $\delta$ all the way up to $\frac{1}{\theta}$.

We conclude here by briefly putting Proposition 2.9 into context. The solutions constructed in [53, 54] are uniformly bounded, along with all their derivatives, but do not a priori enjoy any decay properties whatsoever. On the other hand, once one proves some decay, one can then employ linear methods to optimize the rate.

We wish to emphasize that the analysis needed to establish any decay at all is of necessity quite different depending on whether $\beta \leq 2$ or $\beta>2$ (in 34, an estimate for the $\beta=2 m$ case from [54] was erroneously applied in a situation where $\beta=1$ ). Recall that the solutions in [53, 54] are obtained as limits of solutions $u_{\varepsilon}$ to certain 
relaxed problems, where $u_{\varepsilon}$ goes to zero rapidly at infinity, but not uniformly in $\varepsilon$. If $\beta>2$, there are then various ways of proving (weaker but) uniform decay for $u_{\varepsilon}$, e.g. by weighted Moser iteration [23, 27], or via the barrier method [49, 54].

To illustrate the barrier method, suppose that $\beta>2$ is an integer and $\left(M, \omega_{0}\right)$ is asymptotically a bundle with bounded fibers over a $\beta$-dimensional cone with radius function $r$. Then $\Delta\left(r^{2-\mu}\right) \approx(2-\mu)(\beta-\mu) r^{-\mu}<0$ if $\mu \in(2, \beta)$, so $\left|u_{\varepsilon}\right| \leq C_{\mu} r^{2-\mu}$ for every $\mu<\beta$ if $f \in C_{0}^{\infty}(M)$. Here, we have used the maximum principle for the complex Monge-Ampère operator $\mathcal{M}$, the fact that $\mathcal{M}\left(r^{2-\mu}\right) \approx \Delta\left(r^{2-\mu}\right)$, a uniform bound $\left|u_{\varepsilon}\right| \leq C$ [23, 54, and a nonuniform bound $u_{\varepsilon}=o_{\varepsilon}(1)$ as $r \rightarrow \infty$ [53].

While the present paper was under review, I learned of a recent paper by KöhlerKühnel [32, which proves $u-\bar{u}=O\left(r^{-N}\right)$ for all $N$ for the Calabi-Yau manifolds in 53 . Their idea is to get some polynomial initial decay from a weighted version of Yau's $C^{2}$-estimate, and then to use separation of variables to improve this. The metric $\omega_{0}$ is $\operatorname{SOB}\left(\frac{2 m}{m+1}\right)$ and $\operatorname{CYL}\left(\frac{1}{m+1}\right)$ in their case, so $u-\bar{u}=O\left(\exp \left(-\delta r^{\frac{m}{m+1}}\right)\right)$ by Proposition 2.9, which can likely be combined with 32 to obtain a sharp bound $u-\bar{u}=O\left(r^{-\alpha} \exp \left(-\delta_{0} r^{\frac{m}{m+1}}\right)\right)$, where $\alpha=\frac{m-2}{2(m+1)}$ and $\delta_{0}$ is spectral.

\section{The NEW GRAVitational instantons}

This section leads up to the proof of Theorems 1.3 and 1.5 in Section 3.4.

Section 3.1 describes the basic properties of rational elliptic surfaces and works out some isotrivial examples. We also summarize Kodaira's results from 29] on the structure of singular fibers in elliptic fibrations in general.

Section 3.2 describes a general construction of hyper-Kähler metrics on the total spaces of polarized families of complex tori. These metrics will be semi-flat, i.e. flat when restricted to any fiber. This is a variation on a classical theme, and we clarify some relations between similar constructions in the literature.

Section 3.3 studies relatively minimal elliptic fibrations $f: U \rightarrow \Delta$ that admit a section. Each such $f$ is isomorphic to an explicit normal form that can be extracted from 29. Given a rational 2 -form $\Omega$ on $U$ such that $\operatorname{div}(\Omega)$ is an integer multiple of $D=f^{-1}(0)$, this allows us to write down explicit semi-flat hyper-Kähler metrics with volume form $\Omega \wedge \bar{\Omega}$ on $U \backslash D$, and read off their geometry. The relevant cases are $\operatorname{div}(\Omega)=0$, as in $[20$, and $\operatorname{div}(\Omega)=-D$, as on a rational elliptic surface.

Given a rational elliptic surface $f: X \rightarrow \mathbb{P}^{1}$, a fiber $D=f^{-1}(p)$, and a rational 2 -form $\Omega$ with $\operatorname{div}(\Omega)=-D$, we thus obtain explicit hyper-Kähler metrics $\omega_{\mathrm{sf}, \varepsilon}$ on $U \backslash D$ with volume form $\Omega \wedge \bar{\Omega}$ such that the fibers have area $\varepsilon$ and $D$ is at infinite distance. We then need to glue up $\omega_{\mathrm{sf}, \varepsilon}$ with given Kähler metrics on $X \backslash U$.

3.1. Rational elliptic surfaces and singular fibers. Our references are [3, 15, 21, 29, 44, 45. Much of what we say here ought to be standard, or at least folklore, in algebraic geometry. However, we are not aware of any exposition of the theory of elliptic surfaces that provides the amount of detail needed for our construction of gravitational instantons, not even for the rather basic Example 3.1 below.

Consider a rational elliptic surface $X$ as in Definition 1.1. The natural fibration $f: X \rightarrow \mathbb{P}^{1}$ has an intrinsic description as the complete linear system $\left|-K_{X}\right|$, so for each fiber $D=f^{-1}(p)$ there exists a rational 2 -form $\Omega$ on $X$, which is unique up to a scale, such that $\operatorname{div}(\Omega)=-D$, and all rational 2 -forms on $X$ are obtained in this way. Moreover, $f$ is the only elliptic fibration on $X$. When blowing up a base point until all tangencies are resolved, the last exceptional divisor, which is a (-1)-curve, 
becomes a global section of $f$. All fibers of $f$ are reduced; i.e., the multiplicities of $f$ along the irreducible components of any fiber are coprime. A fiber $f^{-1}(p)$ in a general elliptic fibration is reduced if and only if $f$ admits a section near $p$.

Rational elliptic surfaces are characterized as those smooth projective surfaces that admit a rational 2 -form $\Omega$ and an elliptic fibration whose fibers do not contain $(-1)$-curves such that $\operatorname{div}(\Omega)$ is an integer multiple of a fiber. This basically shows that we do not have any other examples to work with in our construction.

As a last general point, notice that the space of cubics in $\mathbb{P}^{2}$ is a $\mathbb{P}^{9}$, so a general set of 9 points in $\mathbb{P}^{2}$ determines a unique cubic passing through it, and the space of 9-point subsets of $\mathbb{P}^{2}$ modulo PGL(3) has dimension $9 \cdot 2-8=10$. On the other hand, if cubics $F$ and $G$ intersect in finitely many points, and $H$ is another cubic passing through these, then, by the $A F+B G$ theorem, $H$ must already be a linear combination of $F$ and $G$. Thus, again mod PGL(3), 9-point sets with more than one cubic passing through them depend on $2 \cdot(9-1)-8=8$ free parameters only. If we blow up $\mathbb{P}^{2}$ in a 9 -point set that does not determine a pencil of cubics, then the resulting $X$ still has rational 2 -forms $\Omega$, and sometimes an elliptic fibration as well, but then one fiber $D$ has to have multiplicity $m>1$, and $\operatorname{div}(\Omega)$ is either $-\frac{1}{m} D$ or disconnected, so our construction of gravitational instantons breaks down.

Example 3.1. Let $E=\mathbb{C} / \Lambda$ be a complex torus and let $\Gamma$ be a finite subgroup of $\operatorname{Aut}(E)$, so $\Gamma$ is generated by a root of unity and either $\Gamma=\mathbb{Z}_{2}$ (for any $\Lambda$ ), $\Gamma=\mathbb{Z}_{4}$ (for $\Lambda=\mathbb{Z}[i])$, or $\Gamma=\mathbb{Z}_{3}, \mathbb{Z}_{6}\left(\right.$ for $\left.\Lambda=\mathbb{Z}\left[\zeta_{3}\right]\right), \zeta_{m}:=\exp (2 \pi i / m)$. Then $\Gamma$ acts on $\mathbb{P}^{1}$ in a natural way, and the minimal resolution $X$ of the singular surface $\left(\mathbb{P}^{1} \times E\right) / \Gamma$ comes with an obvious elliptic fibration $f: X \rightarrow \mathbb{P}^{1}$ with two singular fibers and all smooth fibers isomorphic to $E$. Blowing down all (-1)-curves in the singular fibers makes $X$ rational elliptic. Below, we work this out explicitly for $\Gamma=\mathbb{Z}_{2}, \mathbb{Z}_{3}$.

Elliptic surfaces of this sort are called isotrivial. They explain the mechanism of creation of singular fibers of finite monodromy in arbitrary elliptic surfaces, as well as the occurrence of such fiber types in dual pairs $T, T^{*}$ with the same monodromy $\left(T=T^{*}\right.$ if $\left.\Gamma=\mathbb{Z}_{2} \subset \mathbb{R}\right)$. We already emphasize at this point that removing a type $T$ fiber leads to semi-flat metrics modelled on $(\mathbb{C} \times E) / \Gamma$ at infinity.

(i) $\Gamma=\mathbb{Z}_{2}$. Fix a smooth elliptic curve $E: y^{2}=g(x)$, where $g$ is a cubic, and fix an affine coordinate $t$ on $\mathbb{P}^{1}$ such that the natural $\mathbb{Z}_{2}$-action on $\mathbb{P}^{1}$ becomes $t \mapsto-t$. Setting $(T, X, Y)=\left(t^{2}, x, t y\right)$ yields a rational map $\mathbb{P}_{t}^{1} \times E \rightarrow \mathbb{P}_{T}^{1} \times \mathbb{P}_{X Y}^{2}$, which factors through the globally defined $\mathbb{Z}_{2}$-action $(t, x, y) \mapsto(-t, x,-y)$ and induces a birational map from $\left(\mathbb{P}_{t}^{1} \times E\right) / \mathbb{Z}_{2}$ to the surface $S: Y^{2}=T g(X)$, which is in fact an isomorphism of elliptic fibrations away from $t=0, \infty$. Project $S$ onto $\mathbb{P}_{X Y}^{2}$ and blow up the base points of the pencil $Y^{2}=T g(X)$. Going back then already yields a resolution for $\left(\mathbb{P}^{1} \times E\right) / \mathbb{Z}_{2}$, without having to blow up in a singular fiber.

To better understand the structure of the singular fibers, we begin by observing that the base points are $P_{i}=\left(x_{i}, 0\right)=\left(x_{i}: 0: 1\right)$ for $i=1,2,3$, where $g\left(x_{i}\right)=0$, and $P_{0}=(0: 1: 0)$. At $P_{i}$, the curves in the pencil have a common tangent $L_{i}$ : $X=x_{i}$ of multiplicity 2 , whereas at $P_{0}$ they share the line at infinity $L_{0}$ as a flex tangent. We blow up $P_{i}$ twice, with exceptional curves $E_{i}^{1}, E_{i}^{2}$, and $P_{0}$ three times, with exceptional curves $E_{0}^{1}, E_{0}^{2}, E_{0}^{3}$. The resulting elliptic fibration then has four sections, given by the $(-1)$-curves $E_{i}^{2}$ and $E_{0}^{3}$, and two singular fibers. The one at $T=0$ consists of a "spine", $Y=0$, and four "ribs" $E_{i}^{1}, L_{0}$ that intersect the spine transversely in four different points. The singular fiber at $T=\infty$ looks exactly the same, with $E_{0}^{1}$ as a spine and $L_{i}, E_{0}^{2}$ as ribs. Both fibers are of Kodaira type $\mathrm{I}_{0}^{*}$. In 
the resolution picture, the spines are the proper transforms of the rational curves $E / \mathbb{Z}_{2} \subset\left(\mathbb{P}_{t}^{1} \times E\right) / \mathbb{Z}_{2}$ over $t=0, \infty$, while the ribs resolve the singularities.

It is clear what background metric one would have to take in order to construct a complete Calabi-Yau metric when removing either one of the singular fibers. There are flat metrics on $\mathbb{C}_{t} \times E, \mathbb{C}_{s} \times E\left(s=\frac{1}{t}\right)$ with associated 2-forms $\Omega_{0}=d t \wedge d w$, $\Omega_{\infty}=d s \wedge d w$, with $d w$ an invariant 1-form on $E$. The key fact is that both $\Omega_{0}$ and $\Omega_{\infty}$ are invariant under the $\mathbb{Z}_{2}$-action; hence they lift to the rational elliptic surface with pole divisor the singular fiber at $T=\infty, 0$, respectively. One can thus use the flat orbifold $(\mathbb{C} \times E) / \mathbb{Z}_{2}$ as a background; cf. Remark 1.6(ii) and [5, 17, 24, 49.

(ii) $\Gamma=\mathbb{Z}_{3}$. Take $E: y^{2}+y=x^{3}$ and $(x, y) \mapsto\left(\zeta_{3} x, y\right)$ as a generator for $\Gamma$. Fix an affine coordinate $t$ on $\mathbb{P}^{1}$ such that the natural $\mathbb{Z}_{3}$-action on $\mathbb{P}^{1}$ becomes $t \mapsto \zeta_{3}^{2} t$. Then the rational map $\mathbb{P}_{t}^{1} \times E \rightarrow \mathbb{P}_{T}^{1} \times \mathbb{P}_{X Y}^{2}$ given by $(T, X, Y)=\left(t^{3}, t x, y\right)$ factors through the diagonal $\Gamma$-action and thus induces a birational map from $\left(\mathbb{P}_{t}^{1} \times E\right) / \Gamma$ to the surface $T\left(Y^{2}+Y\right)=X^{3}$ in $\mathbb{P}_{T}^{1} \times \mathbb{P}_{X Y}^{2}$. The pencil of cubics obtained from this by projection has three base points of multiplicity $3: P_{1}=(0,0)=(0: 0: 1)$, $P_{2}=(0,-1)=(0:-1: 1)$, and $P_{3}=(0: 1: 0)$, with corresponding flex tangents $L_{1}: Y=0, L_{2}: Y=-1$, and $L_{3}$, the line at infinity. Blowing up therefore produces three exceptional divisors $E_{i}^{1}, E_{i}^{2}, E_{i}^{3}$ at every $P_{i}$, where the $E_{i}^{3}$ become sections. The resulting singular fiber over $T=0$ has a spine-rib structure again, with $X=0$ as spine and three two-component ribs $E_{i}^{1} \cup E_{i}^{2}$ (Kodaira type IV*). The fiber over $T=\infty$ consists of $L_{1}, L_{2}, L_{3}$, intersecting in $(1: 0: 0) \in L_{3}$ (Kodaira type IV). To regard this as a resolution of singularities for $\left(\mathbb{P}^{1} \times E\right) / \Gamma$, we need to blow up the intersection of $L_{1}, L_{2}, L_{3}$ over $T=\infty$; the new exceptional divisor then represents the strict transform of the rational curve $E / \Gamma \subset\left(\mathbb{P}^{1} \times E\right) / \Gamma$ over $t=\infty$.

As for the semi-flat background metrics on $X$ with one singular fiber removed, there is a new phenomenon compared to the $\mathbb{Z}_{2}$ case. Among the rational 2 -forms $\Omega_{0}=d t \wedge d w, \Omega_{\infty}=d s \wedge d w\left(s=\frac{1}{t}\right)$ on $\mathbb{P}_{t}^{1} \times E$ associated with the standard flat metrics on $\mathbb{C}_{t} \times E, \mathbb{C}_{s} \times E$, only $\Omega_{0}$ is invariant under the $\mathbb{Z}_{3}$-action we are looking at, which is generated by $(t, w) \mapsto\left(\zeta_{3}^{2} t, \zeta_{3} w\right)$ or $(s, w) \mapsto\left(\zeta_{3} s, \zeta_{3} w\right)$ (cf. the three two-component ribs $E_{i}^{1} \cup E_{i}^{2}$ in the singular fiber over $T=0$, which correspond to crepant resolutions of the three $A_{2}$-singularities of $\left(\mathbb{P}_{t}^{1} \times E\right) / \Gamma$ over $\left.t=0\right)$. Thus, when removing the type IV fiber over $T=\infty$, then obviously the right metric $\omega_{0}$ to use as a background is the flat orbifold metric on $\left(\mathbb{C}_{t} \times E\right) / \Gamma$, which is a flat $T^{2}$ submersion over a flat 2 -cone of cone angle $1 / 3$. On the other hand, when removing the type IV* fiber over $T=0$, we would need to find $\omega_{\infty}$ with $\omega_{\infty}^{2}=\Omega \wedge \bar{\Omega}$, where $\Omega=\frac{1}{T} \Omega_{0}$ is the unique rational 2 -form on the rational elliptic surface with a simple pole along that fiber. Thus, it seems reasonable to take $\omega_{0}$ and conformally change the flat cone metric $|T|^{-\frac{4}{3}}|d T|^{2}$ on the base (which is complete at $T=\infty$ ) by $|T|^{-2}$, which results in a flat cone metric (complete at $T=0$ ) of cone angle $2 / 3$. It follows from Sections 3.2-3.3 that this is indeed what the appropriate $\omega_{\text {sf }}$ looks like.

We now turn to the structure of singular fibers in general. Kodaira's main result in 29 says the following: Let $f: U \rightarrow \Delta$ be an elliptic fibration over the unit disk, with a section $\sigma$, such that all fibers except possibly $D=f^{-1}(0)$ are smooth and $D$ does not contain any $(-1)$-curves. Then the pair $(f, \sigma)$ is isomorphic to a canonical form $(\bar{f}, \bar{\sigma})$ whose total space, $\bar{U}$, is birational to the quotient of the total space of an explicit elliptic fibration by a finite group related to the monodromy of $f$.

To explain the notion of monodromy, we begin by noting that $\left.U\right|_{\Delta^{*}} \cong L / \Lambda$, due to the existence of $\sigma$, where $L \rightarrow \Delta^{*}$ is a holomorphic line bundle and $\Lambda \subset L$ is a 
lattice bundle. Now $H^{1}\left(\Delta^{*}, \mathcal{O}^{*}\right)=0$, so $L \cong \mathcal{O}$ and $\left.U\right|_{\Delta^{*}} \cong\left(\Delta^{*} \times \mathbb{C}\right) /\left(\mathbb{Z} \tau_{1}+\mathbb{Z} \tau_{2}\right)$ for multivalued holomorphic functions $\tau_{1}, \tau_{2}$ on $\Delta^{*}$. The pair $T=\left(\tau_{1}, \tau_{2}\right) \in \mathbb{C}^{1 \times 2}$, assumed to be positively oriented, transforms as $T \mapsto T A$ with $A=\left(\begin{array}{ll}a & b \\ c & d\end{array}\right) \in \operatorname{SL}(2, \mathbb{Z})$ when going around the puncture once in the counterclockwise sense. The conjugacy class of $A$ in $\mathrm{SL}(2, \mathbb{Z})$ is then referred to as the monodromy of $f$.

The central fibers that can occur are classified by topological type as $\mathrm{I}_{b}(b \geq 0)$, II, III, IV, and corresponding "*-types," $\mathrm{I}_{b}^{*}, \ldots$, where $\mathrm{I}_{0}$ stands for a smooth central fiber. We refer to [3, 29, for a detailed description of the other types, including the multiplicities of $f$ along the individual components in the reducible case. Here, we only note that the topological type determines the monodromy, whose order as an element of $\mathrm{SL}(2, \mathbb{Z})$ is finite except in the $\mathrm{I}_{b}$ and $\mathrm{I}_{b}^{*}$ cases for $b \geq 1$. In Example 3.1. we have seen how the pairs $\left(\mathrm{I}_{0}^{*}, \mathrm{I}_{0}^{*}\right)$ and $\left(\mathrm{IV}, \mathrm{IV}^{*}\right)$ occur in rational elliptic surfaces birational to $\left(\mathbb{P}^{1} \times E\right) / \Gamma$ with $\Gamma=\mathbb{Z}_{2}, \mathbb{Z}_{3}$; the pairs (II, II*) and (III, III*) can be created in a similar fashion with $\Gamma=\mathbb{Z}_{6}$ and $\mathbb{Z}_{4}$, and the monodromy around both fibers is $\Gamma$ in each case. The $T^{2}$-bundles over $S^{1}$ obtained by restricting $U$ to a loop around 0 are orientable. They are flat (Bieberbach) manifolds if the monodromy is trivial or finite, nilmanifolds for $\mathrm{I}_{b}(b \geq 1)$, and infranil for $\mathrm{I}_{b}^{*}(b \geq 1)$.

If $X$ is rational elliptic, then the Euler numbers of the singular fibers must add up to $\chi(X)=12$. Persson [45. has determined all possible configurations of singular fibers; there are 279 in total, the generic one being $12 \times \mathrm{I}_{1}$. All finite monodromy types can occur, as well as $\mathrm{I}_{b}$ for $b \in\{1, \ldots, 9\}$ and $\mathrm{I}_{b}^{*}$ for $b \in\{1, \ldots, 4\}$.

We need to understand how Kodaira's canonical form $(\bar{f}, \bar{\sigma})$ for a given fibration $(f, \sigma)$ with a section is constructed. Write $\left.U\right|_{\Delta^{*}}=\left(\Delta^{*} \times \mathbb{C}\right) /\left(\mathbb{Z} \tau_{1}+\mathbb{Z} \tau_{2}\right)$ as before. Then $\tau:=\tau_{2} / \tau_{1}: \Delta^{*} \rightarrow \mathfrak{H}$ is multivalued holomorphic, taking values in the upper half-plane, and transforms as $\tau \mapsto(d \tau+b) /(c \tau+a)$ under the image $[A] \in \operatorname{PSL}(2, \mathbb{Z})$ of the monodromy when going around the puncture in the counterclockwise sense. Let $j: \mathfrak{H} \rightarrow \mathbb{C}$ denote the classical elliptic modular function, normalized such that $j(i)=1$ and $j\left(\zeta_{3}\right)=0$. Recall that $j$ is $\operatorname{PSL}(2, \mathbb{Z})$-invariant and unramified except for branch points of order 2,3 along the orbits of $i, \zeta_{3}$. It turns out that $\mathcal{J}:=j \circ \tau$ (Kodaira's functional invariant) is a single-valued meromorphic function on $\Delta^{*}$.

- If $\mathcal{J}(0) \in \mathbb{C} \backslash\{0,1\}$, then $\tau$ is single-valued and extends as a regular function on $\Delta$ with $\tau(0)$ not in the $\operatorname{PSL}(2, \mathbb{Z})$-orbits of $i, \zeta_{3}$. Thus, in particular, the stabilizer of $\tau(0)$ in $\operatorname{PSL}(2, \mathbb{Z})$ is trivial, so $A= \pm 1$. If $A=1$, then $\tau_{1}, \tau_{2}$ extend as single-valued functions on $\Delta$, and $U \cong(\Delta \times \mathbb{C}) /\left(\mathbb{Z} \tau_{1}+\mathbb{Z} \tau_{2}\right)$ with $D$ smooth $\left(\mathrm{I}_{0}\right)$. If $A=-1$, we first make a base change $z=u^{2}$ over $\Delta_{z}^{*}$. The lifted fibration $U^{\prime} \rightarrow \Delta_{u}^{*}$ extends to $\Delta_{u}$ with a smooth central fiber, $D^{\prime}$. The free $\mathbb{Z}_{2}$-action on $U^{\prime}$ over $\Delta_{u}^{*}$ also extends, but with four fixed points on $D^{\prime}$. The quotient is then an elliptic fibration over $\Delta_{z}$, isomorphic to $U$ over $\Delta_{z}^{*}$, but with four surface singularities of type $A_{1}$ over $z=0$. Resolving these singularities gives the desired normal form $\bar{U} \rightarrow \Delta_{z}$ with a type $\mathrm{I}_{0}^{*}$ central fiber. This is in complete analogy with Example 3.1(i).

- If $\mathcal{J}(0)=0$, then $\tau$ is possibly multivalued, but still regular at 0 , with $\tau(0)$ in the orbit of $\zeta_{3}$. [A] fixes $\tau(0)$, which in total yields six possibilities for $A \in \operatorname{SL}(2, \mathbb{Z})$ up to conjugation, including $A= \pm 1$. Each case can be treated as before: Make a base change $z=u^{\text {ord } A}$ to obtain an elliptic fibration $U^{\prime} \rightarrow \Delta_{u}^{*}$ that extends to $\Delta_{u}$ with a smooth central fiber, $D^{\prime}$. The group $\langle A\rangle$ acts on $U^{\prime}$ with fixed points on $D^{\prime}$, and the desired normal form $\bar{U}$ is obtained by minimally resolving the singularities of the quotient and blowing down (-1)-curves in the central fiber if necessary. The 
case $\mathcal{J}(0)=1$ is the same. Other than $\mathrm{I}_{0}, \mathrm{I}_{0}^{*}$ for $A= \pm 1$, the fiber types that can occur are II, III, IV, and their corresponding *-types, as in Example 3.1(ii).

- If $\mathcal{J}$ has a pole of order $b \geq 1$, then $A$ is conjugate to $\pm A_{b}, A_{b}:=\left(\begin{array}{ll}1 & b \\ 0 & 1\end{array}\right)$. Also, it is possible to make $\tau_{1} \equiv 1$ and $\tau_{2}=\tau=b(\log z) /(2 \pi i)$. If $b=1$ and $A=A_{1}$, one can fill in the surface $\left.U\right|_{\Delta^{*}} \cong\left(\Delta^{*} \times \mathbb{C}\right) /(\mathbb{Z}+\mathbb{Z} \tau)$ by using $\wp$-functions to imbed it into $\Delta \times \mathbb{P}^{2}$ and taking the closure. This yields the canonical form $\bar{U}$, with central fiber a node $\left(\mathrm{I}_{1}\right)$. The case $A=A_{b}(b>1)$, with central fiber a cycle of $b$ rational curves $\left(\mathrm{I}_{b}\right)$, reduces to $b=1$ by a fiberwise $b$-fold cover. $A=-A_{b}$ implies $A^{2}=A_{2 b}$, and $\bar{U}$ is obtained by setting $z=u^{2}$, which produces an $\mathrm{I}_{2 b}$ fibration over $\Delta_{u}$, then dividing out by $\mathbb{Z}_{2}$ and resolving the four resulting $A_{1}$-singularities $\left(\mathrm{I}_{b}^{*}\right)$.

3.2. Ricci-flat metrics on complex torus bundles. Let $X$ and $S$ be complex manifolds and let $f: X \rightarrow S$ be a holomorphic submersion such that all fibers $X_{s}$ $=f^{-1}(s)$ are complex $m$-tori. Suppose that $f$ admits a holomorphic section $\sigma$ and a constant polarization $\omega$, meaning that $\omega$ is a real 2-form on $X$ that restricts to a Kähler form on every fiber $X_{s}$, and that there exist $c_{i} \in \mathbb{R}$ and $\xi_{i}(s) \in H^{2}\left(X_{s}, \mathbb{Z}\right)$ such that $\left[\left.\omega\right|_{X_{s}}\right]=\sum c_{i} \xi_{i}(s)$ for each $s \in S$. For $\varepsilon>0$ and $s \in S$, we let $g_{s, \varepsilon}$ denote the unique flat Kähler metric on $X_{s}$ with $\operatorname{Vol}\left(X_{s}, g_{s, \varepsilon}\right)=\varepsilon$ whose Kähler class is a scalar multiple of $\left[\left.\omega\right|_{X_{s}}\right]$. Restricting $g_{s, \varepsilon}$ to $T_{\sigma(s)} X_{s}$ then induces a Hermitian fiber metric $h_{\varepsilon}$ on the holomorphic vector bundle $E:=\sigma^{*} T_{X / S}$ over $S$.

- Hyper-Kähler setting. Let $\operatorname{dim} S=m$ and assume that $X$ admits a holomorphic symplectic form $\Upsilon$ such that all $X_{s}$ are complex Lagrangians. Then there exists a unique Riemannian metric $g_{S, \varepsilon}$ on $S$, compatible with the complex structure on $S$, such that the faithful pairing $E \otimes T^{1,0} S \rightarrow \mathbb{C}$ given by $\Upsilon$ is isometric with respect to $h_{\varepsilon}$ and the Hermitian metric induced by $g_{S, \varepsilon}$.

- Calabi-Yau setting. Let $\operatorname{dim} S=1$ and suppose that $X$ admits a holomorphic volume form $\Omega$. Then $\Omega$ defines a faithful pairing $\wedge^{m} E \otimes T^{1,0} S \rightarrow \mathbb{C}$. Using the metric on $\wedge^{m} E$ induced by $h_{\varepsilon}$, we obtain a Riemannian metric $g_{S, \varepsilon}$ as before.

Using $g_{S, \varepsilon}$ and the family of flat metrics $\left\{g_{s, \varepsilon}\right\}$ on the fibers of $f$, we now wish to construct a submersion metric on $X$, so we need to specify a horizontal distribution. Choose a fiber-preserving biholomorphism $X \cong E / \Lambda$ for some holomorphic lattice bundle $\Lambda \subset E$. Then $\Lambda$ induces a flat $\mathbb{R}$-linear connection on $E$, hence an integrable horizontal distribution $\mathcal{H}$ on $X$, and this is independent of how we made $X \cong E / \Lambda$ since any two such isomorphisms only differ by a holomorphic section $S \rightarrow \operatorname{Aut}(E)$. Then $g_{\mathrm{sf}, \varepsilon}(u, v):=g_{S, \varepsilon}\left(f_{*} u, f_{*} v\right)+g_{s, \varepsilon}(P u, P v)$ for $u, v \in T_{x} X, s=f(x)$, and $P:=$ projection along $\mathcal{H}_{x}$, defines a semi-flat Hermitian metric, $g_{\mathrm{sf}, \varepsilon}$, on $X$. We will see that $g_{\mathrm{sf}, \varepsilon}$ and $g_{S, \varepsilon}$ are in fact Kähler and that the volume form of $g_{\mathrm{sf}, \varepsilon}$ is a constant multiple of $\Upsilon^{m} \wedge \bar{\Upsilon}^{m}$ or $\Omega \wedge \bar{\Omega}$, so that $\operatorname{Hol}\left(X, g_{\mathrm{sf}, \varepsilon}\right) \subset \mathrm{Sp}(m)$ or $\mathrm{SU}(m+1)$.

Remark 3.2. (i) Existence of a section is necessary to construct $g_{\mathrm{sf}, \varepsilon}$, and $g_{\mathrm{sf}, \varepsilon}$ as a tensor field depends on the particular choice of a section $\sigma$. However, $\sigma$ also defines a complex Lie group structure on each fiber $X_{s}$ with unit $\sigma(s)$, so for every smooth section $\sigma^{\prime}$ there is an associated vertical translation map, $T(x)=x+\sigma^{\prime}(f(x))$, and $g_{\mathrm{sf}, \varepsilon}[\sigma]=T^{*} g_{\mathrm{sf}, \varepsilon}\left[\sigma^{\prime}\right]$ if $\sigma^{\prime}$ is holomorphic. Also, T preserves $g_{\mathrm{sf}, \varepsilon}$ if and only if $\sigma^{\prime}$ is tangent to $\mathcal{H}$, which then implies that $\sigma^{\prime}$ is holomorphic.

(ii) The Hermitian metric $h_{\varepsilon}$ on $E$ induces the Weil-Petersson fiber metric on the first Hodge bundle $\wedge^{m} E^{*}$ up to a scale [50, p. 618]. This suggests that $\operatorname{Ric}\left(g_{S, \varepsilon}\right)$ is equal to the pullback of an invariant Kähler metric on the Siegel upper half-plane $\mathfrak{H}_{m}=\left\{Z \in \mathbb{C}^{m \times m}: Z^{\text {tr }}=Z, \operatorname{Im} Z>0\right\}$. We will prove this in Lemma 3.3(ii). 
We now derive an explicit formula for $g_{\mathrm{sf}, \varepsilon}$ in local coordinates, in terms of $\Upsilon, \Omega$ and the periods of the tori $X_{s}$ with respect to $\omega$. One can deduce from this formula that $g_{\mathrm{sf}, \varepsilon}$ is Kähler and identify the Ricci tensor of the metric $g_{S, \varepsilon}$ on the base with a natural Weil-Petersson metric, as mentioned in Remark 3.2 (ii).

Fix isomorphisms $\left.\left.X\right|_{U} \cong E\right|_{U} / \Lambda \cong\left(U \times \mathbb{C}^{m}\right) / \Lambda$ over a domain $U \subset \mathbb{C}^{m}, \mathbb{C}$, with coordinates $z$ on $U$ and $w$ along the fibers. Fix an oriented basis $\left(\tau_{1}, \ldots, \tau_{2 m}\right)$ for $\Lambda$ at one point, and extend it as a tuple of multivalued functions $\tau_{i} \in \mathcal{O}\left(U, \mathbb{C}^{m}\right)$ that generate $\Lambda$ everywhere. Let $\left(\xi^{1}, \ldots, \xi^{2 m}\right)$ be $\mathbb{R}$-dual to $\left(\tau_{1}, \ldots, \tau_{2 m}\right)$. Our assumption on the existence of a constant polarization means that there exists $Q \in \mathbb{R}^{2 m \times 2 m}$, $Q+Q^{\operatorname{tr}}=0$, such that $\omega=\frac{1}{2} \sum Q_{i j} \xi^{i} \wedge \xi^{j}$ restricts to a flat Kähler metric on each fiber. The $\omega$-volume of the fundamental cells is then given by the Pfaffian of $Q$.

To see more explicitly what this means, define $T \in \mathcal{O}\left(U, \mathbb{C}^{m \times 2 m}\right)$ by

$$
\tau_{i}=\left(T_{1 i}, \ldots, T_{m i}\right), \quad i=1, \ldots, 2 m .
$$

Since $\omega$ is well-defined, the monodromy of $T$ around closed loops is constrained by the condition that if $T$ transforms into $T A$ with $A \in \mathrm{GL}(2 m, \mathbb{R})$, then $A^{\operatorname{tr}} Q A=Q$. To translate the positive $(1,1)$ condition on $\omega$, we begin by noting that there exists an $S \in \mathrm{GL}(2 m, \mathbb{R})$, unique up to right multiplication by a matrix in $\operatorname{Sp}(2 m, \mathbb{R})$, such that $S^{\operatorname{tr}} Q S=\left(\begin{array}{cc}0 & 1 \\ -1 & 0\end{array}\right)$. We then write $T S=R(1, Z)$, with multivalued holomorphic maps $R: U \rightarrow \operatorname{GL}(m, \mathbb{C})$ and $Z: U \rightarrow \mathbb{C}^{m \times m}$. By Griffiths-Harris [18, Chapter 2.6], $\omega$ being positive $(1,1)$ is equivalent to $Z$ mapping into the Siegel upper half-plane; cf. Remark 3.2(ii). If this is the case, then, by the same calculations,

$$
\omega=i H_{j k} d w^{j} \wedge d \bar{w}^{k}, \quad H^{-1}:=2 \bar{R}(\operatorname{Im} Z) R^{\mathrm{tr}}=i \bar{T} Q^{-1} T^{\mathrm{tr}} .
$$

We will refer to $Z: U \rightarrow \mathfrak{H}_{m}$ as the period map, even though, strictly speaking, this is not a well-defined map. Changing the matrix $S$ or the local branch of $T$ used to construct $Z$ will conjugate $Z$ by a constant matrix in $\operatorname{Sp}(2 m, \mathbb{R})$.

It is easy to check that the flat connection given by $\Lambda$ has Christoffel symbols

$$
\Gamma_{i}(z, w)=\frac{\partial T}{\partial z^{i}}\left(\begin{array}{c}
T \\
\bar{T}
\end{array}\right)^{-1}\left(\begin{array}{c}
w \\
\bar{w}
\end{array}\right) \in \mathbb{C}^{m}, \quad i=1, \ldots, \operatorname{dim}_{\mathbb{C}} U
$$

i.e. that the vectors $\left(e_{i}, \Gamma_{i}(z, w)\right)$ span the horizontal space at $(z, w) \in U \times \mathbb{C}^{m}$.

Lemma 3.3 collects the resulting formulas for $g_{\mathrm{sf}, \varepsilon}$ and calculates the Ricci form of the metric on the base. In the hyper-Kähler setting, (ia), assuming $\Upsilon$ to have the particular form specified there, is a nontrivial condition, which can however always be satisfied by adapting the coordinates to $\Upsilon$. On the other hand, in (ib), the local expression for $\Omega$ will have the required form for every bundle chart $(z, w)$.

Lemma 3.3. For $H$ as in (3.1) and $\varepsilon>0$, put $H(\varepsilon):=(\varepsilon / \sqrt{\operatorname{det} Q})^{\frac{1}{m}} H$.

(ia) If $\Upsilon=g\left(d z^{1} \wedge d w^{1}+\cdots+d z^{m} \wedge d w^{m}\right)$ with $g: U \rightarrow \mathbb{C}$ holomorphic, then the Kähler form of the Hermitian metric $g_{\mathrm{sf}, \varepsilon}$ is given by

$$
\omega_{\mathrm{sf}, \varepsilon}=i|g|^{2} H(\varepsilon)_{j k}^{-1} d z^{j} \wedge d \bar{z}^{k}+i H(\varepsilon)_{j k}\left(d w^{j}-\Gamma_{p}^{j} d z^{p}\right) \wedge\left(d \bar{w}^{k}-\bar{\Gamma}_{q}^{k} d \bar{z}^{q}\right) .
$$

This is a closed form with top power $\left(\begin{array}{c}2 m \\ m\end{array}\right) \Upsilon^{m} \wedge \bar{\Upsilon}^{m}$, so $g_{\mathrm{sf}, \varepsilon}$ is hyper-Kähler.

(ib) If $\Omega=g d z \wedge d w^{1} \wedge \cdots \wedge d w^{m}$ with $g: U \rightarrow \mathbb{C}$ holomorphic, then the Kähler form of the Hermitian metric $g_{\mathrm{sf}, \varepsilon}$ is given by

$$
\omega_{\mathrm{sf}, \varepsilon}=i|g|^{2} \operatorname{det}(H(\varepsilon))^{-1} d z \wedge d \bar{z}+i H(\varepsilon)_{j k}\left(d w^{j}-\Gamma^{j} d z\right) \wedge\left(d \bar{w}^{k}-\bar{\Gamma}^{k} d \bar{z}\right) .
$$

This is a closed form with top power $(m+1) ! i^{(m+1)^{2}} \Omega \wedge \bar{\Omega}$, so $g_{\mathrm{sf}, \varepsilon}$ is Calabi-Yau. 
(ii) The metrics $g_{S, \varepsilon}$ on the base are Kähler as well. Their Ricci forms

$$
\rho\left(\omega_{S, \varepsilon}\right)=-i \partial \bar{\partial} \log \operatorname{det}(\operatorname{Im} Z)=\frac{i}{4}\left((\operatorname{Im} Z)^{a b} d Z_{b c} \wedge(\operatorname{Im} Z)^{c d} d \bar{Z}_{d a}\right),
$$

the pullback of an invariant Kähler metric on $\mathfrak{H}_{m}$ under the period map $Z$.

Proof. By calculation. Part (ii) follows quickly from $H^{-1}=2 \bar{R}(\operatorname{Im} Z) R^{\operatorname{tr}}$.

We will now make these formulas completely explicit in the elliptic surface case, which provides one of the main ingredients for Sections 3.3-3.4. The mathematical content of the following corollary is the same as in Gross-Wilson [20, Example 2.2], but the presentation there is somewhat inconvenient for us; cf. Remark 3.5 .

Corollary 3.4. Let $f: X \rightarrow S$ be an elliptic fibration over a Riemann surface with a holomorphic section $\sigma$ and no singular fibers. Let $\Omega$ be a holomorphic symplectic form on $X$. Let $\omega_{\mathrm{sf}, \varepsilon}$ be semi-flat hyper-Kähler constructed from $\sigma, \Omega / \sqrt{2}$, such that the fibers of $f$ have area $\varepsilon$ with respect to $\omega_{\mathrm{sf}, \varepsilon}$. Thus, in particular, $\omega_{\mathrm{sf}, \varepsilon}^{2}=\Omega \wedge \bar{\Omega}$. Let $U$ be a domain in $S$, let $z$ be a holomorphic coordinate on $U$, identify $U$ with its $z$-image, and fix an isomorphism of elliptic fibrations $\left.X\right|_{U} \cong\left(U \times \mathbb{C}_{w}\right) /\left(\mathbb{Z} \tau_{1}+\mathbb{Z} \tau_{2}\right)$ with multivalued functions $\tau_{1}, \tau_{2}$ such that $\left(\tau_{1}, \tau_{2}\right)$ is positively oriented and $\sigma$ maps to the zero section. Then $\Omega=g d z \wedge d w$ with $g: U \rightarrow \mathbb{C}$ holomorphic, and

$$
\begin{aligned}
& \omega_{\mathrm{sf}, \varepsilon}=i|g|^{2} \frac{\operatorname{Im}\left(\bar{\tau}_{1} \tau_{2}\right)}{\varepsilon} d z \wedge d \bar{z}+\frac{i}{2} \frac{\varepsilon}{\operatorname{Im}\left(\bar{\tau}_{1} \tau_{2}\right)}(d w-\Gamma d z) \wedge(d \bar{w}-\bar{\Gamma} d \bar{z}), \\
& \Gamma(z, w)=\frac{1}{\operatorname{Im}\left(\bar{\tau}_{1} \tau_{2}\right)}\left(\operatorname{Im}\left(\bar{\tau}_{1} w\right) \frac{d \tau_{2}}{d z}-\operatorname{Im}\left(\bar{\tau}_{2} w\right) \frac{d \tau_{1}}{d z}\right) .
\end{aligned}
$$

On $U$, the Ricci form of the induced metric $\omega_{S, \varepsilon}$ on the base is given by

$$
\rho\left(\omega_{S, \varepsilon}\right)=-i \partial \bar{\partial} \log \operatorname{Im}(\tau)=\frac{i}{4} \frac{d \tau \wedge d \bar{\tau}}{\operatorname{Im}(\tau)^{2}}
$$

the pullback of the hyperbolic metric of Gauß curvature -2 on the upper half-plane $\mathfrak{H}=\{\operatorname{Im}(\tau)>0\}$ under the period map $\tau=\frac{\tau_{2}}{\tau_{1}}: U \rightarrow \mathfrak{H}$.

Remark 3.5. We have tried to emphasize the point of view that $\omega_{\mathrm{sf}, \varepsilon}$ is an intrinsic object associated with holomorphic data $(X, f, \sigma, \Omega)$. Given any local bundle chart $(z, w)$, for instance the one that we will construct based on Kodaira's work [29] in Section 3.3, we then obtain a coordinate expression, (3.2), for $\omega_{\mathrm{sf}, \varepsilon}$.

The point of view taken in [20, Example 2.2] is that $\Omega$ induces an isomorphism $\Psi: E \rightarrow\left(T^{1,0} S\right)^{*}$ with $\Psi^{*} \Omega_{\text {can }}=\Omega$ for the standard symplectic form $\Omega_{\text {can }}$ on the cotangent bundle. Given any chart $z$ for $S$, we then have an associated chart $(z, w)$ for $\left(T^{1,0} S\right)^{*}$, and $\omega_{\mathrm{sf}, \varepsilon}$ is defined by a local expression in terms of this chart.

Semi-flat metrics play a role in a number of recent works $[14,17,20,25,40,50$. It is interesting to take a brief look at the connections between some of these.

- Collapsing Ricci-flat metrics on elliptic $K 3$ [20. Let $f: X \rightarrow \mathbb{P}^{1}$ be an elliptic fibration on a $K 3$ surface with a section and 24 type $\mathrm{I}_{1}$ singular fibers, and let $\Omega$ be a holomorphic symplectic form on $X$. Then the complement of the singular fibers carries semi-flat metrics $\omega_{\mathrm{sf}, \varepsilon}$ associated to $\Omega$ and a chosen section of $f$. Since $\Omega$ is regular, these metrics are incomplete at the singular fibers. They were used in 20 ] to construct a one-parameter family $\omega_{\varepsilon}$ of collapsing Ricci-flat metrics on $X$ which are increasingly well approximated by $\omega_{\mathrm{sf}, \varepsilon}$ as $\varepsilon \rightarrow 0$. Since $\mathrm{I}_{1}=\left(\begin{array}{ll}1 & 1 \\ 0 & 1\end{array}\right)$, the locally 
constant sheaf of isometric $T^{2}$-actions in Remark 3.2(i) has a section over $\Delta^{*}$. This global $S^{1}$-symmetry is crucial for modelling $\omega_{\varepsilon}$ at the singular fibers.

- Kähler-Ricci flow on properly elliptic surfaces [50]. Take $f: X \rightarrow \mathbb{P}^{1}$ to be any elliptic fibration on $K 3$, $[\omega]$ any Kähler class on $X$, and $\left[\beta_{0}\right]$ any Kähler class on $\mathbb{P}^{1}$. The methods of [50] show that the unique Ricci-flat metric in $[\omega]+t\left(f^{*}\left[\beta_{0}\right]-[\omega]\right)$ converges to $f^{*} \beta$ as $t \rightarrow 1$, away from the singular fibers, where

$$
\beta=\frac{\Omega \wedge \bar{\Omega}}{f^{*} \beta_{0} \wedge \omega_{\mathrm{SF}}} \beta_{0},
$$

and $\omega_{\mathrm{SF}}$ is any Kähler metric that restricts to a flat metric on every smooth fiber. It then follows from (3.2) that $\beta=\omega_{S, \varepsilon}$, with $S$ the set of regular values of $f$. This description will allow us to write down explicit expressions for $\beta$ in Section 3.3.

- Affine flat structures [14, 40. Given an elliptic fibration without singular fibers over some base $S$, equipped with a holomorphic symplectic form and a section, we get a flat connection on $\left(T^{1,0} S\right)^{*}$. By [40, the difference between this and the LeviCivita connection of $\beta:=\omega_{S, \varepsilon}$ can be viewed as a holomorphic cubic differential $U$ on $S$, and $\rho(\beta)=|U|^{2} \beta$ (Titeica's equation), where $|\ldots|^{2}$ denotes norm squared with respect to $\beta$. An observation in [40] states that this equation formally implies that $\rho(\rho(\beta))=-2 \rho(\beta)$ if $U \neq 0$. The cubic differential on $\mathbb{P}^{1}$ associated with a generic $K 3$ elliptic fibration has 24 poles, and these are cusps of the Weil-Petersson metric $\rho(\beta)$. However, due to branching of the period map $\tau: \mathbb{P}^{1} \rightarrow \mathfrak{H} / \operatorname{PSL}(2, \mathbb{Z})=\mathbb{C}$, the Weil-Petersson metric also has nonorbifold conical singularities $\left|z^{m} d z\right|^{2}, m>0$, at a finite number of smooth fibers, and $\sum m=18$ from Riemann-Hurwitz applied to $\tau$. These branch points are precisely the zeros of the cubic differential.

3.3. Explicit hyper-Kähler metrics near a special fiber. Let $f: U \rightarrow \Delta$ be an elliptic fibration over the disk with all fibers regular except possibly for $D=f^{-1}(0)$. Assume that $D$ does not contain $(-1)$-curves and is reduced or equivalently that $f$ admits a holomorphic section, or yet equivalently $\left.U\right|_{\Delta^{*}} \cong\left(\Delta^{*} \times \mathbb{C}\right) /\left(\mathbb{Z} \tau_{1}+\mathbb{Z} \tau_{2}\right)$ for some multivalued holomorphic functions $\tau_{1}, \tau_{2}: \Delta^{*} \rightarrow \mathbb{C}$. Let $\Omega$ be a meromorphic 2 -form on $U$ such that $\operatorname{div}(\Omega)$ is an integer multiple of $D$, and write $\Omega=g d z \wedge d w$, $g: \Delta^{*} \rightarrow \mathbb{C}$, where $z$ is the coordinate on $\Delta$ and $w$ the one along the fibers.

In this section, we either assume $\operatorname{div}(\Omega)=-D$, which is the interesting case on a rational elliptic surface, or $\operatorname{div}(\Omega)=0$, corresponding to the Gross-Wilson setting. Thanks to Kodaira's work 29], as summarized in Section 3.1, we can assume that $f: U \rightarrow \Delta$ belongs to a list of explicit model fibrations. For each member of this list, we then construct an explicit isomorphism $\Phi:\left.U\right|_{\Delta^{*}} \rightarrow\left(\Delta^{*} \times \mathbb{C}\right) /\left(\mathbb{Z} \tau_{1}+\mathbb{Z} \tau_{2}\right)$, meaning that both the map $\Phi$ and the functions $\tau_{1}, \tau_{2}$ will be explicit. This allows us to determine the multiplicity $N$ of $\Phi^{*}(d z \wedge d w)$ along $D$, so that $\Phi^{*}(d z \wedge d w)=$ $f^{N} h d x \wedge d y$ with $h(p) \neq 0$ in local coordinates $(x, y)$ on $U$ near each point $p \in D$. Thus, we either have $g(z)=z^{-N-1} k(z)$ or $g(z)=z^{-N} k(z), k(0) \neq 0$, according to whether $\operatorname{div}(\Omega)=-D$ or $\operatorname{div}(\Omega)=0$. Corollary 3.4 now gives formulas for $\omega_{\mathrm{sf}, \varepsilon}$ on $U \backslash D$ that are explicit up to higher-order terms from the Taylor series of $k$.

(1) Trivial monodromy. In this case, one can set things up such that $\tau_{1} \equiv 1$ and $\tau_{2}=\tau$, where $\tau: \Delta \rightarrow \mathfrak{H}$ is a regular function that satisfies $D \cong \mathbb{C} /(\mathbb{Z}+\mathbb{Z} \tau(0))$, but is not constrained otherwise; in particular, $N=0$. The $\operatorname{div}(\Omega)=0$ case is not interesting. If $\operatorname{div}(\Omega)=-D$, then $\Omega=z^{-1} k(z) d z \wedge d w$ with $k(0) \neq 0$; hence

$$
\omega_{\mathrm{sf}, \varepsilon}=i|k|^{2} \frac{\operatorname{Im}(\tau)}{\varepsilon} \frac{d z \wedge d \bar{z}}{|z|^{2}}+\frac{i}{2} \frac{\varepsilon}{\operatorname{Im}(\tau)}(d w-\Gamma d z) \wedge(d \bar{w}-\bar{\Gamma} d \bar{z}), \Gamma=\frac{\operatorname{Im}(w)}{\operatorname{Im}(\tau)} \frac{d \tau}{d z} .
$$


Note that $\mu:=|k(0)|(2 \operatorname{Im} \tau(0))^{\frac{1}{2}}$ has an intrinsic interpretation as $\mu^{2}=i \int_{D} R \wedge \bar{R}$ for the Poincaré residue $R$ of $\Omega$ along $D$. Then change coordinates on $\Delta \backslash[0,1)$ by setting $z=\exp \left(-u / \frac{\mu}{\sqrt{\varepsilon}}\right)$, with $u$ ranging in the strip $(0, \infty)+i\left(0,2 \pi \frac{\mu}{\sqrt{\varepsilon}}\right)$, so that

$$
\omega_{\mathrm{sf}, \varepsilon}=\frac{i}{2}\left(d u \wedge d \bar{u}+\frac{\varepsilon}{\operatorname{Im} \tau(0)} d w \wedge d \bar{w}\right)\left(1+O_{\varepsilon}\left(e^{-\frac{\sqrt{\varepsilon}}{\mu} \operatorname{Re} u}\right)\right) .
$$

The error estimate is understood to hold for all derivatives as well, with $w$ ranging in the fundamental domain spanned by $1, \tau(z(u))$ in $\{u\} \times \mathbb{C}$. Thus, $\omega_{\text {sf }, \varepsilon}$ decays to a flat cylinder $\mathbb{R}^{+} \times S^{1} \times D$, where $D$ carries its flat Kähler metric of area $\varepsilon$ and the circle has length $2 \pi \frac{\mu}{\sqrt{\varepsilon}}$, at the exponential rate $O_{\varepsilon}\left(\exp \left(-r / \frac{\mu}{\sqrt{\varepsilon}}\right)\right)$. If we replace $\Omega$ by $\alpha \Omega$, the circle gets scaled by $|\alpha|$ while the area of the torus stays fixed.

Recall from Section 3.1 that we write $T \mapsto T A$ for the monodromy around $z=0$ (in the counterclockwise sense) in an elliptic fibration $\left(\Delta^{*} \times \mathbb{C}\right) /\left(\mathbb{Z} \tau_{1}+\mathbb{Z} \tau_{2}\right)$, where $T=\left(\tau_{1}, \tau_{2}\right) \in \mathbb{C}^{1 \times 2}$ is positively oriented and $A=\left(\begin{array}{ll}a & b \\ c & d\end{array}\right) \in \mathrm{SL}(2, \mathbb{Z})$.

(2) Finite monodromy. The calculations for all types of singular fibers where $A$ is of finite order are fairly similar, so we only discuss the most complicated case in detail, namely, the Kodaira type II, for which the central fiber is a cusp. Much of what follows is from [29], but some extra work is needed to extract the data we are interested in, i.e. $\Phi, \tau_{1}, \tau_{2}, N$ in the notation introduced earlier.

Let $\mathcal{J}(0)=0$, mult $_{0} \mathcal{J}=m \in \mathbb{N}$. Classically, $j(\tau)=\left[\left(\tau-\zeta_{3}\right) /\left(\tau-\zeta_{3}^{2}\right)\right]^{3} h(j(\tau))$ with $h(0) \neq 0$ if $\tau$ is close to $\zeta_{3}$; cf. [28, Abschnitt I, Kapitel 2, §15]. Thus, changing coordinates on $\Delta$, we can make $\left(\tau-\zeta_{3}\right) /\left(\tau-\zeta_{3}^{2}\right)=z^{\frac{m}{3}}$, or in other words,

$$
\tau(z)=\zeta_{3} \frac{1-\zeta_{3} z^{\frac{m}{3}}}{1-z^{\frac{m}{3}}} .
$$

Going around $z=0$ counterclockwise once, the function $\tau$ transforms by

$$
\tau \mapsto A \tau=\frac{d \tau+b}{c \tau+a}, \quad A=\left(\begin{array}{ll}
a & b \\
c & d
\end{array}\right)= \begin{cases} \pm\left(\begin{array}{cc}
0 & 1 \\
-1 & 1
\end{array}\right) & \text { if } m \equiv 1(3), \\
\pm\left(\begin{array}{cc}
1 & -1 \\
1 & 0
\end{array}\right) & \text { if } m \equiv 2(3), \\
\pm 1 & \text { if } m \equiv 0(3) .\end{cases}
$$

If $\mathcal{J} \equiv 0$, or $m=\infty$ (the isotrivial case; cf. Example 3.1), any one of these six cases can occur, and the following discussion still applies with $z^{\infty}=u^{\infty}:=0$.

We focus on the first case with the plus sign, so $T \mapsto T A, A=\left(\begin{array}{cc}0 & 1 \\ -1 & 1\end{array}\right)$, ord $A=6$. The pullback $U^{\prime}$ of $\left.U\right|_{\Delta^{*}}$ under $z=u^{6}$ can be written as $\left(\Delta_{u}^{*} \times \mathbb{C}_{v}\right) /\left(\mathbb{Z}+\mathbb{Z} \tau\left(u^{6}\right)\right)$ and thus extends to the whole disk $\Delta_{u}$ with central fiber $\mathbb{C} /\left(\mathbb{Z}+\mathbb{Z} \zeta_{3}\right)$. The action of the monodromy on $U^{\prime}$ by deck transformations is generated by

$$
A(u, v)=\left(\zeta_{6} u, \frac{v}{c \tau\left(u^{6}\right)+a}\right)=\left(\zeta_{6} u, \zeta_{6} \frac{1-u^{2 m}}{1-\zeta_{3} u^{2 m}} v\right) ;
$$

indeed, $A(u, v)=\left(\zeta_{6} u, \lambda v\right)$ with $\lambda \in \mathbb{C}^{*}$ determined by $\left(1, \frac{d \tau+b}{c \tau+a}\right)=(\lambda, \lambda \tau)\left(\begin{array}{ll}a & b \\ c & d\end{array}\right)$. It is then easy to check that the fiberwise linear map

$$
\Phi: \Delta^{*} \times \mathbb{C} \rightarrow \Delta^{*} \times \mathbb{C},(u, v) \mapsto\left(u^{6},\left(1-u^{2 m}\right) u^{5} v\right),
$$


factors through the action generated by (3.5), inducing an isomorphism of elliptic fibrations $\Phi:\left.U\right|_{\Delta^{*}} \rightarrow\left(\Delta^{*} \times \mathbb{C}\right) /\left(\mathbb{Z} \tau_{1}+\mathbb{Z} \tau_{2}\right)$ with multivalued generators

$$
\begin{aligned}
& \tau_{1}(z)=\operatorname{pr}_{2} \Phi\left(z^{\frac{1}{6}}, 1\right)=\left(1-z^{\frac{m}{3}}\right) z^{\frac{5}{6}} \\
& \tau_{2}(z)=\operatorname{pr}_{2} \Phi\left(z^{\frac{1}{6}}, \tau(z)\right)=\zeta_{3}\left(1-\zeta_{3} z^{\frac{m}{3}}\right) z^{\frac{5}{6}} .
\end{aligned}
$$

What remains to be done before we can write down explicit formulas for the semiflat metrics is to find the multiplicity $N$ of $\Phi^{*}(d z \wedge d w)$ along the central fiber.

To do this, we recall that, by Kodaira's theorem, $U \rightarrow \Delta_{z}$ is obtained by dividing out $U^{\prime} \rightarrow \Delta_{u}$ by the monodromy action (3.5), which trivially recovers $U$ over $\Delta_{z}^{*}$, resolving the singularities that appear on the central fiber of the quotient, and then blowing down vertical ( -1$)$-curves if needed. Near its three orbits of fixed points, the action generated by (3.5) is conjugate to the $\mathbb{Z}_{6 / k}$-action on $\mathbb{C}^{2}$ generated by $(x, y) \mapsto\left(\zeta_{6}^{k} x, \zeta_{6}^{k} y\right)$ for $k=1,2,3$, respectively, where the germ of $x=0$ corresponds to the central fiber of $U^{\prime}$. To resolve the quotients, map

$$
(x, y) \mapsto\left(x^{\frac{6}{k}}, y^{\frac{6}{k}},(x: y)\right) \in \mathbb{C} \times \mathbb{C} \times \mathbb{P}^{1}, \quad k=1,2,3 .
$$

Globally, this produces exceptional curves $E_{1}, E_{2}, E_{3}$ and the strict transform $E$ of the central fiber of $U^{\prime} / \mathbb{Z}_{6}$. One checks that the multiplicities of $z \circ \Phi$ along these are $1,2,3,6$, whereas the multiplicities of $\Phi^{*}(d z \wedge d w)$ are $1,3,5,10$. It turns out that $E$ is a (-1)-curve. Blowing down $E$ turns $E_{3}$ into a (-1)-curve, blowing down $E_{3}$ turns $E_{2}$ into a (-1)-curve, and after blowing down $E_{2}$, the remaining curve $E_{1}$ becomes a rational cusp curve of self-intersection zero. This already shows that $N=1$. It is instructive to double check this result by considering $\Theta:=\left(y^{2}-x^{3}\right)^{N} d x \wedge d y$ on $\mathbb{C}^{2}$ and blowing up three times. The multiplicity of $\Theta$ is then $N$ along $E_{1}, 2 N+1$ along $E_{2}, 3 N+2$ along $E_{3}$, and $6 N+4$ along $E$.

We can now write down formulas for the semi-flat metrics. Since $N=1$, we have $\Omega=g d z \wedge d w$ with $g(z)=z^{-2} k(z)$ or $z^{-1} k(z)$, and $k(0) \neq 0$, if $\operatorname{div}(\Omega)=-D$ or 0 , respectively. In the first case, by (3.2), (3.3), and (3.6), (3.7),

$$
\begin{aligned}
& \omega_{\mathrm{sf}, \varepsilon}=i|k|^{2} \frac{\operatorname{Im}\left(\bar{\tau}_{1} \tau_{2}\right)}{\varepsilon} \frac{d z \wedge d \bar{z}}{|z|^{4}}+\frac{i}{2} \frac{\varepsilon}{\operatorname{Im}\left(\bar{\tau}_{1} \tau_{2}\right)}(d w-\Gamma d z) \wedge(d \bar{w}-\bar{\Gamma} d \bar{z}), \\
& \operatorname{Im}\left(\bar{\tau}_{1} \tau_{2}\right)=\frac{\sqrt{3}}{2}|z|^{\frac{5}{3}}\left(1-|z|^{\frac{2 m}{3}}\right), \quad \Gamma(z, w)=\frac{5}{6} \frac{w}{z}(1+\text { error }) .
\end{aligned}
$$

Here, error $=\frac{1}{w}(\operatorname{Re}(w) P+\operatorname{Im}(w) Q)$ with $P, Q=O\left(|z|^{\frac{m}{3}}\right)$. If $m=\infty$, so that the fibration becomes isotrivial, then error $=0$.

In order to better understand this metric, we change coordinates over $\Delta \backslash[0,1)$ by $z=\left(u / u_{0}\right)^{-6}, w=\left(u / u_{0}\right)^{-5} v$, with $u_{0}:=6 \sqrt[4]{3} \varepsilon^{-1 / 2}|k(0)|$. Here, $u$ ranges over the truncated sector $|u|>u_{0}, 0<\arg u<\frac{2 \pi}{6}$, and $v$ belongs to a fundamental cell in $\{u\} \times \mathbb{C}$ that converges to the one spanned by $1, \zeta_{3}$ as $|u| \rightarrow \infty$. Then

$$
\begin{aligned}
& \omega_{\mathrm{sf}, \varepsilon}=\frac{i}{2}\left(d u \wedge d \bar{u}+\frac{\varepsilon}{\operatorname{Im}\left(\zeta_{3}\right)} \eta \wedge \bar{\eta}\right)\left(1+O\left(|u|^{-4 m}\right)\right), \\
& \eta=d v-\Pi d u, \quad \Pi(u, v)=\frac{1}{u}(\hat{P} v+\hat{Q} \bar{v}), \quad \hat{P}, \hat{Q}=O\left(|u|^{-2 m}\right) .
\end{aligned}
$$

The $O$ notation here is understood to imply an improvement of $|u|^{-1}$ each time we differentiate with respect to either $u$ or $v$, and $\Pi=0$ in the isotrivial case. Hence, over the slit disk $\Delta \backslash[0,1), \omega_{\mathrm{sf}, \varepsilon}$ converges to a flat sector with opening angle $\frac{2 \pi}{6}$ in the plane, times a flat torus of area $\varepsilon$ homothetic to $\mathbb{C} /\left(\mathbb{Z}+\mathbb{Z} \zeta_{3}\right)$. To close the slit, we then simply twist by the monodromy, which is an isometry in this case. 
Notice that (3.10), (3.11) are not quite enough yet to establish that $\omega_{\mathrm{sf}, \varepsilon}$ is ALG in the sense of Definition 1.4(i). For this, we need to pull back by $(u, v) \mapsto\left(u, M_{u} v\right)$, where $M_{u} \in \mathrm{GL}(2, \mathbb{R}), M_{u}=1+O\left(|u|^{-2 m}\right)$, is defined by $w\left(M_{u}(1)\right)=\tau_{1}(z(u))$ and $w\left(M_{u}\left(\zeta_{3}\right)\right)=\tau_{2}(z(u))$. This verifies the $\operatorname{ALG}\left(2 m,\left[1 / 6, \varepsilon, \zeta_{3}\right]\right)$ property. Solving the Monge-Ampère equation changes the metric by $O\left(r^{-2-\delta}\right)$, by Proposition 2.9, and in actuality by no more than $O\left(r^{-8}\right)$; cf. Example 2.10.

To conclude, we briefly look at the Gross-Wilson setting, where $\operatorname{div}(\Omega)=0$. We only need to replace $|z|^{-4} d z \wedge d \bar{z}$ in (3.8) by $|z|^{-2} d z \wedge d \bar{z}$. The semi-flat metric is incomplete, and the metric on the base has a conical singularity with angle $\frac{5}{6}$.

All finite monodromies can be treated in this way, and lead to semi-flat metrics with similar properties. Table 1 lists $\tau_{1}, \tau_{2}, N$, and the tangent cones of the metrics on the base: incomplete $(\operatorname{div}(\Omega)=0)$, and complete $(\operatorname{div}(\Omega)=-D)$.

Remark 3.6. (i) Table 1 matches with Example 3.1 The complete semi-flat metric obtained by removing a fiber of type $\mathrm{I}_{0}^{*}$, II, III, or IV is asymptotic to a flat orbifold $(\mathbb{C} \times E) / \mathbb{Z}_{k}$, where $E$ is a suitable flat complex torus and the $\mathbb{Z}_{k}$-action conjugates into SU(2). Removing the dual fiber instead yields an asymptotic cone angle $1-\frac{1}{k}$. Example 3.1 also explains why the complete cone angle when removing one fiber is equal to the incomplete cone angle when removing its dual.

(ii) According to Table 1, the estimate (3.17) in Song-Tian [50] is incorrect, but the error is irrelevant for the rest of that paper. Indeed, if we define $B, s, F$ as in [50] and let $g$ denote the horizontal component of the incomplete semi-flat metric on $B$, then $F|d s|^{2}=e^{\psi} g$ with $\psi \in C^{\infty}(B)$, so that $F \sim|s|^{-\alpha}$ for some $\alpha>0$.

(3) Infinite monodromy. We will first derive explicit expressions for the semi-flat metrics associated with $\mathrm{I}_{b}$ and $\mathrm{I}_{b}^{*}$, and then discuss their geometry.

For any $\mathrm{I}_{1}$ degeneration of elliptic curves $f: U \rightarrow \Delta$, the restriction $\left.U\right|_{\Delta^{*}}$ must be abstractly isomorphic to $\left(\Delta^{*} \times \mathbb{C}\right) /\left(\mathbb{Z} \tau_{1}+\mathbb{Z} \tau_{2}\right)$, where $\tau_{1}=1$ and $\tau_{2}=\frac{1}{2 \pi i} \log z$. Kodaira also proved that the map

$$
\Psi(z, w):=\left(z,-\frac{1}{12}-\frac{1}{4 \pi^{2}} \wp_{z}(w), \frac{i}{8 \pi^{3}} \wp_{z}^{\prime}(w)\right) \in \Delta \times \mathbb{C}_{x y}^{2}
$$

induces an isomorphism from $\left(\Delta^{*} \times \mathbb{C}\right) /\left(\mathbb{Z} \tau_{1}+\mathbb{Z} \tau_{2}\right)$ onto the surface

$$
y^{2}=4 x^{3}+x^{2}-g_{2}(z) x-g_{3}(z)
$$

in $\Delta^{*} \times \mathbb{P}_{x y}^{2}$ with its natural elliptic fibration given by $\mathrm{pr}_{1}$, where $g_{2}, g_{3}$ are certain regular functions with $g_{2}(0)=g_{3}(0)=0$ and $\wp_{z}$ is the usual Weierstraß $\wp$-function associated with the lattice generated by $\tau_{1}(z), \tau_{2}(z)$. The closure of $\operatorname{im}(\Psi)$ is then a smooth elliptic surface $\bar{U}$ with central fiber the node $y^{2}=4 x^{3}+x^{2}$, representing Kodaira's canonical form for $\mathrm{I}_{1}$. In keeping with our earlier conventions, $\Phi:=\Psi^{-1}$ is the map of interest. To determine the multiplicity $N$ of $\Phi^{*}(d z \wedge d w)$ along $D$, it suffices to compute that $\Psi^{*} \Theta=2 \pi i d z \wedge d w$, where $\Theta:=d z \wedge(d x / y)$ obviously has multiplicity zero along $D$. Thus, $N=0$. By Kodaira again, the $\mathrm{I}_{b}$ case with $b>1$ reduces to $\mathrm{I}_{1}$ by a fiberwise $b$-fold unramified covering. Thus,

$$
\begin{aligned}
& \omega_{\mathrm{sf}, \varepsilon}=i|k|^{2} \frac{b|\log | z||}{2 \pi \varepsilon} \frac{d z \wedge d \bar{z}}{|z|^{2}}+\frac{i}{2} \frac{2 \pi \varepsilon}{b|\log | z||}(d w-\Gamma d z) \wedge(d \bar{w}-\bar{\Gamma} d \bar{z}), \\
& \Gamma(z, w)=\frac{1}{i} \frac{\operatorname{Im}(w)}{z|\log | z||}
\end{aligned}
$$




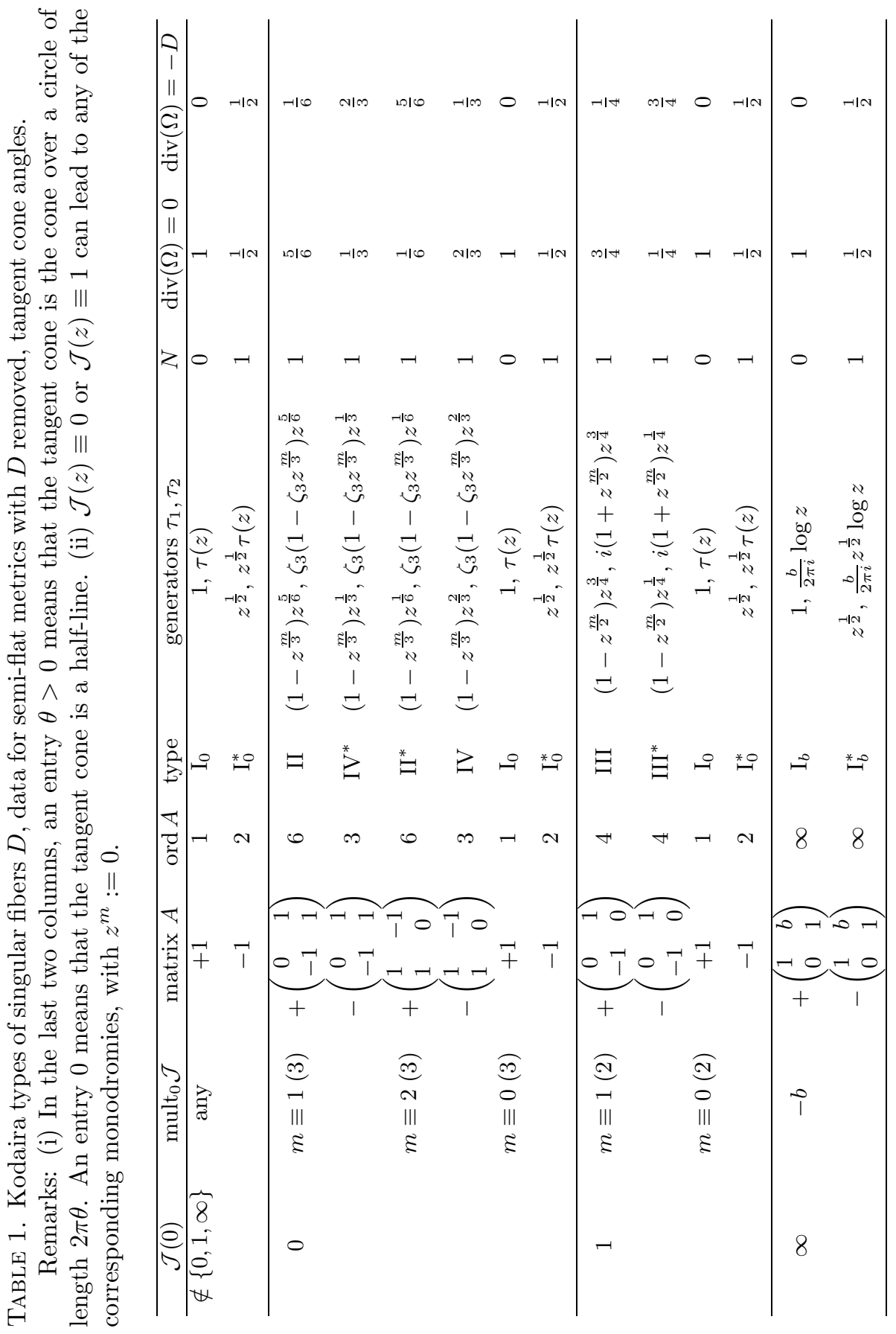


yields the complete $\mathrm{I}_{b}$ semi-flat metric with $\operatorname{div}(\Omega)=-D$. Replacing $|z|^{-2} d z \wedge d \bar{z}$ by $d z \wedge d \bar{z}$, we return to the incomplete setting that was originally studied in 20]; we only note here that the metric on the base satisfies $d^{2} K_{\mathrm{Gau}} \sim|\log d|^{-1}$ in that case, $d=\operatorname{dist}(0,-)$, and has tangent cone $\mathbb{R}^{2}$ at the origin; see also [46].

For a degeneration $f: U \rightarrow \Delta$ with $\mathrm{I}_{b}^{*}$ monodromy, the pullback of $\left.U\right|_{\Delta^{*}}$ under $z=u^{2}$ extends as an $\mathrm{I}_{2 b}$ fibration $U^{\prime} \rightarrow \Delta_{u}$, and $\mathbb{Z}_{2}$ acts naturally on $U^{\prime}$ such that the quotient has four ordinary double points on its central fiber. Kodaira's normal form $\bar{U}$ is obtained by resolving these singularities. Now, according to what we said about the $\mathrm{I}_{2 b}$ case, $\left.U^{\prime}\right|_{\Delta^{*}}=\left(\Delta^{*} \times \mathbb{C}_{v}\right) /\left(\mathbb{Z} \tau_{1}+\mathbb{Z} \tau_{2}\right)$ with $\tau_{1}=1$ and $\tau_{2}=\frac{b}{\pi i} \log u$. Moreover, since the volume form $d u \wedge d v$ on $U^{\prime}$ is invariant under the $\mathbb{Z}_{2}$-action, it pushes down to the quotient and lifts to a volume form on the (crepant) resolution $\bar{U}$. Thus, if $\Omega$ is a meromorphic 2-form on $\bar{U}$ as usual, then $\Omega=g d u \wedge d v$ upstairs on $U^{\prime}$ with $g(u)=u^{-2} k\left(u^{2}\right)$ or $g(u)=k\left(u^{2}\right)$ for a regular function $k$ with $k(0) \neq 0$, depending on whether $\operatorname{div}(\Omega)=-D$ or $\operatorname{div}(\Omega)=0$. In the first (i.e. complete) case, we then obtain the following expression for the associated semi-flat metric; we are working on the fundamental domain $\Delta^{+} \times \mathbb{C}$ for the $\mathbb{Z}_{2}$-action upstairs, where $\Delta^{+}$ denotes the right half-disk $\{u \in \Delta: \operatorname{Re} u>0\}$ :

$$
\begin{aligned}
& \omega_{\mathrm{sf}, \varepsilon}=i\left|k\left(u^{2}\right)\right|^{2} \frac{b|\log | u||}{\pi \varepsilon} \frac{d u \wedge d \bar{u}}{|u|^{4}}+\frac{i}{2} \frac{\pi \varepsilon}{b|\log | u||}(d v-\Gamma d u) \wedge(d \bar{v}-\bar{\Gamma} d \bar{u}) \\
& \Gamma(u, v)=\frac{1}{i} \frac{\operatorname{Im}(v)}{u|\log | u||}
\end{aligned}
$$

One can of course push this down to $\Delta_{z}^{*} \times \mathbb{C}_{w}$ again by mapping $(z, w)=\left(u^{2}, u v\right)$, so that $d z \wedge d w=2 u^{2} d u \wedge d v$ and hence $N=1$, which is the presentation we have given in Table 1 for the sake of analogy with the finite monodromy cases, especially $\mathrm{I}_{0}^{*}$; however, (3.14) and (3.15) are better suited for computations. We note that the metric for $\operatorname{div}(\Omega)=0$ is simply a $\mathbb{Z}_{2}$-quotient of the incomplete $\mathrm{I}_{b}$ metric.

We now discuss the asymptotic geometry of the complete $\mathrm{I}_{b}$ and $\mathrm{I}_{b}^{*}$ metrics from (3.12) and (3.14). The fibers now diverge in the moduli space of flat tori of constant area, i.e. the intrinsic length of one edge of any fundamental cell tends to $\infty$, while the length of the other edge, corresponding to the monodromy invariant period $\tau_{1}$, goes to zero. This effect is sublinear in the distance from any fixed base point, so in particular the asymptotic cones are $\mathbb{R}^{+}, \mathbb{R}^{2} / \mathbb{Z}_{2}$, as for the complete $\mathrm{I}_{0}, \mathrm{I}_{0}^{*}$ metrics, but it makes other features of the geometry somewhat tricky to understand.

We will determine $|\mathrm{Rm}|$ by calculating the squared norm of the curvature form $\Theta$ of the Chern connection. Here are the precise formulas we will use for this:

$$
\Theta=\partial\left(g^{-1} \bar{\partial} g\right), \quad\left|A d z^{j} \wedge d \bar{z}^{k}\right|^{2}=g^{j \bar{j}} g^{k \bar{k}} \operatorname{trace}\left(g^{-1} A^{\operatorname{tr}} g \bar{A}\right),
$$

for any matrix $A \in \mathbb{C}^{m \times m}$, viewed as an endomorphism of the tangent space. Also, due to the local isometric $T^{2}$-action, it suffices to evaluate $|\Theta|^{2}$ at $w=0$, where the forms $d z \wedge d \bar{z}, d z \wedge d \bar{w}, d w \wedge d \bar{w}$ are orthogonal with respect to $g_{\mathrm{sf}, \varepsilon}$.

- Geometry of the complete $\mathrm{I}_{b}$ metrics. For the sake of reference, note that

$$
\int \frac{1}{t}\left(\log \frac{1}{t}\right)^{\frac{1}{2}} d t=-\frac{2}{3}\left(\log \frac{1}{t}\right)^{\frac{3}{2}}, \int \frac{1}{t} \log \frac{1}{t} d t=-\frac{1}{2}\left(\log \frac{1}{t}\right)^{2} .
$$


We will abuse notation by writing $\Delta^{*}=\left\{0<|z| \leq \frac{1}{2}\right\}$ and $U=f^{-1}\left(\Delta^{*}\right)$. We then define $z_{x}:=f(x) \in \Delta^{*}$ for all $x \in U$, and

$$
g:=\frac{b}{2 \pi \varepsilon}|k(z)|^{2} \frac{|\log | z||}{|z|^{2}}|d z|^{2},
$$

so that $f:\left(U, g_{\mathrm{sf}, \varepsilon}\right) \rightarrow\left(\Delta^{*}, g\right)$ is a Riemannian submersion. Finally, we assume for convenience that $\left(U, g_{\mathrm{sf}, \varepsilon}\right)$ is imbedded into a complete Riemannian manifold $M$ as the only end of $M$; fixing a base point $x_{0} \in U$, we then let $r_{x}:=\operatorname{dist}_{M}\left(x_{0}, x\right)$.

For every $z \in \Delta^{*}$, the intrinsic diameter of the fiber $f^{-1}(z)$ and the $g$-length of the Euclidean circle of radius $|z|$ in $\Delta^{*}$ are both $\sim|\log | z||^{\frac{1}{2}}$. Thus, by (3.17),

$$
\left.\left|z_{x}\right| \ll 1 \Longrightarrow r_{x} \sim \operatorname{dist}_{g}\left(z_{x}, z_{x_{0}}\right) \sim|\log | z_{x}\right|^{\frac{3}{2}}
$$

Also, $\operatorname{Vol}\left(f^{-1}(B), g_{\mathrm{sf}, \varepsilon}\right)=\varepsilon \cdot \operatorname{Vol}(B, g)$ for every $B \subset \Delta^{*}$ because $f$ is a Riemannian submersion with fibers of area $\varepsilon$. Hence, using (3.17) again,

$$
s \gg 1 \Longrightarrow\left|B\left(x_{0}, s\right)\right| \sim s^{\frac{4}{3}} .
$$

Taken together, these properties already imply that $M$ satisfies $\operatorname{CYL}\left(\frac{1}{3}\right)$ according to Definition 2.5] as well as the diameter growth condition from Definition 2.8.

In order to also establish property $\operatorname{SOB}\left(\frac{4}{3}\right)$ from Definition 2.3 , we additionally need an appropriate lower bound on the volume of large balls with far-out centers. To prove this bound, it suffices to show that

$$
r_{x} \gg 1 \Longrightarrow B\left(x, \frac{1}{2} r_{x}\right) \supset\left\{y \in U:\left|z_{x}\right|<\left|z_{y}\right|<\left|z_{x}\right|^{1-\alpha}\right\}
$$

for some fixed $\alpha<1$. This in turn is easy to see, using again that the diameter of $f^{-1}(z)$ as well as the $g$-length of the circle of radius $|z|$ in $\Delta^{*}$ are $\sim|\log | z||^{\frac{1}{2}}$.

Next, we determine $|\mathrm{Rm}|$ from the norm of the Chern curvature: As $r_{x} \rightarrow \infty$,

$$
|\Theta(x)|^{2}=\frac{6 \pi^{2} \varepsilon^{2}}{b^{2}|k(0)|^{4}}|\log | z_{x} \|^{-6}(1+o(1)) \sim r_{x}^{-4} .
$$

This was calculated on a computer, proceeding from (3.16).

As for the injectivity radius, we have

$$
\operatorname{inj}(x) \sim|B(x, 1)| \sim r_{x}^{-\frac{1}{3}} .
$$

The point here is that the metric $g$ on the base has positive injectivity radius, while the fibers are almost totally geodesic flat tori of constant area whose shortest closed geodesic is given by the monodromy invariant generator $\tau_{1}$.

To make this rigorous, note that $\operatorname{inj}(x) \gtrsim|B(x, 1)|$ from [9, Theorem 4.3] because $|\mathrm{Rm}| \leq C$, and one easily finds a subset of $B(x, 1)$ with sufficient volume. On the other hand, if $\gamma:[0, t] \rightarrow U$ with $\gamma(0)=\gamma(t)=x$ and $|\dot{\gamma}|=1$ is a shortest closed geodesic for the flat metric on the fiber through $x$, then $|\ddot{\gamma}| \leq C$ because the second fundamental form of the fiber is bounded (by Gauß-Codazzi and because the fiber is holomorphic). This then implies that $\operatorname{inj}(x) \leq C t$; indeed, if not, we could use normal coordinates to view $\gamma$ as a loop in $\mathbb{R}^{4}$ such that $|\dot{\gamma}| \geq \frac{1}{C}$ and $|\ddot{\gamma}| \leq C$ with respect to $g_{\mathbb{R}^{4}}$, which implies a contradiction once $t$ is sufficiently small.

To conclude, we compute the asymptotic cone of $M$. Since the intrinsic diameter of the fiber through $x$ is merely $o\left(r_{x}\right)$, it suffices to show that the pointed rescalings $\left(\Delta^{*}, \lambda^{2} g, \frac{1}{2}\right)$ converge in the pointed Gromov-Hausdorff sense as $\lambda \rightarrow 0$. Define

$$
\Phi_{\lambda}:[0, \infty) \times S^{1} \ni(s, \theta) \mapsto \frac{1}{2} \exp \left(-\lambda^{-\frac{2}{3}} s^{\frac{2}{3}}\right) e^{i \theta} \in \Delta^{*} .
$$


It is easy to check that $\Phi_{\lambda}^{*}\left(\lambda^{2} g\right)$ converges to $C d s^{2}$ as smooth tensor fields, locally uniformly on $(0, \infty) \times S^{1}$. Thus, $M$ has a unique tangent cone, given by $\mathbb{R}^{+}$.

- Geometry of the complete $\mathrm{I}_{b}^{*}$ metrics. We begin by noting that

$$
\begin{aligned}
& \int_{s}^{s_{0}} \frac{1}{t^{2}}\left(\log \frac{1}{t}\right)^{\frac{1}{2}} d t=\frac{1}{s}|\log s|^{\frac{1}{2}}\left(1+O_{s_{0}}\left(|\log s|^{-1}\right)\right), \\
& \int \frac{1}{t^{3}} \log \frac{1}{t} d t=-\frac{1}{2 t^{2}} \log \frac{1}{t}+\frac{1}{4 t^{2}} .
\end{aligned}
$$

Using notation analogous to our discussion of the $\mathrm{I}_{b}$ metrics, (3.18) yields

$$
\left.\left|u_{x}\right| \ll 1 \Longrightarrow r_{x} \sim \operatorname{dist}_{g}\left(u_{x}, u_{x_{0}}\right) \sim \frac{1}{\left|u_{x}\right|}|\log | u_{x}\right|^{\frac{1}{2}}
$$

since the fiber over $u \in \Delta^{+}$has diameter $\sim|\log | u||^{1 / 2}$, and the length of the halfcircle of radius $|u|$ in $\Delta^{+}$is $\sim|u|^{-1}|\log | u||^{1 / 2}$. In particular, the fiber has diameter $\sim\left(\log r_{x}\right)^{1 / 2}$, whereas the $g$-length of the half-circle is $\sim r_{x}$, i.e. precisely linear in the distance from any fixed base point rather than sublinear. Notice that this still suffices for Proposition 2.9(ii), but $M$ is then certainly not $\operatorname{CYL}(\gamma)$ for any $\gamma$.

From the above and (3.19), we deduce that

$$
s \gg 1 \Longrightarrow\left|B\left(x_{0}, s\right)\right| \sim s^{2} .
$$

In fact, we can even prove that $M$ is $\mathrm{SOB}(2)$ by observing that we now have

$$
\left|B\left(x, \frac{1}{2} r_{x}\right)\right| \supset\left\{y \in U:\left|u_{x}\right|<\left|u_{y}\right|<(1+\alpha)\left|u_{x}\right|,\left|\arg \frac{u_{y}}{u_{x}}\right|<\alpha\right\},
$$

for all $x \in U$ with $r_{x} \gg 1$ and some fixed, small $\alpha>0$. The Chern curvature

$$
|\Theta(x)|^{2}=\left.\frac{\pi^{2} \varepsilon^{2}}{2 b^{2}|k(0)|^{4}}\left|u_{x}\right|^{4}|\log | u_{x}\right|^{-4}(1+o(1)) \sim r_{x}^{-4}\left(\log r_{x}\right)^{-2}
$$

as $r_{x} \rightarrow \infty$. For the same general reason as before,

$$
\operatorname{inj}(x) \sim|B(x, 1)| \sim\left(\log r_{x}\right)^{-\frac{1}{2}} .
$$

The asymptotic cone of $M$ is obtained as the pointed limit of $\left(\Delta^{+}, \lambda^{2} g, \frac{1}{2}\right)$ as $\lambda \rightarrow 0$. If we define $u_{\lambda} \in \mathbb{R}^{+}$by $u_{\lambda}^{2}|\log | u_{\lambda}||^{-1}=\lambda^{2}$ and then let

$$
\Phi_{\lambda}:\left\{|u| \geq u_{\lambda}, \operatorname{Re} u>0\right\} \ni u \mapsto \frac{u_{\lambda}}{2 u} \in \Delta^{+},
$$

$\Phi_{\lambda}^{*}\left(\lambda^{2} g\right)$ will converge to $C|d u|^{2}$ locally uniformly as smooth tensors on $\{\operatorname{Re} u>0\}$. Thus, $M$ has a unique asymptotic cone, given by $\mathbb{R}^{2} / \mathbb{Z}_{2}$.

3.4. Existence and asymptotics. Let $f: X \rightarrow \mathbb{P}^{1}$ be the rational elliptic surface obtained by blowing up the base points of a pencil of cubics, $p \in \mathbb{P}^{1}, D:=f^{-1}(p)$. Fix a disk $\Delta=\{|z|<1\} \subset \mathbb{P}^{1}$ with $z(p)=0$ such that all fibers of $f$ over $\Delta^{*}$ are smooth, and write $\left.X\right|_{\Delta^{*}}=\left(\Delta^{*} \times \mathbb{C}\right) /\left(\mathbb{Z} \tau_{1}+\mathbb{Z} \tau_{2}\right)$ with $\tau_{1}, \tau_{2}$ from Table 1 . Let $\Omega$ be a rational 2 -form with $\operatorname{div}(\Omega)=-D$. Fix any Kähler metric $\omega$ on $M:=X \backslash D$ such that $\int_{M} \omega^{2}<\infty$ and $\int_{C} \omega=0$ for all bad cycles $C$, e.g. the restriction to $M$ of a Kähler metric on $X$. Choose a holomorphic section $\sigma: \Delta^{*} \rightarrow X$.

For all $\alpha>0$, we then get an associated semi-flat metric $\omega_{\mathrm{sf}}(\alpha)$ on $\left.X\right|_{\Delta^{*}}$, which as a tensor field depends on our choice of $\sigma$, such that $\omega_{\text {sf }}(\alpha)^{2}=\alpha \Omega \wedge \bar{\Omega}$ and such that the fibers of $f$ have area $\varepsilon$ with respect to $\omega_{\mathrm{sf}}(\alpha)$, where $\varepsilon$ denotes the area of the fibers of $f$ with respect to $\omega$. We studied the geometry of $\omega_{\mathrm{sf}}(\alpha)$ in Section 3.3. 
Our goal is to glue $\omega$ and $\omega_{\text {sf }}(\alpha)$ so as to produce a complete Kähler metric $\omega_{0}$ on $M$ to which the existence and decay results from Section 2.2 can be applied.

Proposition 3.7. There exist a holomorphic section $\tilde{\sigma}$ over $\Delta^{*}$, concentric disks $\Delta^{\prime} \subset \Delta^{\prime \prime} \subset \Delta^{\prime \prime \prime} \subset \Delta, a(1,1)$-form $\beta \geq 0$ on $\mathbb{P}^{1}$ such that $\operatorname{supp}(\beta) \subset \Delta^{\prime \prime \prime} \backslash \Delta^{\prime}$, and a constant $\alpha_{0}>0$, such that for all $\alpha>\alpha_{0}$ there exist $u_{\alpha}^{\text {int }} \in C^{\infty}\left(f^{-1}\left(\mathbb{P}^{1} \backslash \Delta^{\prime}\right), \mathbb{R}\right)$ and $u_{\alpha}^{\mathrm{ext}} \in C^{\infty}\left(f^{-1}\left(\Delta^{\prime \prime} \backslash\{0\}\right), \mathbb{R}\right)$ with the same complex Hessian over $\Delta^{\prime \prime} \backslash \Delta^{\prime}$, and $t_{\alpha}>0$, such that the closed $(1,1)$-form $\omega_{0}(\alpha, t):=\omega+t f^{*} \beta+i \partial \bar{\partial} u_{\alpha}^{\text {int,ext }}$ is positive for $t>t_{\alpha}, \omega_{0}(\alpha, t)=\omega$ over $\mathbb{P}^{1} \backslash \Delta^{\prime \prime \prime}$, and $\omega_{0}(\alpha, t)=T^{*} \omega_{\mathrm{sf}}(\alpha)$ over $\Delta^{\prime} \backslash\{0\}$, with $T$ denoting vertical translation by $\tilde{\sigma}$ relative to $\sigma$ as in Remark $3.2(i)$. In addition, we have $\int_{M}\left(\omega_{0}(\alpha, t)^{2}-\alpha \Omega \wedge \bar{\Omega}\right)=0$ for precisely one $t>t_{\alpha}$.

The basic idea is that there exist two obstructions against gluing $\omega$ and $\omega_{\mathrm{sf}}(\alpha)$ as closed $(1,1)$-forms. These live in $H^{2}\left(\left.X\right|_{\Delta^{*}}, \mathbb{R}\right)$ and $H^{0,1}\left(\left.X\right|_{\Delta^{*}}\right)$. We have in fact already set things up in such a way that the topological obstruction vanishes; as we will see, $H_{2}\left(\left.X\right|_{\Delta^{*}}, \mathbb{Z}\right)$ is generated by the class of the fibers and by the bad cycles, $C$, for which $\left\langle\omega_{\mathrm{sf}}(\alpha), C\right\rangle=0$ by computation. The analytic obstruction is killed by translating $\omega_{\text {sf }}(\alpha)$ by a section $\tilde{\sigma}$, balancing the freedom we had in our choice of $\sigma$. So far, this is a straightforward extension of work in [20].

One issue not present in [20] is that $\omega$ and $\omega_{\text {sf }}(\alpha)$ are not close on their overlap. Thus, gluing them by cutting off a Kähler potential for their difference may create large negative $d z \wedge d \bar{z}$ components. We balance these by adding $t f^{*} \beta$ with a bump form $\beta \geq 0$ on $\mathbb{P}^{1}, t \gg 1$. Then the point is that, by doing everything carefully, the condition $\int\left(\omega_{0}(\alpha, t)^{2}-\alpha \Omega \wedge \bar{\Omega}\right)=0$ becomes linear in $\alpha$ and $t$, with positive slope. (Notice that $T^{*} \Omega=\Omega$, so the integrand here has compact support.)

Proof of Theorems 1.3 and 1.5, assuming Proposition 3.7. Theorem 1.3 now follows from Propositions 3.7 and 2.4, using the SOB properties of $\omega_{\mathrm{sf}}(\alpha)$ from Section 3.3. The volume growth is at most 2; as discussed after Proposition 2.4, it therefore was indeed necessary to take care of the integrability condition. Notice that $\left[\omega_{\mathrm{CY}}\right]=[\omega]$ in $H^{2}(M, \mathbb{R})$ because this obviously holds in $H^{2}\left(f^{-1}\left(\mathbb{P}^{1} \backslash \Delta\right), \mathbb{R}\right)$.

The asymptotics in Theorem 1.5 follow from Proposition 2.9. Indeed, parts (ia) and (ib) show that $u \rightarrow \bar{u}$ in $C^{0}$ at the following rates: $\exp (-\delta r)$ in the ALH case, which is $\operatorname{CYL}(0)$ with inj $>0 ; r^{-\delta}$ for ALG (and in fact $r^{-1 / \theta}$ if the cone angle is $\theta$, though we do not need this); $\exp \left(-\delta r^{2 / 3}\right)$ for $\mathrm{I}_{b}$ because the background metric is $\operatorname{CYL}\left(\frac{1}{3}\right)$ with inj decaying only polynomially; $r^{-\delta}$ (in fact, $r^{-2}$ ) for $\mathrm{I}_{b}^{*}$ because the geometry is similar enough to ALG, with inj decaying only logarithmically. By Lemma 2.7, all background metrics are $\operatorname{HMG}(1, k, \alpha)$ for all $k, \alpha$; thus, in the ALH and $\mathrm{I}_{b}$ cases, the derivatives of $u$ decay at the same rate as $u-\bar{u}$, and we even gain a factor of $r^{-1}$ per derivative in the ALG and $\mathrm{I}_{b}^{*}$ cases, by Proposition 2.9)(ii).

In the $\mathrm{I}_{b}$ and $\mathrm{I}_{b}^{*}$ cases, note additionally that $\omega_{\mathrm{CY}}$ satisfies $\operatorname{inj}(x) \sim|B(x, 1)|$ by [9. Theorem 4.3], so this decays at the same rate as for $\omega_{\mathrm{sf}}(\alpha)$. Finally, the bounds on $\left|\nabla^{k} \mathrm{Rm}\right|$ stated in Theorem 1.5(iii) follow from the $\operatorname{HMG}(1, k, \alpha)$ property.

Remark 3.8. (i) The form $\beta$ in Proposition 3.7 is compactly supported in $\Delta^{\prime \prime \prime} \backslash \Delta^{\prime}$. Thus, $\beta=i \partial \bar{\partial} \phi$ with $\phi \sim \log |z|$ on $\Delta^{\prime}$. Since the metric $g$ on $\Delta^{*}$ induced by $g_{\mathrm{sf}}(\alpha)$ is conformal to $|d z|^{2}$, and $g, g_{\mathrm{sf}}(\alpha)$ have the same volume growth, $\phi \circ f$ grows like a Green's function for $g_{0}(\alpha, t)$. Thus, $\left(\omega_{0}(\alpha, t)+i \partial \bar{\partial} u\right)^{2}=\alpha \Omega \wedge \bar{\Omega}$ is in fact solvable for all $\alpha, t \gg 1$, but with $u$ growing like a Green's function in general.

(ii) A bounded harmonic function $u: \Delta^{*} \rightarrow \mathbb{R}$ satisfies $u=\bar{u}+O(|z|)$ for some constant $\bar{u}$, and $r \sim|\log | z||$ in the ALH case, $r \sim|z|^{-\theta}$ in the ALG case with cone 
angle $\theta, r \sim|\log | z||^{3 / 2}$ for $\mathrm{I}_{b}$, and $r \sim|z|^{-1 / 2}|\log | z||^{1 / 2}$ for $\mathrm{I}_{b}^{*}$. The decay estimates from Proposition 2.9 are therefore on the right order of magnitude.

Proof of Proposition 3.7. Write $\Delta(r):=\{|z|<r\} \subset \Delta$. For $0<s<r<\frac{1}{4}$, choose a radial cutoff function $\psi=\psi(r, s), 0 \leq \psi \leq 1$, with $\psi \equiv 1$ on $\Delta(r+s), \operatorname{supp}(\psi) \subset$ $\Delta(r+2 s)$, and $s\left|\psi_{z}\right|+s^{2}\left|\psi_{z \bar{z}}\right| \leq C_{0}$, where here as well as in the rest of the proof, $C_{0}$ stands for a generic constant which is only allowed to depend on $X, \Omega, \omega, \tau_{1}, \tau_{2}$. If a constant may also depend on $r, s$, we write $C_{0}(r, s)$. Even though $r, s$ will only depend on $X, \Omega, \omega, \tau_{1}, \tau_{2}$ eventually, this distinction is needed to avoid a cycle. Fix a $(1,1)$-form $\beta$ on $\mathbb{P}^{1}$ with $\operatorname{supp}(\beta) \subset \Delta(r+3 s) \backslash \Delta(r), 0 \leq \beta \leq|d z|^{2}$, and $\beta=|d z|^{2}$ on $\Delta(r+2 s) \backslash \Delta(r+s)$, identifying $\beta$ with its associated symmetric bilinear form. We also identify $\psi, \beta$ with their pullbacks under $f$ for convenience.

Claim 1 ( $\partial \bar{\partial}$-lemma). There exist a holomorphic section $\tilde{\sigma}$ of $f$ over $\Delta^{*}$ and a smooth function $u_{1}:\left.X\right|_{\Delta^{*}} \rightarrow \mathbb{R}$ such that $T^{*} \omega_{\text {sf }}(1)=\omega+i \partial \bar{\partial} u_{1}$, where $T$ denotes translation by $\tilde{\sigma}$ relative to $\sigma$ as defined in Remark 3.2(i).

Proof. For the case of an $\mathrm{I}_{1}$ singular fiber and $\operatorname{div}(\Omega)=0$, this is Lemma 4.3 in [20]. Nothing essential changes in the general case, but since this proof is quite delicate, we review it here, and give a slightly more direct argument for the last step.

We first need to see why $\left[\omega_{\mathrm{sf}}(1)\right]=[\omega]$ in $H^{2}\left(\left.X\right|_{\Delta^{*}}, \mathbb{R}\right)$. Let $F$ be the homology class of the fibers. Since $\left\langle\omega_{\mathrm{sf}}(1), F\right\rangle=\langle\omega, F\rangle$, and $\langle\omega, C\rangle=0$ (by assumption) and $\left\langle\omega_{\mathrm{sf}}(1), C\right\rangle=0$ (by computation) for all bad cycles $C$, the claim follows if we can show that $H_{2}\left(\left.X\right|_{\Delta^{*}}, \mathbb{Z}\right)$ is spanned by $F$ and by the bad cycles. Retract $\left.X\right|_{\Delta^{*}}$ to a $T^{2}$-bundle $Y$ over $S^{1}=[0,2] / \sim$, and cover $Y$ by $U=\left.Y\right|_{(0,2)}$ and $V=\left.Y\right|_{[0,1) \cup(1,2]}$, so that $U \cap V=\left.\left.Y\right|_{(0,1)} \sqcup Y\right|_{(1,2)}$. Then, from the Mayer-Vietoris sequence,

$$
0 \rightarrow \operatorname{coker}\left(\phi_{2}\right) \rightarrow H_{2}\left(\left.X\right|_{\Delta^{*}}, \mathbb{Z}\right) \rightarrow \operatorname{ker}\left(\phi_{1}\right) \rightarrow 0,
$$

where $\phi_{i}: H_{i}(U \cap V) \rightarrow H_{i}(U) \oplus H_{i}(V)$. Now $\phi_{2}(\alpha, \beta)=(\alpha+\beta, \alpha+\beta)$, and hence $\operatorname{coker}\left(\phi_{2}\right)$ maps onto $\mathbb{Z} F$. Moreover, $\phi_{1}(x, y)=(A x+y, x+y)$, where $A$ denotes the monodromy, so that $\operatorname{ker}\left(\phi_{1}\right)=\{(x,-x): A x=x\}$. By definition, the subgroup of $H_{2}\left(\left.X\right|_{\Delta^{*}}, \mathbb{Z}\right)$ generated by the bad cycles surjects onto this.

Next, if $\zeta$ is a smooth real 1 -form on $\left.X\right|_{\Delta^{*}}$ with $d \zeta$ of type $(1,1)$, then $\xi:=\zeta^{0,1}$ satisfies $\bar{\partial} \xi=0$. From the Leray spectral sequence, $H^{0,1}\left(\left.X\right|_{\Delta^{*}}\right) \cong H^{0}\left(\Delta^{*}, \mathcal{H}\right)$ for the holomorphic line bundle $\mathcal{H}=R^{1} f_{*} \mathcal{O}_{X}$, whose fiber at $z$ is naturally identified with $H^{0,1}\left(f^{-1}(z)\right)$. The fiberwise constant $(0,1)$-form $\Theta:=d \bar{w} / \operatorname{Im}\left(\bar{\tau}_{1} \tau_{2}\right)$ defines a holomorphic section of $\mathcal{H}$. Thus, restricting $\xi$ to $f^{-1}(z)$ and writing the translationinvariant part of this restriction as $s(z) \Theta(z)$ identifies the class of $\xi$ in $H^{0,1}\left(\left.X\right|_{\Delta^{*}}\right)$ with the holomorphic function $s: \Delta^{*} \rightarrow \mathbb{C}$. We apply this to $d \zeta=\omega_{\mathrm{sf}}(1)-\omega$.

We have now rewritten the obstruction against $\omega_{\mathrm{sf}}(1)-\omega$ being $i \partial \bar{\partial}$-exact as a holomorphic function $s: \Delta^{*} \rightarrow \mathbb{C}$. The idea is to kill this obstruction by replacing $\omega_{\mathrm{sf}}(1)$ by $T^{*} \omega_{\mathrm{sf}}(1)$, where $T$ denotes translation by a suitable section. Thus, pick a holomorphic function $\tilde{\sigma}: \Delta^{*} \rightarrow \mathbb{C}$, put $T_{t}(z, w):=(z, w+t \tilde{\sigma}(z))$ for $t \in[0,1]$, and define $T:=T_{1}$. By Cartan's magic formula, $T^{*} \omega_{\mathrm{sf}}(1)-\omega_{\mathrm{sf}}(1)=d \tilde{\zeta}$, where

$$
\tilde{\zeta}=\int_{0}^{1} 2 \operatorname{Re}\left(\tilde{\sigma} \partial_{w}\right)\left\llcorner T_{t}^{*} \omega_{\mathrm{sf}}(1) d t .\right.
$$

One then easily computes that the $(0,1)$-part of $\tilde{\zeta}$ is given by

$$
\tilde{\xi}=\frac{i}{2} \frac{\varepsilon}{\operatorname{Im}\left(\bar{\tau}_{1} \tau_{2}\right)}\left[\tilde{\sigma}(z)(d \bar{w}-\bar{\Gamma}(z, w) d \bar{z})-\frac{1}{2} \bar{\Gamma}(z, \tilde{\sigma}(z)) d \bar{z}\right] .
$$


If we now take $\tilde{\sigma}:=2 i s / \varepsilon$, then the constant part of the restriction of $\xi+\tilde{\xi}$ to any of the torus fibers vanishes, so this form is $\bar{\partial}$-exact by what we said earlier.

We now construct a potential for $T^{*} \omega_{\mathrm{sf}}(\alpha)-\omega$ that depends linearly on $\alpha$. Note that if $\Omega=z^{-(N+1)} k(z) d z \wedge d w$ with $k(0) \neq 0$ as usual, then

$$
\omega_{\mathrm{sf}}(\alpha)-\omega_{\mathrm{sf}}(1)=i(\alpha-1)|k(z)|^{2} \frac{\operatorname{Im}\left(\bar{\tau}_{1} \tau_{2}\right)}{\varepsilon} \frac{d z \wedge d \bar{z}}{|z|^{2 N+2}}=(\alpha-1) i \partial \bar{\partial} u
$$

for some smooth potential $u: \Delta^{*} \rightarrow \mathbb{R}$. This exists simply because $\Delta^{*}$ satisfies the $\bar{\partial}$-Poincaré lemma, though one could also write down an explicit potential $u$ based on [20, p. 486]. We identify $u$ with $u \circ f$. Thus, with $T, u_{1}$ from Claim 1 ,

$$
T^{*} \omega_{\mathrm{sf}}(\alpha)=\omega+i \partial \bar{\partial} u_{\alpha}, \quad u_{\alpha}:=u_{1}+(\alpha-1) u .
$$

Notice that $u_{1}$ and $u$ are not unique, but we make a fixed choice for both of them once and for all. Then $C_{0}$ is allowed to depend on these choices as well.

It will turn out to be necessary below to normalize $u$ by a harmonic function in the gluing region $\Delta(r+2 s) \backslash \Delta(r+s)$ to obtain more specific control there. Then the $(r, s)$-dependence of this correction needs to be tracked carefully.

Claim 2 (normalizing the potential). If $C_{0} r<1, C_{0} s<r$, and $v$ is harmonic with the same boundary values as $u$ on $\Delta(r+3 s) \backslash \Delta(r)$, then

$$
\sup _{\Delta(r+2 s) \backslash \Delta(r+s)}\left(s^{-2}|u-v|+s^{-1}\left|(u-v)_{z}\right|\right) \leq C_{0} \sup _{\Delta(r+3 s) \backslash \Delta(r)} u_{z \bar{z}} .
$$

Proof. Put $w:=u-v$ and take $b(z):=(|z|-r)(r+3 s-|z|)$ as a barrier. If $3 s \leq r$, then $4 b_{z \bar{z}} \leq-1$; thus $\sup |w| \leq 4(\sup |b|)\left(\sup \left|w_{z \bar{z}}\right|\right)=9 s^{2} \sup u_{z \bar{z}}$ by the maximum principle, where all suprema are taken over $\Delta(r+3 s) \backslash \Delta(r)$.

The derivative estimate in (3.22) now follows from local elliptic regularity on the pairs of disks $B\left(z, \frac{1}{2} s\right) \subset B\left(z, \frac{3}{2} s\right)$ with $|z|=r+\frac{3}{2} s$, together with the key fact that $s\left|u_{z \bar{z}, z}\right| \leq C_{0} u_{z \bar{z}}$ on $\Delta(r+3 s) \backslash \Delta(r)$ from (3.20) and Table 1.

We now construct the desired complete background Kähler metric $\omega_{0}$. For every $\alpha>0$ and $t>0$, define a closed $(1,1)$-form $\omega_{0}(\alpha, t)$ on $M$ by setting

$$
\begin{aligned}
& \omega_{0}(\alpha, t):= \begin{cases}\omega+t \beta+i \partial \bar{\partial}\left(\psi \tilde{u}_{\alpha}\right) & \text { outside }\left.X\right|_{\Delta(r)}, \\
\omega+t \beta+i \partial \bar{\partial} u_{\alpha} & \text { over } \Delta(r+s)^{*},\end{cases} \\
& \tilde{u}_{\alpha}:=u_{1}+(\alpha-1)(u-v) \text { on } \Delta(r+3 s) \backslash \Delta(r),
\end{aligned}
$$

where we identify $v$ with $v \circ f$. The definitions match over $\Delta(r+s) \backslash \Delta(r)$ because $\psi \equiv 1$ there and $u_{\alpha}-\tilde{u}_{\alpha}=(\alpha-1) v$, which is pluriharmonic. Notice that

$$
\omega_{0}(\alpha, t)= \begin{cases}\omega & \text { outside }\left.X\right|_{\Delta(r+3 s)}, \\ T^{*} \omega_{\mathrm{sf}}(\alpha) & \text { over } \Delta(r)^{*} .\end{cases}
$$

Claim 3 (positivity of the glued form). There exists a constant $C_{0}(r, s)>0$ such that if $t>C_{0}(r, s)+C_{0}|\alpha-1| \sup _{\Delta(r+3 s) \backslash \Delta(r)} u_{z \bar{z}}$, then, on all of $M$,

$$
\omega_{0}(\alpha, t) \geq \frac{1}{2}\left(\omega+\psi i \partial \bar{\partial} u_{\alpha}\right)>0 .
$$

Proof. This only needs to be checked over $\Delta(r+2 s) \backslash \Delta(r+s)$. We calculate

$$
\begin{aligned}
\omega_{0}(\alpha, t)= & \omega+t \beta+\psi i \partial \bar{\partial} u_{\alpha}+\left[\psi_{z} u_{1, \bar{w}} i d z \wedge d \bar{w}+\psi_{\bar{z}} u_{1, w} i d w \wedge d \bar{z}\right. \\
& \left.+\left\{\psi_{z \bar{z}} \tilde{u}_{\alpha}+\psi_{\bar{z}} \tilde{u}_{\alpha, z}+\psi_{z} \tilde{u}_{\alpha, \bar{z}}\right\} i d z \wedge d \bar{z}\right] .
\end{aligned}
$$


Since $\omega+i \partial \bar{\partial} u_{\alpha}=T^{*} \omega_{\text {sf }}(\alpha)>0$ over $\Delta^{*}$, we have $\omega+\psi i \partial \bar{\partial} u_{\alpha}>0$ by convexity. To compensate for the $d z \wedge d \bar{w}$ and $d w \wedge d \bar{z}$ terms, we note that

$$
(z, w)\left(\begin{array}{cc}
0 & a \\
\bar{a} & 0
\end{array}\right)\left(\begin{array}{c}
\bar{z} \\
\bar{w}
\end{array}\right)=2 \operatorname{Re}(a z \bar{w}) \geq-\frac{|a|}{\delta}|z|^{2}-\delta|a||w|^{2} \quad(a \in \mathbb{C}, \delta>0) .
$$

We apply this by choosing $\delta$ so that $2 \delta \sup \left|\psi_{z} u_{1, \bar{w}}\right|=\inf \left(\omega+\psi i \partial \bar{\partial} u_{1}\right)_{w \bar{w}}$, the sup and inf being taken over $\Delta(r+2 s) \backslash \Delta(r+s)$, and then making $t>C_{0}(r, s) \gg 1$. To treat the $d z \wedge d \bar{z}$ terms, consider (3.24): We can absorb the $u_{1}$ part by making $C_{0}(r, s)$ larger, and we use (3.22) to deal with the $(\alpha-1)(u-v)$ contribution.

Thus, given any $\alpha>0$, if $t$ is large enough as specified in Claim 3 , then $\omega_{0}(\alpha, t)$ defines a complete Kähler metric on $M$, which by (3.23) and (3.25) satisfies all the required properties except that $\omega_{0}(\alpha, t)^{2}-\alpha \Omega \wedge \bar{\Omega} \in C_{0}^{\infty}\left(\wedge^{4} M\right)$ will not have zero mass in general, as needed for the integrability condition.

Before addressing this final point, let us agree that the constants $C_{0}$ and $C_{0}(r, s)$ are now fixed once and for all (with $C_{0}$ independent of $r, s$ ). We then also require a new generic constant, $\bar{C}_{0}$, which is still allowed to change from line to line, but is again understood to be independent of $r$ and $s$.

Claim 4 (integrability condition). One can choose $r, s$, depending on $X, \Omega, \omega$, $\tau_{1}, \tau_{2}$, such that for all $t^{\prime}>1$ there exists precisely one $\alpha>0$ such that $I(\alpha, t):=$ $\int\left(\omega_{0}(\alpha, t)^{2}-\alpha \Omega \wedge \bar{\Omega}\right)=0$ with $t:=C_{0}(r, s) t^{\prime}+C_{0}|\alpha-1| \sup _{\Delta(r+3 s) \backslash \Delta(r)} u_{z \bar{z}}$.

The key point to note here is that $I(\alpha, t)$ is linear in both variables, from (3.23). Thus, Proposition 3.7 follows immediately from what is established in Claim 4.

In the following proof, it is helpful to bear in mind that $\alpha$ may always be chosen as small or as large as necessary, depending on $r, s, t^{\prime}$. It is very important though that all upper bounds on contributions to $I(\alpha, t)$ are at worst of the form $\bar{C}_{0} \alpha$.

Proof. Let $r, s>0$ be arbitrary for now, but with $C_{0} r<1, C_{0} s<r$. For any given $t^{\prime}>1$ and $\alpha>0$, define $t$ as in the claim. We will show that $r, s$ can be chosen so that $I(\alpha, t)>0$ if $\alpha$ is small and $I(\alpha, t)<0$ if $\alpha$ is large (exactly how small or large is irrelevant). We begin by observing that it suffices to integrate over $\left.X\right|_{\mathbb{P}^{1} \backslash \Delta(r)}$ in the definition of $I(\alpha, t)$. Then, for the first direction, notice that

$$
\int_{\left.X\right|_{\mathbb{P} 1 \backslash \Delta(r)}} \omega_{0}(\alpha, t)^{2} \geq \int_{\left.X\right|_{\mathbb{P} 1} \backslash \Delta(r+3 s)} \omega^{2},
$$

so $I(\alpha, t)>0$ if $\alpha$ is small enough, depending on $\Omega, \omega, r, s$. For the other direction, we can assume that $\alpha>1+C_{0}(r, s) t^{\prime}$. Then, over $\Delta(r+3 s) \backslash \Delta(r)$,

$$
\omega_{0}(\alpha, t) \leq 2\left(\omega+\psi i \partial \bar{\partial} u_{1}\right)+(\alpha-1) i \partial \bar{\partial} u+2 t \beta ;
$$

this follows from estimates as in the proof of Claim 3 , and from the definition of $t$. Now notice that $2 t \beta \leq \bar{C}_{0}(\alpha-1) i \partial \bar{\partial} u$ because $\beta \leq C_{0} i \partial \bar{\partial} u$ and

$$
\sup _{\Delta(r+3 s) \backslash \Delta(r)} u_{z \bar{z}} \leq C_{0} \inf _{\Delta(r+3 s) \backslash \Delta(r)} u_{z \bar{z}} ;
$$

the point is that $u_{z \bar{z}} \sim|z|^{a}|\log | z||^{b} \rightarrow \infty$ as $|z| \rightarrow 0$ from (3.20) and Table 1. Thus, using $(\psi-1) i \partial \bar{\partial} u_{1} \leq \omega$ from Claim 1, and then (3.21),

$$
\omega_{0}(\alpha, t) \leq 2 \omega+\bar{C}_{0} T^{*} \omega_{\text {sf }}(\alpha)
$$

over $\Delta(r+3 s) \backslash \Delta(r)$. Since all forms in sight are positive, this implies that

$$
\omega_{0}(\alpha, t)^{2} \leq \bar{C}_{0}\left(\omega^{2}+\omega \wedge T^{*} \omega_{\mathrm{sf}}(\alpha)+\alpha \Omega \wedge \bar{\Omega}\right)
$$


on the same region. Bearing in mind (3.25) and that $\int \omega^{2}<\infty$ by assumption, and writing $X_{r, s}=\left.X\right|_{\Delta(r+3 s) \backslash \Delta(r)}$ and $X_{r}=\left.X\right|_{\mathbb{P}^{1} \backslash \Delta(r)}$, we obtain

$$
\begin{gathered}
I(\alpha, t) \leq \bar{C}_{0}\left(1+I_{1}+\alpha I_{2}\right)-\alpha I_{3}, \\
I_{1}=\int_{X_{r, s}} \omega \wedge T^{*} \omega_{\mathrm{sf}}(\alpha), \quad I_{2}=\int_{X_{r, s}} \Omega \wedge \bar{\Omega}, \quad I_{3}=\int_{X_{r}} \Omega \wedge \bar{\Omega} .
\end{gathered}
$$

We have $I_{3} \rightarrow \infty$ as $r \rightarrow 0$. For any fixed $r$, we also have $I_{2} \rightarrow 0$ as $s \rightarrow 0$. Finally, using (3.21) and $\alpha>1$, we find that for any fixed $r, \delta>0$ there exists $s_{r, \delta}>0$ such that $s<s_{r, \delta}$ implies $I_{1}<\delta \alpha$. Thus, $I(\alpha, t)<0$ if $r \ll 1, s \ll r$, and $\alpha \gg 1$.

\section{UNIQUENESS AND MODULI IN THE CYLINDRICAL ENDS CASE}

We now consider ALH hyper-Kähler manifolds $\left(M^{4}, g\right)$, where $M$ is diffeomorphic to the complement of a smooth fiber, $D$, in a rational elliptic surface $X$. However, we will not assume that $M$ with a $g$-parallel complex structure is biholomorphic to $X \backslash D$, or even that $g$ is obtained from Theorem 1.3. In fact, our main goal here is to make some first steps towards the obvious classification questions.

4.1. Hodge theory on ALH spaces and consequences. We begin by recalling well-known facts for manifolds with cylindrical ends, specialized to $M$. Our source is Nordström's nice survey in 47, which has references to the original papers.

Since $\pi_{1}(M)=0$ and $H^{2}(X)=H^{1,1}(X)=\mathbb{R}^{10}$, the Mayer-Vietoris sequence

$$
0 \rightarrow H^{1}\left(T^{2}\right) \rightarrow H^{1}\left(T^{3}\right) \rightarrow H^{2}(X) \rightarrow H^{2}(M) \oplus H^{2}\left(T^{2}\right) \rightarrow H^{2}\left(T^{3}\right) \rightarrow 0
$$

yields $H^{2}(M)=\mathbb{R}^{11}$. More precisely, the restriction map $H^{2}(X) \rightarrow H^{2}(M)$ has a 1-dimensional kernel (spanned by the Poincaré dual of the fibers, i.e. $\left.c_{1}(X)\right)$ and maps onto the 9-dimensional subspace $H^{1,1}(M) \subset H^{2}(M)$ of classes containing a closed form which is $(1,1)$ with respect to the complex structure of $X$.

Next, we have $H_{c}^{2}(M)=\mathbb{R}^{11}$ and $\operatorname{im}\left(H_{c}^{2}(M) \rightarrow H^{2}(M)\right)=\mathbb{R}^{8}$ by the long exact sequence for $(X \backslash U, \partial U)$, where $U \supset D$ is a tubular neighborhood. Recall that the classes in the image are precisely the ones that contain compactly supported forms. If $g$ is any ALH Riemannian metric on $M$, then these classes are in bijection with the $L^{2}$-harmonic 2 -forms with respect to $g$. If $g$ is in addition Kähler with Ric $\geq 0$, then the $L^{2}$-harmonics are primitive $(1,1)$ since $\left[\Delta_{d}, \omega \wedge-\right]=0$ and because harmonic ED $(2,0)$-forms are trivial by the Bochner formula; if $g$ is even Kähler with respect to the complex structure of $X$, then $H^{1,1}(M)=\mathbb{R}[\omega] \oplus L^{2}$-harmonics.

Back to a general ALH metric, the space of bounded $\Delta_{d}$-harmonic $r$-forms with respect to $g$ has dimension $\operatorname{dim} H^{r}(M)+\operatorname{dim} H_{c}^{r}(M)-\operatorname{dim} \operatorname{im}\left(H_{c}^{r}(M) \rightarrow H^{r}(M)\right)$. Outside a compact set, any such form can be written as the sum of a $g$-parallel and an ED form. Thus, the space of bounded $\Delta_{d}$-harmonic 2-forms splits (not uniquely) as the direct sum of its 8-dimensional subspace in $L^{2}$, and a 6 -dimensional subspace of forms asymptotic to $\alpha \wedge d t+\beta$, where all $\alpha \in \wedge^{1} \mathbb{R}^{3}$ and $\beta \in \wedge^{2} \mathbb{R}^{3}$ occur.

Finally, we will need to know that $\Delta_{d}$ is Fredholm of index $-\left(b_{r}\left(T^{3}\right)+b_{r-1}\left(T^{3}\right)\right)$ when acting on weighted Hölder spaces of $r$-forms with a small enough exponential decay rate; also, there are elliptic estimates for $\Delta_{d}$ acting on such spaces.

Proposition 4.1. Let $\beta$ be an ED $r$-form on $M$ with $r \in\{0,1,2\}$.

(i) If $r=0$, then for any fixed ALH coordinate system there exists a unique $\alpha=$ $C t+\mathrm{ED}$ such that $\Delta_{d} \alpha=\beta$, and moreover $C=0$ if and only if $\int \beta d \mathrm{vol}=0$. 
(ii) If $r=1$, there exists a unique $\alpha=$ parallel $+\mathrm{ED}$ with $\Delta_{d} \alpha=\beta$. The parallel part of $\alpha$ vanishes if and only if $\int\langle\beta, \eta\rangle d \mathrm{vol}=0$ for all $\Delta_{d}$-harmonic 1 -forms $\eta$ of linear growth. The space of all such harmonic 1-forms has dimension 4 .

(iii) If $r=2$, then a smooth and in addition $\mathrm{ED} 2$-form $\alpha$ with $\Delta_{d} \alpha=\beta$ exists if and only if $\int\langle\beta, \eta\rangle d \mathrm{vol}=0$ for all bounded $\Delta_{d}$-harmonic 2-forms $\eta$.

Proof. (i) The condition $\int \beta=0$ is necessary for an ED solution $\alpha$ to exist, hence sufficient because the index is -1 and the kernel is trivial. Every function $\alpha_{0}$ with $\alpha_{0}=t+\mathrm{ED}$ at infinity satisfies $\Delta_{d} \alpha_{0}=\mathrm{ED}$, but if $\Delta_{d} \alpha_{0}=0$, then $d \alpha_{0}$ is bounded $\Delta_{d}$-harmonic; hence $\alpha_{0}=0$. Thus, $\Delta_{d}: \operatorname{ED} \oplus \mathbb{R} \alpha_{0} \rightarrow \mathrm{ED}$ is surjective.

(ii) The index of $\Delta_{d}$ on ED 1-forms is -4 , and we can obviously find four linearly independent 1-forms that are parallel + ED outside a compact set, whereas, again, there are no bounded $\Delta_{d}$-harmonic 1 -forms at all. The existence claim follows. In turn, this shows that we can correct any 1 -form which is equal to $t \xi+\mathrm{ED}$ outside a compact set for some constant 1 -form $\xi$ by a bounded 1-form so as to become $\Delta_{d^{-}}$ harmonic. This yields a 4-dimensional space of linearly growing harmonic 1-forms, which must define the cokernel of $\Delta_{d}$ on ED 1-forms because the index is -4 .

(iii) The given condition is clearly necessary again, so that dim coker $\geq 14$. On the other hand, we know that the index is -6 and that dim ker $=8$.

Remark 4.2. On manifolds asymptotic to $\mathbb{R}^{+} \times Y$, the space of bounded harmonic $r$-forms modulo ED always has dimension $\leq b_{r}(Y)+b_{r-1}(Y)$. It is a key point for us (and rather a coincidence) that we have equality for $r=2$ in our setting.

We now discuss two fairly immediate applications of this analysis. The first one is the proof of Theorem [1.9 this easily generalizes to higher dimensions.

Proof of Theorem 1.9. Define $\omega:=\Psi^{*} \omega_{2}-\omega_{1}$. From our assumptions, $\omega=$ ED on $M_{1}$. By Proposition 4.1(ii), there exists a bounded 1-form $\alpha=$ parallel + ED such that $\Delta_{d} \alpha=d^{*} \omega$. Since $\omega$ is exact by assumption, $d \alpha-\omega$ is $\Delta_{d}$-harmonic, ED, and exact; hence $d \alpha=\omega$ by the Hodge theorem. Now $\bar{\partial}^{*} \alpha^{0,1}$ is an ED function. Thus, by Proposition 4.1(i) and the Kähler identities, there exists a function $\gamma$ of at most linear growth such that $\bar{\partial}^{*} \bar{\partial} \gamma=\bar{\partial}^{*} \alpha^{0,1}$. Since $\bar{\partial} \alpha^{0,1}=0$ for type reasons, and again by the Kähler identities, $\bar{\partial} \gamma-\alpha^{0,1}$ is a bounded $\Delta_{d}$-harmonic 1 -form, hence equal to zero. Thus, eventually, $\omega=i \partial \bar{\partial} u$, where $u:=2 \operatorname{Im}(\gamma)=$ const $\cdot t+\mathrm{ED}$.

Now $\Psi^{*} \omega_{2}^{2}=h \omega_{1}^{2}$ with $h=1+\mathrm{ED}$, but in fact $h \equiv 1$ because both $\Psi^{*} \omega_{2}$ and $\omega_{1}$ are Calabi-Yau, so that the associated holomorphic 2-forms $\Psi^{*} \Omega_{2}=f \Omega_{1}$, where $f$ is holomorphic with $|f|^{2}=h=1+$ ED and hence $f \equiv$ const. Since $\omega=i \partial \bar{\partial} u$, this implies that $\Delta u=0$, where the Laplacian is taken with respect to the metric $g_{1}+\Psi^{*} g_{2}$. This metric is still ALH, so that $u=0$, and hence $\omega=0$, as desired.

Our second application is the unobstructedness of ALH complex deformations. This proof is fairly specific to the case at hand, somewhat in analogy with how the unobstructedness of $K 3$ surfaces was proved long before [51, 55] were available. We will use standard ideas from deformation theory; cf. [4, §12.J] and [26, §6].

Corollary 4.3. Let $\left(g_{0}, J_{0}, \Omega_{0}\right)$ be an ALH Calabi-Yau structure on $M$. Let I be a bounded $T^{1,0} M$-valued $(0,1)$-form with $\bar{\partial} I=\bar{\partial}^{*} I=0$ that is $g_{0}$-selfadjoint as an endomorphism of TM. Then there exists a family $\left(g_{s}, J_{s}\right)$ of ALH Kähler structures with holomorphic volume forms $\Omega_{s}$ such that $g_{s}, J_{s}, \Omega_{s} \in L^{\infty}$ and $\left.\frac{d}{d s}\right|_{s=0} J_{s}=I$. 
Proof. We first integrate $I$ as a deformation of the complex structure. For this, it suffices to find a family $I_{s}=I s+O\left(s^{2}\right)$ of bounded $T^{1,0} M$-valued $(0,1)$-forms such that $\bar{\partial} I_{s}+\left[I_{s}, I_{s}\right]=0$, where $[-,-]$ denotes the usual Frölicher-Nijenhuis bracket. Indeed, if we then view $I_{s}$ as an endomorphism of $T M$ that anticommutes with $J_{0}$, the almost-complex structures $J_{s}:=J_{0}\left(1-I_{s}\right)\left(1+I_{s}\right)^{-1}$ (which are characterized by the fact that the $(0,1)$-space with respect to $J_{s}$ can be written as $\left\{v^{\prime \prime}+\left(I_{s} v\right)^{\prime}\right.$ : $v \in T M\}$, where $v^{\prime}=v-i J_{0} v$ and $\left.v^{\prime \prime}=v+i J_{0} v\right)$ are honest complex structures by Newlander-Nirenberg, and we obviously have $J_{s}=J_{0}+2 I J_{0} s+O\left(s^{2}\right)$.

Following the classical approach in the compact case [30, we will construct the solution as a power series $I_{s}=\sum_{k=1}^{\infty} I_{k} s^{k}$, where $I_{1}=I$ and all $I_{k}$ with $k \geq 2$ are ED. Notice that the Frölicher-Nijenhuis bracket of constant forms on a flat cylinder vanishes. Thus, to build up a formal power series solution order by order, we must solve the following problem: Given a $T^{1,0} M$-valued $\operatorname{ED}(0,2)$-form $L$, find a $T^{1,0} M$ valued ED $(0,1)$-form $K$ such that $\bar{\partial} K=L$. Dualizing with $\Omega_{0}$, it suffices to solve $\bar{\partial} \alpha=\beta$ with $\alpha=\mathrm{ED}$ if $\beta=\mathrm{ED}$ is a given $(1,2)$-form. Now Proposition 4.1(iii) tells us that there exists an ED $(1,1)$-form $\alpha$ such that $\Delta_{\bar{\partial}} \alpha=\bar{\partial}^{*} \beta$. Then both $\bar{\partial}^{*} \alpha$ and $*(\bar{\partial} \alpha-\beta)$ are ED harmonic 1 -forms, so that $\bar{\partial}^{*} \alpha=0$ and $\bar{\partial} \alpha=\beta$. Convergence of the formal power series then follows from elliptic estimates as in 30.

Next, we show that there exists a family $\omega_{s}$ of $J_{s}$-Kähler metrics that are ALH. The classical Kähler stability theorem in the compact case 31. seems very hard to generalize, so we instead adopt a power series approach inspired by [16.

Let $\mathfrak{P}_{s}^{p, q}$ denote the projection onto $(p, q)$-forms with respect to $J_{s}$. We aim to construct the desired ALH Kähler form as $\omega_{s}=\mathfrak{P}_{s}^{1,1} \sum_{k=0}^{\infty} \omega_{k} s^{k}$ with $\omega_{k}$ of $J_{0}$-type $(1,1)$ and ED if $k \geq 1$. Then all we need to make sure is that $d \omega_{s}=0$. Decomposing this condition order by order shows that we must solve $d \omega_{k}=d \eta_{k}$ for $\omega_{k}$ of $J_{0}$-type $(1,1)$, where $\eta_{k}$ is a real 2 -form depending on $\omega_{0}, \ldots, \omega_{k-1}$ and $J_{0}, \ldots, J_{k}$ in a specific fashion, but with no control on its type; here, $J_{s}=\sum_{k=0}^{\infty} J_{k} s^{k}$ denotes the power series from above. The key point now is that $\eta_{1}=\mathrm{ED}$, as opposed to merely $O(1)$, because $I$ is $g_{0}$-selfadjoint. Thus, we obtain an initial solution

$$
\omega_{1}:=\mathfrak{P}_{0}^{1,1} \eta_{1}-\left(\partial_{0} \alpha_{1}+\bar{\partial}_{0} \bar{\alpha}_{1}\right)=\mathrm{ED},
$$

where $\alpha_{1}$ is the unique bounded $(0,1)$-form with $\bar{\partial}_{0} \alpha_{1}=\mathfrak{P}_{0}^{0,2} \eta_{1}$ and $\bar{\partial}_{0}^{*} \alpha_{1}=0$, to be constructed using Proposition 4.1(ii) and the fact that there are no nonzero ED harmonic $(0,2)$-forms. We then proceed inductively, replacing $\eta_{1}$ in (4.1) by $\eta_{k}$; it is again standard to obtain convergence from elliptic estimates, as in 30.

Given the ALH Kähler metrics $\omega_{s}$, fitting the holomorphic volume form $\Omega_{0}$ into a family is easy now: We can simply take $\Omega_{s}:=\mathfrak{P}_{s}^{2,0} \Omega-\alpha_{s}$, where $\alpha_{s}$ denotes the unique ED $J_{s}-(2,0)$-form that satisfies $\bar{\partial}_{s} \alpha_{s}=\bar{\partial}_{s} \mathfrak{P}_{s}^{2,0} \Omega$, which exists and depends smoothly on $s$ by Proposition 4.1(iii) and the underlying elliptic theory.

4.2. The kernel of the linearized Einstein operator. For a hyper-Kähler ALH manifold $\left(M^{4}, g\right)$ as before, we now describe the kernel, $\mathcal{E}$, of the linearized Einstein operator inside the space $\mathcal{H}$ of all symmetric bilinear forms that are parallel + ED outside a compact set. Kovalev [35] already indicated how to do this for Calabi-Yau manifolds with cylindrical ends in general, generalizing Koiso's approach [33] in the compact case, but his description of the kernel is not accurate; cf. Remark 4.6.

The integrability of the infinitesimal Einstein deformations could then be proved in several ways: Given Corollary 4.3 , it is not hard to extend Koiso's proof; compare 
[22, 35] and Remark 4.5)(i). Another option is to reduce to the $G_{2}$-theory from [4]. All in all, we will thus have completed the proof of Theorem 1.10 .

In order to give a careful description of the kernel, we proceed in three steps.

- Study the deformations of the flat metric on $\mathbb{R} \times T^{3}$ from Koiso's perspective.

- Prove that $\mathcal{E}=\mathcal{D} \oplus \mathcal{L}_{\text {bd }}$, where $\mathcal{D}=$ ker $\Delta_{\text {Lich }}$ has dimension 34 and admits a clear geometric description, whereas $\mathcal{L}_{\text {bd }}=\left\{L_{X} g: X=\right.$ parallel + ED $\}$.

- Identify those Lie derivatives of $g$ that are still contained in $\mathcal{D}$. This turns out to be the 4-dimensional space $\mathcal{L}_{\text {lin }}=\left\{L_{X} g: X\right.$ linearly growing harmonic $\}$.

Step 1: Deformations of flat cylinders. Equip $\mathbb{C}^{2}$ with its standard Kähler structure $(g, J)$ and let $\Lambda \subset \mathbb{C}^{2}$ be a rank-3 lattice. Then $\mathbb{C}^{2} / \Lambda \cong \mathbb{R} \times T^{3}$ for the flat metric on $T^{3}=\mathbb{R}^{3} / \mathbb{Z}^{3}$ induced by any marking $\mathbb{Z}^{3} \rightarrow \Lambda$. Hence, the deformations of the flat metric on $\mathbb{C}^{2} / \Lambda$, including a scale, are naturally identified with $\mathrm{GL}(3, \mathbb{R}) / \mathrm{O}(3)$. We now compare this with Koiso's point of view; see [33 and [4, §12.J].

The natural space of infinitesimal Ricci-flat deformations here is $\mathcal{D}=\mathrm{Sym}^{2} \mathbb{R}^{4}$. We decompose $\mathcal{D}$ into its $J$-Hermitian and $J$-skew-Hermitian parts, $\mathcal{D}=\mathcal{D}^{+} \oplus \mathcal{D}^{-}$. Writing $h(u, v)=g(H u, v)$ for $h \in \mathcal{D}$, we then introduce a linear map

$$
\mathfrak{I}: \mathcal{D} \rightarrow \mathbb{R}^{4 \times 4}, \quad \mathfrak{I}(h):=H^{+} J+H^{-} .
$$

This defines an isomorphism of $\mathrm{U}(2)$-modules between $\mathcal{D}$ and $\mathfrak{s p}(4, \mathbb{R})$ :

$$
\begin{aligned}
& \Im\left(\mathcal{D}^{+}\right)=\left\{I \in \mathbb{R}^{4 \times 4}: I^{\mathrm{tr}}+I=I J-J I=0\right\}=\mathfrak{u}(2), \\
& \Im\left(\mathcal{D}^{-}\right)=\left\{I \in \mathbb{R}^{4 \times 4}: I^{\mathrm{tr}}-I=I J+J I=0\right\} \cong \mathfrak{s p}(4, \mathbb{R}) / \mathfrak{u}(2) .
\end{aligned}
$$

We can view $\mathfrak{I}\left(\mathcal{D}^{-}\right)$as a space of infinitesimal deformations of $J$. In general, if $I \in \mathbb{R}^{4 \times 4}$ with $|I|<1$ satisfies $I J+J I=0$, then we have a canonically associated complex structure $\mathfrak{C}(I)$ on $\mathbb{R}^{4}$ whose $(0,1)$-space in $\mathbb{R}^{4} \otimes \mathbb{C}$ is equal to $\left\{u^{\prime \prime}+(I u)^{\prime}\right.$ : $\left.u \in \mathbb{R}^{4}\right\}$, where $u^{\prime}=u-i J u$ and $u^{\prime \prime}=u+i J u$. Explicitly,

$$
\mathfrak{C}(I)=J(1-I)(1+I)^{-1} .
$$

(Recall that we already used this transformation in the proof of Corollary 4.3.) If in addition $I^{\text {tr }}=I$, or in other words, $I \in \mathfrak{I}\left(\mathcal{D}^{-}\right)$, then $\omega(u, v)=g(J u, v)$ is still $(1,1)$ with respect to $\mathfrak{C}(I)$; more precisely, $\mathfrak{C}$ identifies its bounded domain of definition in $\mathfrak{I}\left(\mathcal{D}^{-}\right)$with the Siegel upper half-plane

$$
\mathfrak{H}_{2}=\operatorname{Sp}(4, \mathbb{R}) / \mathrm{U}(2)=\left\{K \in \mathbb{R}^{4 \times 4}: K^{2}=-1, K^{*} \omega=\omega\right\},
$$

and is in fact nothing else but the Cayley transform associated with this Hermitian symmetric space. By contrast, $\mathfrak{C}$ is globally defined on the complementary subspace of all $I \in \mathbb{R}^{4 \times 4}$ such that $I J+J I=0$ and $I^{\mathrm{tr}}+I=0$, which is a complex line, and maps this to the complement of $-J$ in the twistor space $\left(\cong \mathbb{P}^{1}\right)$ of $\mathbb{C}^{2}$.

We conclude with two observations that will be helpful later on for interpreting the kernel of the Lichnerowicz Laplacian on a Ricci-flat ALH space.

(i) $\mathcal{D}$ has dimension 10 and hence overcounts the number of moduli of the flat metric $g$ on $\mathbb{C}^{2} / \Lambda$ by 4 . This is because $\mathcal{D}$ contains a 4 -dimensional space $\mathcal{L}_{\text {lin }}:=$ $\{h \in \mathcal{D}: H(\Lambda)=0\}$ of Lie derivatives of $g$ by linear vector fields on $\mathbb{C}^{2} / \Lambda$.

(ii) Suppose the cross section $T^{3}=S^{1} \times T^{2}$ is an isometric product. Then

$$
\Lambda=\mathbb{Z}\left(2 \pi \ell e_{2}\right)+\Lambda^{\prime}, \quad \Lambda^{\prime}=\frac{\varepsilon}{\operatorname{Im}(\tau)}(\mathbb{Z}+\mathbb{Z} \tau) \subset \mathbb{C}=\mathbb{R} e_{3} \oplus \mathbb{R} e_{4},
$$


with $\varepsilon, \ell>0$ and $\tau \in \mathfrak{H}$, after rotating $\Lambda$ if necessary. Modulo $\mathcal{L}_{\text {lin }}$, we therefore have 2 skew-Hermitian moduli dual to $\tau$, and 4 Hermitian moduli. One of these is a scale, one is dual to $\ell^{2} / \varepsilon$, and the other two break the isometric splitting.

Remark 4.4. Step 1(ii) shows that if $\Lambda$ isometrically splits off a $\mathbb{Z}$-factor, then even though this splitting can be deformed away, the complex structure on the cylinder nevertheless remains compactifiable as $\mathbb{P}^{1} \times\left(\mathbb{C} / \Lambda^{\prime}\right)$ after a hyper-Kähler rotation.

Step 2: The kernel of the linearized Einstein operator. We will be following [35] to some extent. To begin with, recall that the linearization, $\mathfrak{E}$, of the Ricci curvature at $g$ is given by $2 \mathfrak{E} h=\nabla^{*} \nabla h-2 \mathfrak{R} h+L_{\mathfrak{B} h} g$, where $\mathfrak{R} h=\sum h\left(R\left(E_{i},-\right)-, E_{i}\right)$ and the Bianchi operator $\mathfrak{B} h=\operatorname{div}\left(h-\frac{1}{2}(\operatorname{tr} h) g\right)^{\sharp}$. Our goal in this step is to describe the space $\mathcal{E}=\left.\operatorname{ker} \mathfrak{E}\right|_{\mathcal{H}}$ as explicitly as possible.

We begin by recalling the identity $\mathfrak{B}\left(L_{X} g\right)=-\nabla^{*} \nabla X+\operatorname{Ric} X$, which holds for all Riemannian metrics $g$ and vector fields $X$. Using this and Proposition4.1(ii), we then find that, in our situation, any given $h \in \mathcal{H}$ can be corrected by a Lie derivative $L_{X} g$ with $X=$ parallel + ED outside a compact set such that $\mathfrak{B}\left(h+L_{X} g\right)=0$. We denote the space of all such Lie derivatives by $\mathcal{L}_{\mathrm{bd}}$. Thus,

$$
\mathcal{E}=\left(\left.\mathcal{D} \cap \operatorname{ker} \mathfrak{B}\right|_{\mathcal{H}}\right) \oplus \mathcal{L}_{\text {bd }}, \quad \mathcal{D}:=\left\{h \in \mathcal{H}: \nabla^{*} \nabla h-2 \mathfrak{R} h=0\right\}
$$

the two spaces are transversal because otherwise $M$ would admit a nonzero vector field $X=$ parallel + ED with $\nabla^{*} \nabla X=0$, so that $\nabla X=0$ and $M$ would split off a line. The space $\mathcal{D}=$ ker $\Delta_{\text {Lich }}$ is finite-dimensional; note that we have chosen the same symbol $\mathcal{D}$ as in Step 1 because this space is the exact counterpart to $\mathrm{Sym}^{2} \mathbb{R}^{4}$ over $\mathbb{C}^{2} / \Lambda$. We will continue to use notation analogous to Step 1.

Fix a parallel orthogonal almost-complex structure $J$. Then $\mathcal{D}=\mathcal{D}^{+} \oplus \mathcal{D}^{-}$, and there are well-known Bochner formulas [4, §12.J] which show that $\mathfrak{I}\left(\mathcal{D}^{+}\right)$is equal to the space of bounded $\Delta_{d}$-harmonic real $(1,1)$-forms, and $\Im\left(\mathcal{D}^{-}\right)$is equal to the space of bounded $\Delta_{\bar{\partial}}$-harmonic $T^{1,0} M$-valued $(0,1)$-forms which are $g$-selfadjoint as endomorphisms of $T M$. By dualizing with the holomorphic symplectic form $\Omega$, $\Delta_{\bar{\partial}}$-harmonic $T^{1,0} M$-valued $(0,1)$-forms are identified with $\Delta_{d}$-harmonic $\mathbb{C}$-valued $(1,1)$-forms; note in particular that selfadjoint $T^{1,0} M$-valued $(0,1)$-forms map to primitive $\mathbb{C}$-valued $(1,1)$-forms under this correspondence.

The first consequence of this is that $\left.\mathcal{D} \subset \operatorname{ker} \mathfrak{B}\right|_{\mathcal{H}}$; hence $\mathcal{E}=\mathcal{D} \oplus \mathcal{L}_{\text {bd }}$ by (4.2), because wedging a scalar form with $\omega$ preserves harmonicity, so that $\operatorname{tr} h$ is constant for all $h \in \mathcal{D}$, and $\operatorname{div} h=0$ because bounded harmonic forms are $d^{*}$-closed.

As a second consequence, $\operatorname{dim} \mathcal{D}=(1+2) \cdot 8+10=34$. More precisely, we saw in Section 4.1 that the space of $\Delta_{d}$-harmonic ED real $(1,1)$-forms is 8-dimensional, and that the space of all bounded $\Delta_{d}$-harmonic 2-forms modulo ED is isomorphic to the space of constant 2 -forms on the flat cylinder $\mathbb{C}^{2} / \Lambda$ to which $(M, g)$ is asymptotic; now Step 1 tells us that $\mathbb{C}^{2} / \Lambda$ has a 10-dimensional deformation space.

Remark 4.5. (i) If $I \in \mathfrak{I}\left(\mathcal{D}^{-}\right)$, then $\Delta_{\bar{\partial}} I=0$ if and only if $\bar{\partial} I=\bar{\partial}^{*} I=0$. Thus, $\mathcal{D}^{-}$ can be viewed as a space of infinitesimal deformations of the complex structure, $J$, and all of these are integrable according to Corollary 4.3 .

(ii) We can interpret the various subspaces of $\mathcal{D}$ quite concretely for the metrics coming from Theorem 1.3. As for the ED part, we have 8 Kähler directions $\perp\left[\omega_{\mathrm{CY}}\right]$; also, as per Section 3.1, the space of pencils of cubics is complex 8-dimensional. On the cylinder, we have an overall scale, the moduli $\tau, \ell^{2} / \varepsilon$ from Step 1(ii), two more real parameters that break the $S^{1} \times T^{2}$ splitting (with $J$ fixed), and the space $\mathcal{L}_{\text {lin }}$. We will see in Step 3 that the elements of $\mathcal{L}_{\text {lin }}$ lift to Lie derivatives of $g$ on $M$. 
Step 3: Lie derivatives in the kernel of the Lichnerowicz Laplacian. Our goal here is to prove that every element of the space $\mathcal{L}_{\text {lin }}$ from Step 1(i) is the constant part of a unique element $h=L_{X} g \in \mathcal{D}$, and that these are in fact all the Lie derivatives in $\mathcal{D}$. This will be a fairly direct consequence of the following technical claim.

Claim. Assume our ALH coordinate system is such that $t$ is a Busemann function. If $X$ is a vector field with $L_{X} g=$ parallel $+\mathrm{ED}$, then $|X| \leq C t$ and $\left|\nabla_{\partial_{t}} X\right| \leq C$.

Proof of the claim. For any vector field $Y$,

$$
\left\langle\nabla_{\partial_{t}} X, Y\right\rangle=\left(L_{X} g\right)\left(\partial_{t}, Y\right)-Y\left\langle X, \partial_{t}\right\rangle+\left\langle X, \nabla_{Y} \partial_{t}\right\rangle
$$

If $Y$ commutes with $\partial_{t}$, then, using the Busemann condition $\nabla_{\partial_{t}} \partial_{t}=0$,

$$
\partial_{t} Y\left\langle X, \partial_{t}\right\rangle=Y \partial_{t}\left\langle X, \partial_{t}\right\rangle=Y \frac{1}{2}\left(L_{X} g\right)\left(\partial_{t}, \partial_{t}\right)
$$

Thus, if $L_{X} g=$ parallel $+\mathrm{ED}$, then $Y\left\langle X, \partial_{t}\right\rangle=\phi+\mathrm{ED}$ with $\partial_{t} \phi=0$, and therefore $\nabla_{\partial_{t}} X+\mathrm{ED} * X \in L^{\infty}$. The claim follows from this by ODE comparison.

If $h=L_{X} g \in \mathcal{D}$, then $\nabla^{*} \nabla X=-\mathfrak{B} h=0$ by Step 2. On the other hand, $X$ has linear growth by the claim, so that $X=X_{0}+$ parallel + ED by Proposition 4.1(ii), where $X_{0}$ is a linear vector field on $\mathbb{C}^{2} / \Lambda$ (more precisely, we have $\int\left\langle X, \nabla^{*} \nabla Y\right\rangle=0$ for all ED vector fields $Y$ by the claim, so $X$ belongs to the 4-dimensional cokernel identified in Proposition 4.1(ii)). Thus, $h=h_{0}+$ ED, where $h_{0} \in \mathcal{L}_{\text {lin }}$.

Conversely, every linear vector field $X_{0}$ on $\mathbb{C}^{2} / \Lambda$ defines a unique linear growth harmonic vector field $X$ on $M$, and then indeed $h=L_{X} g \in \mathcal{D}$ because we trivially have $\mathfrak{E} h=0$, and $\mathfrak{B} h=-\nabla^{*} \nabla X=0$ by the Bochner formula from Step 2.

Remark 4.6. In [35, the issue of Lie derivatives in ker $\Delta_{\text {Lich }}$ and the possibility of breaking the $S^{1} \times T^{2}$ splitting were not considered. Moreover, $h^{1}\left(X, T_{X}\right)=8$ and $h^{1}\left(X, T_{X}(\log D)\right)=9$. On the other hand, to compare our moduli count with the results of [4] after crossing with a flat $T^{3}$, it is important to note that the relevant 39-dimensional moduli space $\mathcal{M}_{+}$in 47 parametrizes $G_{2^{-}}$forms as opposed to $G_{2^{-}}$ metrics; this results in an $\mathbb{R} P^{3}$ ambiguity. Also, the definition of $\mathcal{M}_{+}$involves fixed ALH coordinates, so that the diffeomorphisms from $\mathcal{L}_{\text {lin }}$ do not act on $\mathcal{M}_{+}$.

Remark 4.7. A folklore result says that ALH spaces can be glued by their ends to construct hyper-Kähler metrics on $K 3$; an analogous problem for $G_{2}$-manifolds was solved in 34. The appropriate dimension count is $2 \cdot 24+5+(3+1)=57$. More specifically, the $24 \mathrm{ED}$ moduli on either side pair up independently, the ones that are not ED can only be combined with their images under the gluing map, and we must include the $3+1$ kernel elements of the form $h=L_{X} g$ because the two copies of $X$ do not glue up (e.g., $X \approx t \partial_{t}$ corresponds to changing the neck length). This is interesting to compare to [48], where the $L_{X} g$ type kernel elements (which were not included in $\mathcal{M}_{+}$) are added back in by hand as gluing parameters.

\section{ACKNOWLEDGMENTS}

This paper forms part of my Ph.D. thesis 22]. I am deeply grateful to my advisor, Gang Tian, for introducing me to these issues and for his guidance. I thank Richard Bamler, Ronan Conlon, Aaron Naber, Johannes Nordström, and Yikuan Yan for very helpful discussions, John Lott for conversations leading to Problem 1.11, and the BICMR at Peking University for support during completion of a first draft of this paper in Spring 2009. Thanks to the referee for many constructive comments, 
in particular for greatly simplifying the proof of Claim 1 in the proof of Proposition 3.7 as well as Step 3 of the proof of Theorem 1.10 .

\section{REFERENCES}

1. M. Anderson, The $L^{2}$-structure of moduli spaces of Einstein metrics on 4-manifolds, Geom. Funct. Anal. 2 (1992), 29-89. MR.1143663 (92m:58017)

2. M. Atiyah, N. Hitchin, The geometry and dynamics of magnetic monopoles, M. B. Porter Lectures, Princeton University Press, Princeton, NJ, 1988. MR934202 (89k:53067)

3. W. Barth, C. Peters, A. van de Ven, Compact complex surfaces, Ergebnisse der Mathematik und ihrer Grenzgebiete (3) 4, Springer-Verlag, Berlin, 1984. MR749574 (86c:32026)

4. A. Besse, Einstein manifolds, Ergebnisse der Mathematik und ihrer Grenzgebiete (3) 10, Springer-Verlag, Berlin, 1987. MR867684 (88f:53087)

5. O. Biquard, V. Minerbe, A Kummer construction for gravitational instantons, Comm. Math. Phys., to appear.

6. P. Buser, A note on the isoperimetric constant, Ann. Sci. École Norm. Sup. (4) 15 (1982), 213-230. MR683635 (84e:58076)

7. J. Cheeger, Degeneration of Einstein metrics and metrics with special holonomy, Surveys in Differential Geometry VIII (Boston, MA, 2002), 29-73, International Press, Somerville, MA, 2003. MR2039985 (2005e:53060)

8. J. Cheeger, T. Colding, Lower bounds on Ricci curvature and the almost rigidity of warped products, Ann. of Math. (2) 144 (1996), 189-237. MR1405949 (97h:53038)

9. J. Cheeger, M. Gromov, M. Taylor, Finite propagation speed, kernel estimates for functions of the Laplace operator, and the geometry of complete Riemannian manifolds, J. Differ. Geom. 17 (1982), 15-53. MR658471 (84b:58109)

10. J. Cheeger, G. Tian, Curvature and injectivity radius estimates for Einstein 4-manifolds, J. Amer. Math. Soc. 19 (2006), 487-525. MR2188134 (2006i:53042)

11. S. Cherkis, N. Hitchin, Gravitational instantons of type $D_{k}$, Comm. Math. Phys. 260 (2005), 299-317. MR2177322(2007a:53095)

12. S. Cherkis, A. Kapustin, Hyper-Kähler metrics from periodic monopoles, Phys. Rev. D (3) 65 (2002), 084015. MR:1899201 (2003g:53069)

13. S. Donaldson, Calabi-Yau metrics on Kummer surfaces as a model gluing problem, preprint, arXiv:1007.4218.

14. D. Freed, Special Kähler manifolds, Comm. Math. Phys. 203 (1999), 31-52. MR.1695113 (2000f:53060)

15. R. Gompf, A. Stipsicz, 4-manifolds and Kirby calculus, Graduate Studies in Mathematics 20, American Mathematical Society, Providence, RI, 1999. MR,1707327 (2000h:57038)

16. R. Goto, Moduli spaces of topological calibrations, Calabi-Yau, hyper-Kähler, $G_{2}$ and Spin(7) structures, Internat. J. Math. 15 (2004), 211-257. MR2060789(2005f:53082)

17. B. Greene, A. Shapere, C. Vafa, S.-T. Yau, Stringy cosmic strings and noncompact Calabi-Yau manifolds, Nuclear Phys. B 337 (1990), 1-36. MR.1059826 (91g:83057)

18. P. Griffiths, J. Harris, Principles of algebraic geometry, Pure and Applied Mathematics, Wiley-Interscience, New York, 1978. MR507725 (80b:14001)

19. A. Grigoryan, L. Saloff-Coste, Stability results for Harnack inequalities, Ann. Inst. Fourier 55 (2005), 825-890. MR2149405 (2006b:58026)

20. M. Gross, P. Wilson, Large complex structure limits of K3 surfaces, J. Differ. Geom. 55 (2000), 475-546. MR1863732(2003a:32042)

21. B. Harbourne, W. Lang, Multiple fibers on rational elliptic surfaces, Trans. Amer. Math. Soc. 307 (1988), 205-223. MR936813 (89j:14024)

22. H.-J. Hein, On gravitational instantons, Ph.D. thesis, Princeton University, September 2010. MR2813955

23. H.-J. Hein, Weighted Sobolev inequalities under lower Ricci curvature bounds, Proc. Amer. Math. Soc. 139 (2011), 2943-2955. MR2801635

24. N. Hitchin, Twistor construction of Einstein metrics, Global Riemannian geometry (Durham, 1983), 115-125, Ellis Horwood Ser.: Math. Appl., Horwood, Chichester, 1984. MR757213 (85m:53048)

25. N. Hitchin, The moduli space of complex Lagrangian submanifolds, Asian J. Math. 3 (1999), 77-92. MR.1701923 (2000m:32023) 
26. D. Huybrechts, Complex geometry, Universitext, Springer-Verlag, Berlin, 2005. MR2093043 (2005h:32052)

27. D. Joyce, Asymptotically locally Euclidean metrics with holonomy SU $(m)$, Ann. Global Anal. Geom. 19 (2001), 55-73. MR1824171(2002i:53063)

28. F. Klein, Vorlesungen über die Theorie der elliptischen Modulfunctionen, Erster Band, Teubner, Leipzig, 1890.

29. K. Kodaira, On compact analytic surfaces, II, Ann. of Math. (2) 77 (1963), 563-626. MR0184257(32:1730)

30. K. Kodaira, L. Nirenberg, D. Spencer, On the existence of deformations of complex analytic structures, Ann. of Math. (2) 68 (1958), 450-459. MR0112157 (22:3012)

31. K. Kodaira, D. Spencer, On deformations of complex analytic structures, III, Stability theorems for complex structures, Ann. of Math. (2) 71 (1960), 43-76. MR0115189 (22:5991)

32. B. Köhler, M. Kühnel, On asymptotics of complete Ricci-flat Kähler metrics on open manifolds, Manuscripta Math. 132 (2010), 431-462. MR2652441(2011h:32025)

33. N. Koiso, Einstein metrics and complex structures, Invent. Math. 73 (1983), 71-106. MR707349 (85d:58018)

34. A. Kovalev, Twisted connected sums and special Riemannian holonomy, J. reine angew. Math. 565 (2003), 125-160. MR2024648(2004m:53088)

35. A. Kovalev, Ricci-flat deformations of asymptotically cylindrical Calabi-Yau manifolds, Proceedings of Gökova Geometry-Topology Conference 2005, 140-156, Gökova, 2006. MR2282013 (2008c:32034)

36. P. Kronheimer, The construction of ALE spaces as hyper-Kähler quotients, J. Differ. Geom. 29 (1989), 665-683. MR992334 (90d:53055)

37. P. Kronheimer, A Torelli-type theorem for gravitational instantons, J. Differ. Geom. 29 (1989), 685-697. MR992335 (90d:53056)

38. C. LeBrun, Complete Ricci-flat Kähler metrics on $\mathbb{C}^{n}$ need not be flat, Several complex variables and complex geometry (Santa Cruz, CA, 1989), 297-304, Proc. Sympos. Pure Math. 52, Part 2, American Mathematical Society, Providence, RI, 1991. MR1128554 (93a:53038)

39. P. Li, L.-F. Tam, Green's functions, harmonic functions, and volume comparison, J. Differ. Geom. 41 (1995), 277-318. MR1331970 (96f:53054)

40. J. Loftin, Singular semi-flat Calabi-Yau metrics on $S^{2}$, Comm. Anal. Geom. 13 (2005), 333361. MR2154822 (2006d:32035)

41. P. Maheux, L. Saloff-Coste, Analyse sur les boules d'un opérateur sous-elliptique, Math. Ann. 303 (1995), 713-740. MR 1359957 (96m:35049)

42. V. Minerbe, On the asymptotic geometry of gravitational instantons, Ann. Sci. École Norm. Sup. (4) 43 (2010), 883-924. MR2778451

43. V. Minerbe, Rigidity for Multi-Taub-NUT metrics, J. reine angew. Math. 656 (2011), 47-58.

44. R. Miranda, The moduli of Weierstrass fibrations over $\mathbf{P}^{1}$, Math. Ann. 255 (1981), 379-394. MR615858 (83b:14010)

45. R. Miranda, Persson's list of singular fibers for a rational elliptic surface, Math. Z. 205 (1990), 191-211. MR1076128 (92a:14035)

46. A. Naber, G. Tian, Geometric structures of collapsing Riemannian manifolds, I, preprint, arXiv:0804.2275.

47. J. Nordström, Deformations of asymptotically cylindrical $G_{2}$-manifolds, Math. Proc. Camb. Phil. Soc. 145 (2008), 311-348. MR2442130(2009e:53068)

48. J. Nordström, Deformations of glued $G_{2}$-manifolds, Comm. Anal. Geom. 17 (2009), 481-503. MR2550206 (2011b:53118)

49. B. Santoro, Existence of complete Kähler Ricci-flat metrics on crepant resolutions, preprint, arXiv:0902.0595.

50. J. Song, G. Tian, The Kähler-Ricci flow on surfaces of positive Kodaira dimension, Invent. Math. 170 (2007), 609-653. MR2357504 (2008m:32044)

51. G. Tian, Smoothness of the universal deformation space of Calabi-Yau manifolds and its Petersson-Weil metric, Mathematical aspects of string theory (San Diego, CA, 1986), 629646, Adv. Ser. Math. Phys. 1, World Sci. Publishing, Singapore, 1987. MR915841

52. G. Tian, Aspects of metric geometry of four manifolds, Inspired by S. S. Chern, 381-397, Nankai Tracts Math. 11, World Scientific Publishing, Hackensack, NJ, 2006. MR2313343 (2008i:53044) 
53. G. Tian, S.-T. Yau, Complete Kähler manifolds with zero Ricci curvature, I, J. Amer. Math. Soc. 3 (1990), 579-609. MR1040196 (91a:53096)

54. G. Tian, S.-T. Yau, Complete Kähler manifolds with zero Ricci curvature, II, Invent. Math. 106 (1991), 27-60. MR1123371 (92j:32028)

55. A. Todorov, The Weil-Petersson geometry of the moduli space of $\mathrm{SU}(n \geq 3)$ (Calabi-Yau) manifolds, I, Comm. Math. Phys. 126 (1989), 325-346. MR.1027500 (91f:32022)

56. V. Tosatti, Adiabatic limits of Ricci-flat Kähler metrics, J. Differ. Geom. 84 (2010), 427-453. MR2652468(2011m:32039)

Department of Mathematics, Imperial College, London SW7 2AZ, United Kingdom

E-mail address: h.hein@imperial.ac.uk 